\title{
SLUDGE BATCH 5 VARIABILITY STUDY WITH FRIT 418
}

\author{
F.C. Raszewski \\ T.B. Edwards \\ D.K. Peeler
}

September 2008

Environmental \& Chemical Process Technology Savannah River National Laboratory Aiken, SC 29808

Prepared for the U.S. Department of Energy Under Contract Number DE-AC09-08SR22470

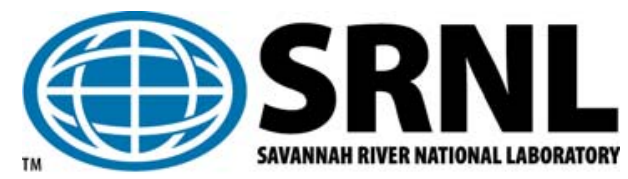


SRNS-STI-2008-00065

Revision 0

\section{DISCLAIMER}

This work was prepared under an agreement with and funded by the U.S. Government. Neither the U.S. Government or its employees, nor any of its contractors, subcontractors or their employees, makes any express or implied: 1. warranty or assumes any legal liability for the accuracy, completeness, or for the use or results of such use of any information, product, or process disclosed; or 2. representation that such use or results of such use would not infringe privately owned rights; or 3. endorsement or recommendation of any specifically identified commercial product, process, or service. Any views and opinions of authors expressed in this work do not necessarily state or reflect those of the United States Government, or its contractors, or subcontractors.

This document was prepared in conjunction with work accomplished under Contract No. DE-AC09-08SR22470 with the U.S. Department of Energy. 
Keywords: SB5, Frit 418, variability, durability

Retention: permanent

\title{
SLUDGE BATCH 5 VARIABILITY STUDY WITH FRIT 418
}

\author{
F.C. Raszewski \\ T.B. Edwards \\ D.K. Peeler
}

September 2008

Environmental \& Chemical Process Technology Savannah River National Laboratory Aiken, SC 29808

Prepared for the U.S. Department of Energy Under Contract Number DE-AC09-08SR22470

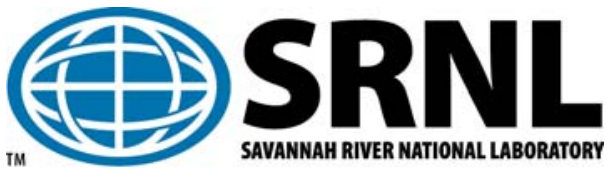




\section{REVIEWS AND APPROVALS}

\section{AUTHORS:}

F.C. Raszewski, Process Engineering Technology

Date

T.B. Edwards, Statistical Consulting Section

Date

D.K. Peeler, Process Engineering Technology

Date

\section{TECHNICAL REVIEWER:}

K.M. Fox, Process Engineering Technology

Date

APPROVERS:

J.C. Griffin, Manager, E\&CPT Research Programs

Date

C.C. Herman, Manager, Process Engineering Technology

Date

J.E. Occhipinti, Manager, Waste Solidification Engineering

Date 


\section{EXECUTIVE SUMMARY}

The Defense Waste Processing Facility (DWPF) is preparing to initiate processing Sludge Batch 5 (SB5) in early FY 2009. In support of the upcoming processing, the Savannah River National Laboratory (SRNL) provided a recommendation to utilize Frit 418 as a transitional frit to initiate processing of SB5. This recommendation was based on the results of assessments on the compositional projections for SB5 available at that time from both the Liquid Waste Organization (LWO) and SRNL (using a model-based approach). To support qualification of the Frit 418-SB5 system, SRNL executed a variability study to assess the acceptability of the Frit 418-SB5 glasses with respect to durability and the applicability of the current durability models.

Twenty one glasses were selected for the variability study based on the available SB5 projections primarily spanning a waste loading (WL) range of $25-37 \%$. In order to account for the addition of caustic to Tank 40, which occurred in July 2008, $3 \mathrm{wt} \% \mathrm{Na}_{2} \mathrm{O}$ was added to the original Tank 40 heel projections. The addition of the Actinide Removal Process (ARP) stream to the blend composition was also included. Two of the glasses were fabricated at $25 \%$ and $28 \%$ WL in order to challenge the homogeneity constraint of the Product Composition Control System (PCCS) for SB5 coupled operations. These twenty one glasses were fabricated and characterized using chemical composition analysis, X-ray Diffraction (XRD) and the Product Consistency Test (PCT).

The results of this study indicate that Frit 418 is a viable option for sludge-only and coupled operations. The addition of ARP did not have any negative impacts on the acceptability and predictability of the variability study glasses.

Based on the measured PCT response, all of the glasses were acceptable as compared to the Environmental Assessment (EA) reference glass regardless of the thermal history and were also predictable using the current PCCS model for durability.

The homogeneity constraint can be ignored for SB5 for coupled operations (based on the Appendix J compositional information), as both of the glasses targeting lower WLs (SB5VS-18 and SB5VS-19) had durabilities that were acceptable with respect to the reference EA glass. 


\section{TABLE OF CONTENTS}

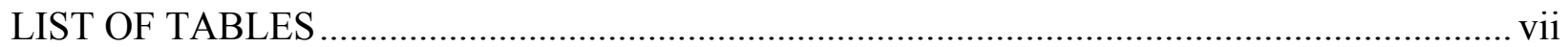

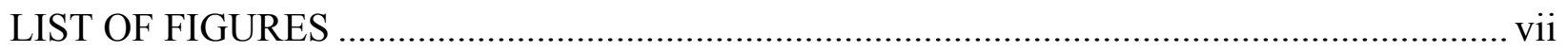

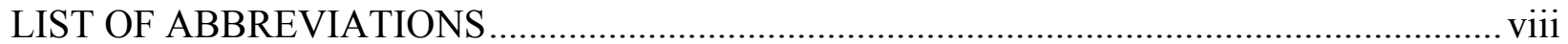

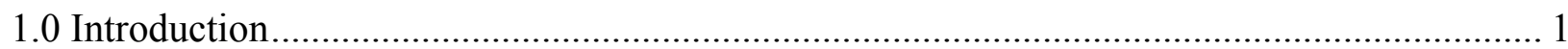

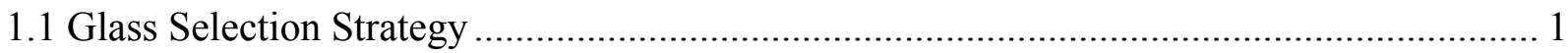

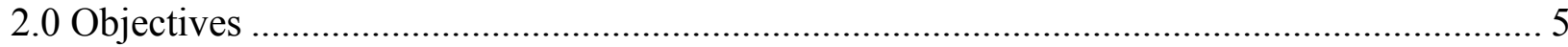

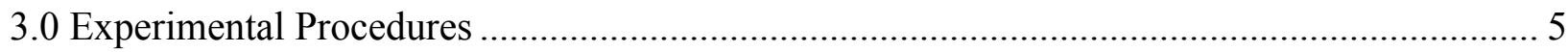

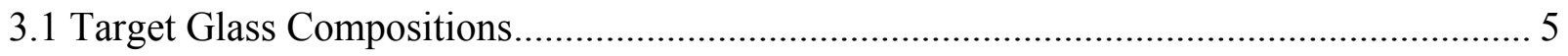

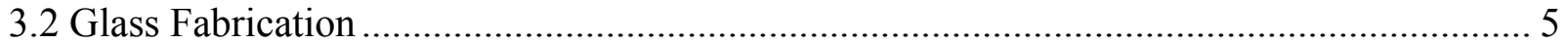

3.3 Property Measurements .............................................................................................. 5

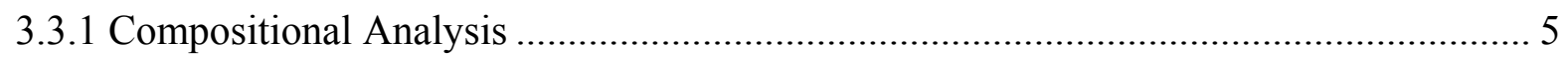

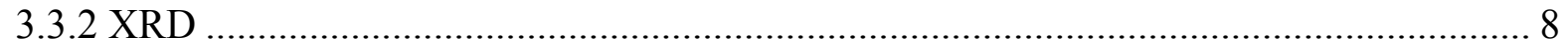

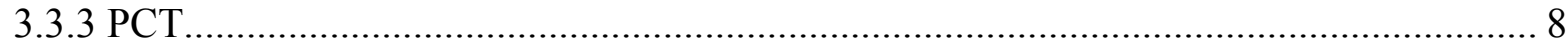

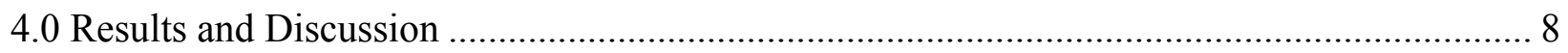

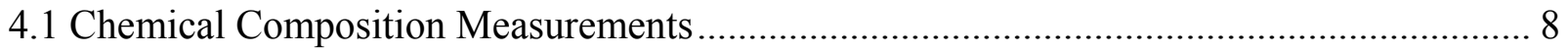

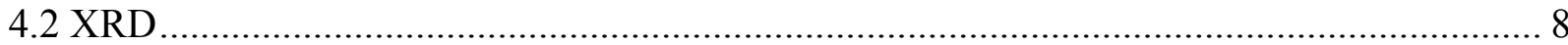

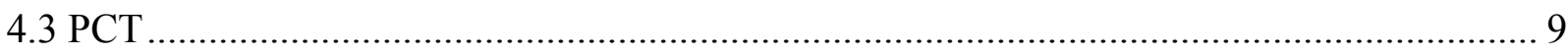

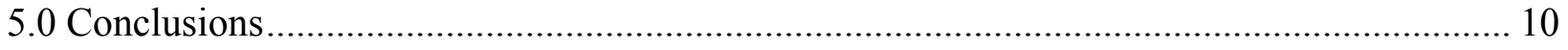

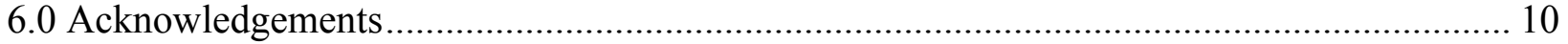

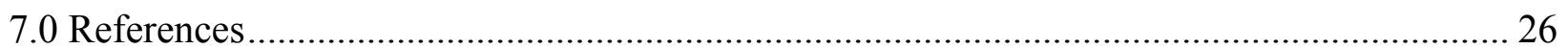




\section{LIST OF TABLES}

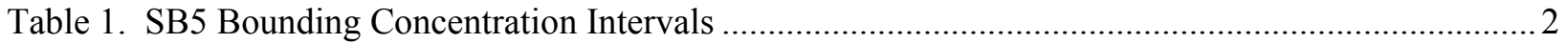

Table 2. Twelve Bounding SB5 Sludge Compositions and the Centroid ................................................. 3

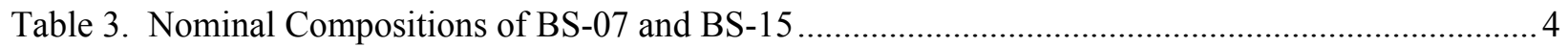

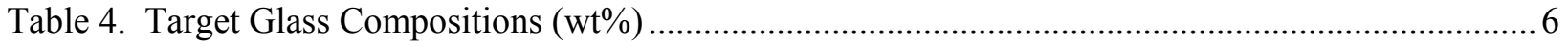

Table 5. Summary of the Target, Average Measured/Bias-Corrected Chemical Compositions ............... 11

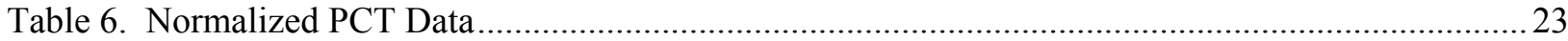

\section{LIST OF FIGURES}

Figure 1. A representative XRD pattern for an amorphous quenched (a) and ccc (b) sample..................9

Figure 2. $\log N L[B(g / L)]$ versus del $G_{p}\left(\Delta G_{p}\right)$ model with a 95\% confidence interval for individual PCTs. 


\section{LIST OF ABBREVIATIONS}

\begin{tabular}{|c|c|}
\hline $\mathrm{AD}$ & Analytical Development \\
\hline ARM & Approved Reference Material \\
\hline ARP & Actinide Removal Process \\
\hline $\mathrm{bc}$ & Bias Correction \\
\hline B Del $G_{p}$ & $\Delta \mathrm{G}_{\mathrm{p}}$ value for boron \\
\hline $\mathrm{ccc}$ & Centerline Canister Cooling \\
\hline DWPF & Defense Waste Processing Facility \\
\hline EA & Environmental Assessment \\
\hline ICP-AES & Inductively Coupled Plasma-Atomic Emission Spectroscopy \\
\hline ID & Identification \\
\hline LM & Lithium Metaborate \\
\hline LWO & Liquid Waste Organization \\
\hline MAR & Measure Acceptability Region \\
\hline $\mathrm{NL}[\mathrm{B}]$ & Normalized Leachate for Boron \\
\hline NL[Li] & Normalized Leachate for Lithium \\
\hline $\mathrm{NL}[\mathrm{Na}]$ & Normalized Leachate for Sodium \\
\hline $\mathrm{NL}[\mathrm{Si}]$ & Normalized Leachate for Silicon \\
\hline PCCS & Product Composition Control System \\
\hline PCT & Product Consistency Test \\
\hline $\mathrm{PF}$ & Sodium Peroxide Fusion \\
\hline PSAL & Process Science Analytical Laboratory \\
\hline SB5 & Sludge Batch 5 \\
\hline SME & Slurry Mix Evaporator \\
\hline SRAT & Sludge Receipt and Adjustment Tank \\
\hline SRNL & Savannah River National Laboratory \\
\hline $\mathrm{T}_{\mathrm{L}}$ Pred & Liquidus Temperature Prediction \\
\hline $\mathrm{U}_{\text {std }}$ & Uranium Standard \\
\hline Visc Pred & Viscosity Prediction \\
\hline WL & Waste Loading \\
\hline XRD & X-ray Diffraction \\
\hline
\end{tabular}




\subsection{Introduction}

The Defense Waste Processing Facility (DWPF) is preparing to initiate processing Sludge Batch 5 (SB5) in early FY 2009. In support of the upcoming processing, the Savannah River National Laboratory (SRNL) provided a recommendation to utilize Frit $418^{\mathrm{a}}$ as a transitional frit to initiate processing of SB5. ${ }^{1}$ This recommendation was based on the results of assessments on the compositional projections for SB5 available at that time from both the Liquid Waste Organization (LWO) ${ }^{\mathrm{b}}$ and SRNL (using a model-based approach). ${ }^{\mathrm{c}, \mathrm{d}}$ It should be noted that further experimental testing is required to optimize a frit composition for melt rate as Frit 418 was primarily selected due to its robustness to compositional uncertainty of the blending and/or washing strategy for SB5.

To support qualification of the Frit 418-SB5 system, SRNL executed a variability study to assess the acceptability of the Frit 418-SB5 glasses with respect to durability and the applicability of the current durability models. The experimental portion of the variability study was initiated by a DWPF Technical Task Request (TTR) ${ }^{2}$ and was performed under a SRNL Task Technical and Quality Assurance Plan (TT\&QAP). ${ }^{3}$

\subsection{Glass Selection Strategy}

For a complete discussion of the sludge projections see WSRC-STI-2008-00338. ${ }^{1}$ Only a brief summary of those specific projections used to the support the variability study will be provided in this section.

The projections for the composition of SB5 documented in WSRC-STI-2008-00338 cover the various SB5 projections from LWO and SRNL modeling efforts. ${ }^{1}$ These compositions were utilized in two ways for input to this variability study. The first approach utilized the sludge region bounded by the variation stage assessment discussed in WSRC-STI-2008-00338. This approach was used to select sludge compositions from the bounding region of SB5 compositional possibilities to demonstrate the robustness of the SB5-Frit 418 glass system to sludge variation. Specifically, the bounding intervals for sludge components developed in that report (presented in the report as Table 4-1) are repeated as Table 1 below. The "Others" component is used to represent a group of minor oxides that are handled collectively in the projected composition of SB5 (see WSRC-STI-2008-00338 for details). The intervals of Table 1 provided the basis for selecting 12 bounding compositions for SB5. The 12 sludge compositions were selected by employing the Coordinate Exchange algorithm that is available in the Custom Design platform of $\mathrm{JMP}^{\mathrm{TM}}$ Version 6.0.3. ${ }^{4}$ Twelve is the minimum number of design points that could be selected from the region defined by Table 1 since the table defines 12 components (including "Others") that are required to fully identify a sludge composition. The SB5 centroid composition (average of the bounding sludge compositions) was also utilized in the variability study. The 12 bounding compositions and the SB5 centroid were combined with Frit 418 at a $34 \%$ WL (Table 2). ${ }^{f}$

\footnotetext{
${ }^{\text {a }} 8 \mathrm{~B}_{2} \mathrm{O}_{3}-8 \mathrm{Li}_{2} \mathrm{O}-8 \mathrm{Na}_{2} \mathrm{O}-76 \mathrm{SiO}_{2}(\mathrm{wt} \%)$

${ }^{\mathrm{b}}$ The most recent SB5 composition projections (those used in this study) were received from LWO via email from D. D. Larsen on June 5, 2008. Washing data used to calculate a $\mathrm{SO}_{4}{ }^{2-}$ concentration were included in a spreadsheet attached to the email, titled 'SB4-5_060408_For Variability Study Comparison.xls.xlsm'. See WSRC-NB-2007-00003 for further detail.

${ }^{\mathrm{c}}$ The composition projections developed by SRNL and used in this study were received via email from A. S. Choi on June 12 , 2008. See WSRC-NB-2007-00003 for further detail.

${ }^{\mathrm{d}}$ For further information on the SRNL model-based approach, see Choi, A. S., "Aluminum Dissolution Flowsheet Modeling in Support of SB5 Frit Development," Savannah River National Laboratory, Aiken, SC, Report No. WSRC-STI-2008-00001, Rev. $0,2008$.

${ }^{\mathrm{e}}$ Based on recent melt rate information, Frit $550\left(12 \mathrm{~B}_{2} \mathrm{O}_{3}-8 \mathrm{Li}_{2} \mathrm{O}-7 \mathrm{Na}_{2} \mathrm{O}-73 \mathrm{SiO}_{2}[\mathrm{wt} \%]\right)$ has been identified as a primary candidate for potentially increasing the melt rate of SB5 (relative to the Frit 418 based system). Slurry fed melt rate testing is planned in the October-November 2008 timeframe with both Frit 418 and Frit 550. If DWPF elects to utilize Frit 550 based on the melt rate data, a supplemental variability study would be required.

${ }^{\mathrm{f}} 34 \% \mathrm{WL}$ is the nominal target for SB5 processing.
} 
SRNS-STI-2008-00065

Revision 0

Table 1. SB5 Bounding Concentration Intervals

\begin{tabular}{|c|c|c|}
\hline Oxide & $\begin{array}{c}\text { Minimum } \\
\text { (wt\%) }\end{array}$ & $\begin{array}{c}\text { Maximum } \\
\text { (wt\%) }\end{array}$ \\
\hline $\mathrm{Al}_{2} \mathrm{O}_{3}$ & 18.977 & 24.211 \\
\hline $\mathrm{CaO}$ & 1.840 & 3.119 \\
\hline $\mathrm{Fe}_{2} \mathrm{O}_{3}$ & 25.292 & 32.814 \\
\hline $\mathrm{MgO}$ & 1.024 & 2.250 \\
\hline $\mathrm{MnO}$ & 5.299 & 6.877 \\
\hline $\mathrm{Na}_{2} \mathrm{O}$ & 19.332 & 29.245 \\
\hline $\mathrm{NiO}$ & 2.377 & 3.820 \\
\hline $\mathrm{SO}_{4}$ & 0.310 & 0.857 \\
\hline $\mathrm{SiO}_{2}$ & 1.649 & 3.056 \\
\hline $\mathrm{TiO}_{2}$ & 0.000 & 1.815 \\
\hline $\mathrm{U}_{3} \mathrm{O}_{8}$ & 7.302 & 9.505 \\
\hline $\mathrm{Others}_{2}$ & 0.284 & 1.284 \\
\hline
\end{tabular}

Two of the nominal compositions from WSRC-STI-2008-00338 (BS-07 [3 wt $\% \mathrm{Na}_{2} \mathrm{O}$ addition to the Tank 40 heel] and BS-15 [3 wt $\% \mathrm{Na}_{2} \mathrm{O}$ addition to the Tank 40 heel and the addition of the Actinide Removal Process (ARP) stream] $)^{g}$ were combined with Frit 418 at various WLs to complete the selection of glasses for this variability study (Table 3). ${ }^{\mathrm{h}}$ These two SB5 projections were based only on SRNL projections, as they more closely represented the composition of the SB5 qualification sample. ${ }^{5}$ Each of these nominal sludge compositions was combined with Frit 418 at WLs of 31\%, 34\%, and 37\% to demonstrate the operating windows for these glass systems. In addition, the BS-15 projection was combined with Frit 418 at WLs of $25 \%$ and $28 \%$ to intentionally challenge the homogeneity constraint for this SB5 projection as a coupled-operations flowsheet. ${ }^{6}$

A total of twenty one glasses were fabricated and characterized using chemical composition analysis, Xray Diffraction (XRD) and the Product Consistency Test (PCT).

\footnotetext{
${ }^{\mathrm{g}}$ BS-07 and BS-15 are based on the SB5 blend projection, which is composed of a mass ratio of 25:75 (Tank 40 heel to the Tank 51 SB5 batch). Compositional information for ARP additions was obtained from Appendix J of S.G. Subosits, "Actinide Removal Process Material Balance Calculation with Low Curie Salt Feed," X-CLC-S-00113, Rev. 0, September 24, 2004.

${ }^{\mathrm{h}}$ See the Measurement Acceptability Region (MAR) results for the nominal stage assessment provided in WSRC-STI-200800338 for details of the projected operating windows for the BS-07 and BS-15 options with Frit 418.
} 
Table 2. Twelve Bounding SB5 Sludge Compositions and the Centroid

\begin{tabular}{|c|c|c|c|c|c|c|c|c|c|c|c|c|}
\hline Type & $\mathrm{Al}_{2} \mathrm{O}_{3}$ & $\mathrm{CaO}$ & $\mathrm{Fe}_{2} \mathrm{O}_{3}$ & $\mathrm{MgO}$ & $\mathrm{MnO}$ & $\mathrm{Na}_{2} \mathrm{O}$ & $\mathrm{NiO}$ & $\mathrm{SO}_{4}$ & $\mathrm{SiO}_{2}$ & $\mathrm{TiO}_{2}$ & $\mathrm{U}_{3} \mathrm{O}_{8}$ & Others \\
\hline Bounding & 18.977 & 1.84 & 32.4 & 1.024 & 6.572 & 23.63 & 2.377 & 0.857 & 2.924 & 1.813 & 7.302 & 0.284 \\
\hline Bounding & 24.085 & 1.84 & 25.292 & 2.223 & 5.299 & 25.682 & 3.674 & 0.857 & 1.649 & 1.813 & 7.302 & 0.284 \\
\hline Bounding & 18.977 & 3.016 & 32.4 & 2.223 & 5.299 & 19.922 & 3.674 & 0.38 & 2.924 & 1.813 & 9.088 & 0.284 \\
\hline Bounding & 18.977 & 3.016 & 25.292 & 1.024 & 6.572 & 29.016 & 3.674 & 0.38 & 1.649 & 1.813 & 7.302 & 1.284 \\
\hline Bounding & 24.085 & 3.016 & 32.33 & 2.223 & 6.572 & 19.781 & 2.377 & 0.38 & 1.649 & 0 & 7.302 & 0.284 \\
\hline Bounding & 24.085 & 3.016 & 25.292 & 1.024 & 6.572 & 23.184 & 3.674 & 0.857 & 2.924 & 0 & 9.088 & 0.284 \\
\hline Bounding & 18.977 & 3.016 & 26.496 & 2.223 & 5.299 & 29.245 & 2.377 & 0.857 & 2.924 & 0 & 7.302 & 1.284 \\
\hline Bounding & 24.085 & 1.84 & 32.4 & 1.024 & 5.299 & 19.788 & 3.674 & 0.38 & 2.924 & 0 & 7.302 & 1.284 \\
\hline Bounding & 24.085 & 1.84 & 25.292 & 2.223 & 6.572 & 22.122 & 2.377 & 0.38 & 2.924 & 1.813 & 9.088 & 1.284 \\
\hline Bounding & 24.085 & 3.016 & 29.726 & 1.024 & 5.299 & 19.781 & 2.377 & 0.857 & 1.649 & 1.813 & 9.088 & 1.284 \\
\hline Bounding & 18.977 & 1.84 & 32.4 & 2.223 & 6.572 & 21.435 & 3.674 & 0.857 & 1.649 & 0 & 9.088 & 1.284 \\
\hline Bounding & 18.977 & 1.84 & 29.836 & 1.024 & 5.299 & 29.245 & 2.377 & 0.38 & 1.649 & 0 & 9.088 & 0.284 \\
\hline SB5 Centroid & 21.266 & 2.425 & 28.713 & 1.621 & 5.93 & 24.248 & 3.019 & 0.619 & 2.281 & 0.901 & 8.191 & 0.785 \\
\hline
\end{tabular}


Table 3. Nominal Compositions of BS-07 and BS-15

\begin{tabular}{|c|c|c|}
\hline Sludge ID & 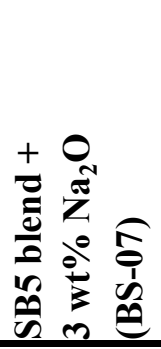 & 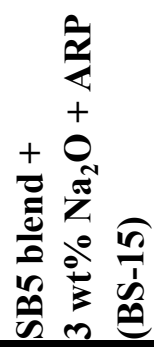 \\
\hline $\mathrm{Al}_{2} \mathrm{O}_{3}$ & 21.915 & 21.127 \\
\hline $\mathrm{BaO}$ & 0.142 & 0.139 \\
\hline $\mathrm{CaO}$ & 2.465 & 2.391 \\
\hline $\mathrm{CdO}$ & 0.098 & 0.093 \\
\hline $\mathrm{Ce}_{2} \mathrm{O}_{3}$ & 0.016 & 0.024 \\
\hline $\mathrm{Cr}_{2} \mathrm{O}_{3}$ & 0.102 & 0.102 \\
\hline $\mathrm{CuO}$ & 0.025 & 0.026 \\
\hline $\mathrm{Fe}_{2} \mathrm{O}_{3}$ & 29.632 & 28.752 \\
\hline $\mathrm{K}_{2} \mathrm{O}$ & 0.13 & 0.129 \\
\hline $\mathrm{La}_{2} \mathrm{O}_{3}$ & 0.012 & 0.015 \\
\hline $\mathrm{Li}_{2} \mathrm{O}$ & 0.013 & 0.013 \\
\hline $\mathrm{MgO}$ & 1.62 & 1.544 \\
\hline $\mathrm{MnO}$ & 6.018 & 5.943 \\
\hline $\mathrm{Na}_{2} \mathrm{O}$ & 22.753 & 23.596 \\
\hline $\mathrm{NiO}$ & 3.159 & 3.068 \\
\hline $\mathrm{P}_{2} \mathrm{O}_{5}$ & 0.332 & 0.318 \\
\hline $\mathrm{PbO}$ & 0.013 & 0.018 \\
\hline $\mathrm{SO}_{4}$ & 0.621 & 0.732 \\
\hline $\mathrm{SiO}_{2}$ & 2.227 & 2.149 \\
\hline $\mathrm{TiO}_{2}$ & 0.01 & 1.307 \\
\hline $\mathrm{U}_{3} \mathrm{O}_{8}$ & 8.329 & 8.072 \\
\hline $\mathrm{ZnO}$ & 0.01 & 0.014 \\
\hline $\mathrm{ZrO}_{2}$ & 0.148 & 0.15 \\
\hline
\end{tabular}




\subsection{Objectives}

The objective of the experimental portion of the variability study was to demonstrate that the glasses of the Frit 418-SB5 compositional region had chemical durabilities that were both acceptable relative to the Environmental Assessment (EA) reference glass and predictable by the current process control models for durability.

\subsection{Experimental Procedures}

\subsection{Target Glass Compositions}

Target glass compositions of the twenty one SB5 variability study glasses are presented in Table 4. The nomenclature for the glass identification (ID) can be described as follows: "SB5VS" refers to Sludge Batch 5 Variability Study.

\subsection{Glass Fabrication}

Each variability study glass was prepared from the proper proportions of reagent-grade metal oxides, carbonates, $\mathrm{H}_{3} \mathrm{BO}_{3}$, and salts in $150 \mathrm{~g}_{\text {batches. }}{ }^{7}$ The raw materials were thoroughly mixed and placed into a platinum alloy, $250 \mathrm{ml}$ crucible. Batched materials were placed into a high-temperature furnace at the target melt temperature of $1150^{\circ} \mathrm{C}$. The crucible was removed from the furnace after an isothermal hold at $1150^{\circ} \mathrm{C}$ for 1 hour. The molten glass was quenched by pouring the liquid onto a clean, stainless steel plate. The glass pour patty was used as a sampling stock for the various property measurements (i.e., chemical composition, durability testing and XRD).

Approximately $25 \mathrm{~g}$ of each glass was heat-treated to simulate cooling along the centerline of a DWPFtype canister to gauge the effects of thermal history on the product performance. ${ }^{8}$ This cooling schedule is referred to as the centerline canister cooling (ccc) curve.

\subsection{Property Measurements}

\subsubsection{Compositional Analysis}

To confirm that the as-fabricated glasses met the target compositions, a representative sample from each glass was submitted to Analytical Development (AD) for chemical analysis under the auspices of an analytical plan (SRNL-SCS-2008-00071). ${ }^{9}$ Two dissolution methods were utilized in measuring these chemical compositions: lithium metaborate fusion (LM) and sodium peroxide fusion (PF). For each study glass, measurements were obtained from samples prepared in duplicate by each of these dissolution methods. All of the prepared samples were analyzed (twice for each element of interest) by Inductively Coupled Plasma-Atomic Emission Spectroscopy (ICP-AES) with the instrumentation being re-calibrated between the duplicate analyses. The analytical plan was developed in such a way as to provide the opportunity to evaluate potential sources of bias and error. Glass standards were also intermittently measured to assess the performance of the ICP-AES instrument over the course of these analyses. 
Table 4. Target Glass Compositions (wt\%)

\begin{tabular}{|c|c|c|c|c|c|c|c|c|c|c|c|c|c|}
\hline \multirow{3}{*}{ Glass ID } & \multicolumn{12}{|c|}{ Bounding Compositions } & \multirow{2}{*}{ 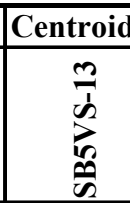 } \\
\hline & 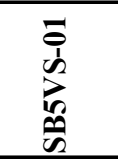 & 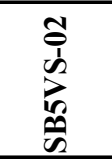 & 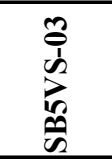 & 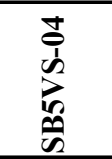 & 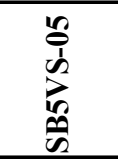 & 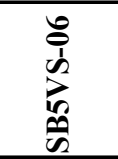 & 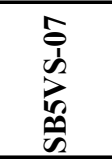 & 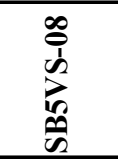 & 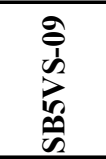 & $\begin{array}{l}\frac{1}{1} \\
\tilde{n} \\
\frac{1}{10} \\
\frac{1}{0} \\
0\end{array}$ & 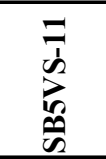 & 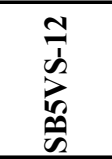 & \\
\hline & \multicolumn{13}{|c|}{$34 \% \mathrm{WL}$} \\
\hline $\mathrm{Al}_{2} \mathrm{O}_{3}$ & 6.452 & 8.189 & 6.452 & 6.452 & 8.189 & 8.189 & 6.452 & 8.189 & 8.189 & 8.189 & 6.452 & 6.452 & 7.230 \\
\hline $\mathrm{B}_{2} \mathbf{O}_{3}$ & 5.280 & 5.280 & 5.280 & 5.280 & 5.280 & 5.280 & 5.280 & 5.280 & 5.280 & 5.280 & 5.280 & 5.280 & 5.280 \\
\hline $\mathrm{BaO}$ & 0.010 & 0.010 & 0.010 & 0.045 & 0.010 & 0.010 & 0.045 & 0.045 & 0.045 & 0.045 & 0.045 & 0.010 & 0.027 \\
\hline $\mathrm{CaO}$ & 0.626 & 0.626 & 1.026 & 1.026 & 1.026 & 1.026 & 1.026 & 0.626 & 0.626 & 1.026 & 0.626 & 0.626 & 0.825 \\
\hline CdO & 0.006 & 0.006 & 0.006 & 0.029 & 0.006 & 0.006 & 0.029 & 0.029 & 0.029 & 0.029 & 0.029 & 0.006 & 0.018 \\
\hline $\mathrm{Ce}_{2} \mathrm{O}_{3}$ & 0.003 & 0.003 & 0.003 & 0.012 & 0.003 & 0.003 & 0.012 & 0.012 & 0.012 & 0.012 & 0.012 & 0.003 & 0.008 \\
\hline $\mathrm{Cr}_{2} \mathrm{O}_{3}$ & 0.009 & 0.009 & 0.009 & 0.042 & 0.009 & 0.009 & 0.042 & 0.042 & 0.042 & 0.042 & 0.042 & 0.009 & 0.026 \\
\hline $\mathrm{CuO}$ & 0.003 & 0.003 & 0.003 & 0.012 & 0.003 & 0.003 & 0.012 & 0.012 & 0.012 & 0.012 & 0.012 & 0.003 & 0.008 \\
\hline $\mathrm{Fe}_{2} \mathrm{O}_{3}$ & 11.016 & 8.599 & 11.016 & 8.599 & 10.992 & 8.599 & 9.009 & 11.016 & 8.599 & 10.107 & 11.016 & 10.144 & 9.762 \\
\hline $\mathrm{K}_{2} \mathrm{O}$ & 0.010 & 0.010 & 0.010 & 0.045 & 0.010 & 0.010 & 0.045 & 0.045 & 0.045 & 0.045 & 0.045 & 0.010 & 0.028 \\
\hline $\mathrm{La}_{2} \mathrm{O}_{3}$ & 0.002 & 0.002 & 0.002 & 0.009 & 0.002 & 0.002 & 0.009 & 0.009 & 0.009 & 0.009 & 0.009 & 0.002 & 0.005 \\
\hline $\mathbf{L i}_{2} \mathbf{O}$ & 5.281 & 5.281 & 5.281 & 5.284 & 5.281 & 5.281 & 5.284 & 5.284 & 5.284 & 5.284 & 5.284 & 5.281 & 5.282 \\
\hline MgO & 0.348 & 0.756 & 0.756 & 0.348 & 0.756 & 0.348 & 0.756 & 0.348 & 0.756 & 0.348 & 0.756 & 0.348 & 0.551 \\
\hline $\mathrm{MnO}$ & 2.234 & 1.802 & 1.802 & 2.234 & 2.234 & 2.234 & 1.802 & 1.802 & 2.234 & 1.802 & 2.234 & 1.802 & 2.016 \\
\hline $\mathrm{Na}_{2} \mathrm{O}$ & 13.314 & 14.012 & 12.053 & 15.145 & 12.006 & 13.163 & 15.223 & 12.008 & 12.801 & 12.006 & 12.568 & 15.223 & 13.524 \\
\hline $\mathrm{NiO}$ & 0.808 & 1.249 & 1.249 & 1.249 & 0.808 & 1.249 & 0.808 & 1.249 & 0.808 & 0.808 & 1.249 & 0.808 & 1.027 \\
\hline $\mathrm{P}_{2} \mathrm{O}_{5}$ & 0.029 & 0.029 & 0.029 & 0.131 & 0.029 & 0.029 & 0.131 & 0.131 & 0.131 & 0.131 & 0.131 & 0.029 & 0.080 \\
\hline $\mathrm{PbO}$ & 0.002 & 0.002 & 0.002 & 0.010 & 0.002 & 0.002 & 0.010 & 0.010 & 0.010 & 0.010 & 0.010 & 0.002 & 0.006 \\
\hline $\mathrm{SO}_{4}$ & 0.291 & 0.291 & 0.129 & 0.129 & 0.129 & 0.291 & 0.291 & 0.129 & 0.129 & 0.291 & 0.291 & 0.129 & 0.211 \\
\hline $\mathrm{SiO}_{2}$ & 51.154 & 50.721 & 51.154 & 50.721 & 50.721 & 51.154 & 51.154 & 51.154 & 51.154 & 50.721 & 50.721 & 50.721 & 50.936 \\
\hline $\mathrm{TiO}_{2}$ & 0.616 & 0.616 & 0.616 & 0.616 & 0.000 & 0.000 & 0.000 & 0.000 & 0.616 & 0.616 & 0.000 & 0.000 & 0.306 \\
\hline $\mathbf{U}_{3} \mathbf{O}_{8}$ & 2.483 & 2.483 & 3.090 & 2.483 & 2.483 & 3.090 & 2.483 & 2.483 & 3.090 & 3.090 & 3.090 & 3.090 & 2.785 \\
\hline $\mathrm{ZnO}$ & 0.001 & 0.001 & 0.001 & 0.004 & 0.001 & 0.001 & 0.004 & 0.004 & 0.004 & 0.004 & 0.004 & 0.001 & 0.002 \\
\hline $\mathrm{ZrO}_{2}$ & 0.011 & 0.011 & 0.011 & 0.048 & 0.011 & 0.011 & 0.048 & 0.048 & 0.048 & 0.048 & 0.048 & 0.011 & 0.029 \\
\hline
\end{tabular}


Table 2 cont. Target Glass Compositions (wt\%)

\begin{tabular}{|c|c|c|c|c|c|c|c|c|}
\hline Glass ID & 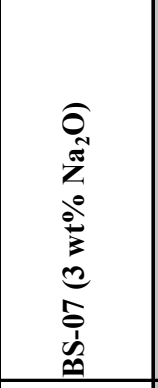 & 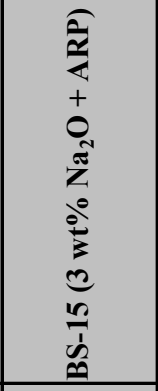 & 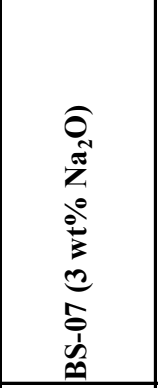 & 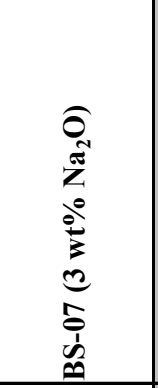 & 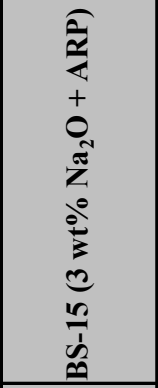 & 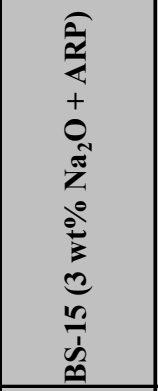 & 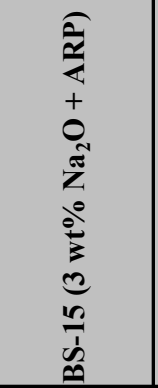 & 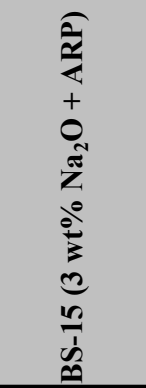 \\
\hline & SB5VS-14 & SB5VS-15 & SB5VS-16 & SB5VS-17 & SB5VS-18 & SB5VS-19 & SB5VS-20 & SB5VS-21 \\
\hline & $34 \%$ WL & $34 \%$ WL & $31 \%$ WL & 37\% WL & $25 \% \mathrm{WL}$ & $28 \%$ WL & $31 \%$ WL & $37 \% \mathrm{WL}$ \\
\hline $\mathrm{Al}_{2} \mathbf{O}_{3}$ & 7.451 & 7.183 & 6.794 & 8.109 & 5.282 & 5.916 & 6.549 & 7.817 \\
\hline $\mathbf{B}_{2} \mathbf{O}_{3}$ & 5.280 & 5.280 & 5.520 & 5.040 & 6.000 & 5.760 & 5.520 & 5.040 \\
\hline $\mathrm{BaO}$ & 0.048 & 0.047 & 0.044 & 0.053 & 0.035 & 0.039 & 0.043 & 0.051 \\
\hline $\mathrm{CaO}$ & 0.838 & 0.813 & 0.764 & 0.912 & 0.598 & 0.669 & 0.741 & 0.885 \\
\hline $\mathrm{CdO}$ & 0.033 & 0.032 & 0.030 & 0.036 & 0.023 & 0.026 & 0.029 & 0.034 \\
\hline $\mathrm{Ce}_{2} \mathrm{O}_{3}$ & 0.005 & 0.008 & 0.005 & 0.006 & 0.006 & 0.007 & 0.007 & 0.009 \\
\hline $\mathrm{Cr}_{2} \mathrm{O}_{3}$ & 0.035 & 0.035 & 0.032 & 0.038 & 0.026 & 0.029 & 0.032 & 0.038 \\
\hline $\mathrm{CuO}$ & 0.009 & 0.009 & 0.008 & 0.009 & 0.007 & 0.007 & 0.008 & 0.010 \\
\hline $\mathrm{Fe}_{2} \mathrm{O}_{3}$ & 10.075 & 9.776 & 9.186 & 10.964 & 7.188 & 8.051 & 8.913 & 10.638 \\
\hline $\mathbf{K}_{2} \mathbf{O}$ & 0.044 & 0.044 & 0.040 & 0.048 & 0.032 & 0.036 & 0.040 & 0.048 \\
\hline $\mathrm{La}_{2} \mathrm{O}_{3}$ & 0.004 & 0.005 & 0.004 & 0.004 & 0.004 & 0.004 & 0.005 & 0.006 \\
\hline $\mathbf{L i}_{\mathbf{2}} \mathbf{O}$ & 5.280 & 5.280 & 5.520 & 5.040 & 6.000 & 5.760 & 5.520 & 5.040 \\
\hline MgO & 0.551 & 0.525 & 0.502 & 0.599 & 0.386 & 0.432 & 0.479 & 0.571 \\
\hline MnO & 2.046 & 2.021 & 1.866 & 2.227 & 1.486 & 1.664 & 1.842 & 2.199 \\
\hline $\mathrm{Na}_{2} \mathrm{O}$ & 13.016 & 13.303 & 12.573 & 13.459 & 11.899 & 12.367 & 12.835 & 13.771 \\
\hline $\mathrm{NiO}$ & 1.074 & 1.043 & 0.979 & 1.169 & 0.767 & 0.859 & 0.951 & 1.135 \\
\hline $\mathbf{P}_{2} \mathbf{O}_{5}$ & 0.151 & 0.145 & 0.138 & 0.164 & 0.106 & 0.119 & 0.132 & 0.157 \\
\hline PbO & 0.004 & 0.006 & 0.004 & 0.005 & 0.005 & 0.005 & 0.006 & 0.007 \\
\hline $\mathrm{SO}_{4}$ & 0.211 & 0.249 & 0.192 & 0.230 & 0.183 & 0.205 & 0.227 & 0.271 \\
\hline $\mathrm{SiO}_{2}$ & 50.917 & 50.891 & 53.130 & 48.704 & 57.537 & 55.322 & 53.106 & 48.675 \\
\hline $\mathrm{TiO}_{2}$ & 0.003 & 0.444 & 0.003 & 0.004 & 0.327 & 0.366 & 0.405 & 0.484 \\
\hline $\mathrm{U}_{3} \mathrm{O}_{8}$ & 2.832 & 2.744 & 2.582 & 3.082 & 2.018 & 2.260 & 2.502 & 2.987 \\
\hline $\mathrm{ZnO}$ & 0.003 & 0.005 & 0.003 & 0.004 & 0.004 & 0.004 & 0.004 & 0.005 \\
\hline $\mathrm{ZrO}_{2}$ & 0.050 & 0.051 & 0.046 & 0.055 & 0.038 & 0.042 & 0.047 & 0.056 \\
\hline
\end{tabular}

\footnotetext{
${ }^{\mathrm{i}}$ The BS- 15 compositions are shaded.
} 


\subsubsection{XRD}

Representative samples of quenched and ccc glasses were submitted to AD for XRD analysis. Samples were analyzed under conditions providing a detection limit of approximately $0.5 \mathrm{vol} \%$, i.e. no crystals can be detected if the amount in the sample is less than $\sim 0.5 \mathrm{vol} \%$.

\subsubsection{PCT}

The PCT was performed in triplicate on each quenched and ccc glass to assess chemical durability using Method A of the procedure. ${ }^{10}$ Also included in the experimental test matrix was the EA glass, the Approved Reference Material (ARM) glass, and blanks from the sample cleaning batch. Samples were ground, washed, and prepared according to the standard procedure. The resulting solutions were sampled (filtered and acidified) and analyzed by AD under the auspices of two analytical plans. ${ }^{11,12}$ Samples of a multi-element, standard solution were also included in the analytical plan (as a check on the accuracy of the ICP-AES instrument). Normalized release rates were calculated based on the compositions of different compositional views using the average of the leachate concentrations.

\subsection{Results and Discussion}

\subsection{Chemical Composition Measurements}

In this section, a high-level review of the statistical analysis of the chemical composition data is provided. For a detailed discussion of the statistical analyses of the chemical composition data, the reader is referred to SRNL-L3100-2008-00012. ${ }^{13}$ The average composition for each compositional view (target, measured and measured bias corrected [bc]) are shown in Table 5 at the end of this report. Also included in the table are the relative differences between the measured or bias-corrected values and the target values. These differences are shaded when they are greater than or equal to 5\%. The results of the Batch 1 and uranium standard glass are included for reference.

The $\mathrm{Fe}_{2} \mathrm{O}_{3}$ values for the variability study glasses were consistently lower than the target values, while the values of the standard glasses were close to the reference concentrations. The range of the difference between the target and measured values was 1.6 to $11.8 \%$. These results suggest a possible batching and/or fabrication issue for the $\mathrm{Fe}_{2} \mathrm{O}_{3}$ content of the glasses. There was also scatter in the measured $\mathrm{SiO}_{2}$ values (difference of 0-6.4\%) in both the study glasses and standards, which may be due to analytical issues (i.e., dissolution technique and/or ICP-AES performance). The sum of oxides for each glass, except SB5VS-04, was within the interval of 95 to $105 \mathrm{wt} \%$. It should be noted that the oxide sum for SB5VS-04 was just below 95\%. A review of the data reveals that the measured $\mathrm{SiO}_{2}$ value for this glass is well below the target concentration, resulting in a much lower sum of oxides than expected. Although there were minor composition irregularities, the compositional analyses did not indicate any significant issues with the study glasses that would affect the conclusions drawn from this study.

\subsection{XRD}

Each of the quenched glasses was amorphous (within the detection limit of the instrument [0.5 vol\%]). A majority of the ccc samples contained visual evidence of surface crystals, but crystallization was not observed in any of the XRD patterns. Since the surface is only a small fraction of the sample and sampling for XRD is random, it is probable that the crystalline content was much below the detection 
limit of the instrument. A representative XRD pattern ${ }^{j}$ of an amorphous quenched and ccc sample is shown in Figure 1.
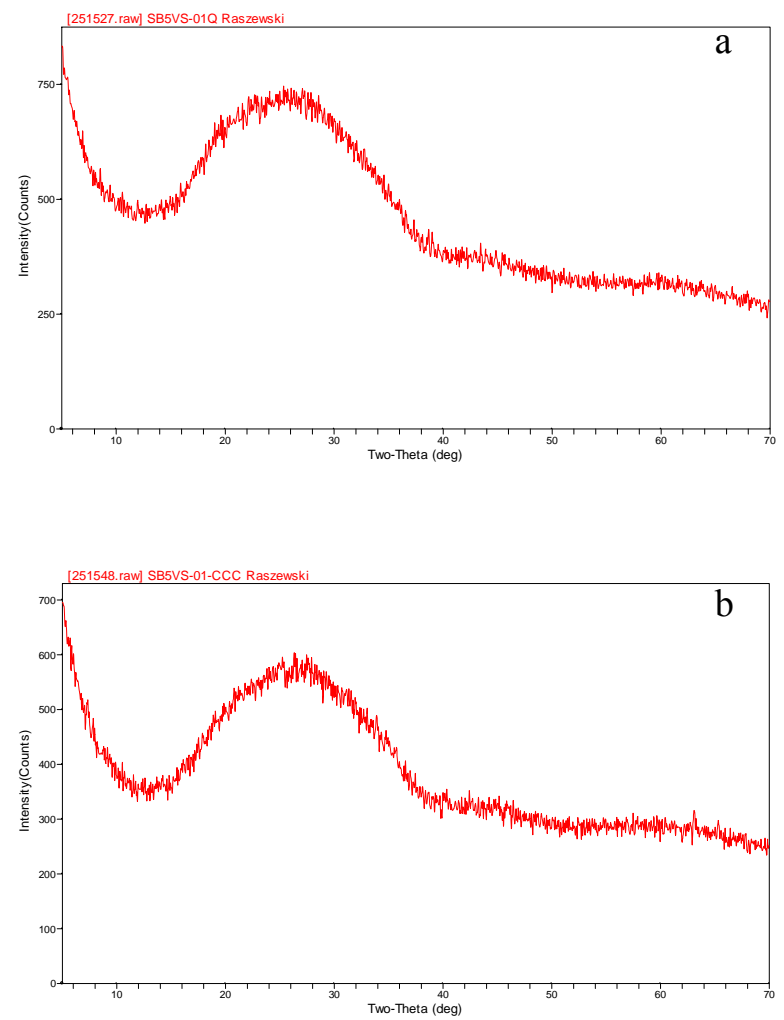

Figure 1. A representative XRD pattern for an amorphous quenched (a) and ccc (b) sample.

\subsection{PCT}

As with the chemical composition information, this section will only provide a high-level review of the durability data. For a complete discussion of the statistical analyses of the PCT data see SRNL-L31002008-00012. ${ }^{13}$ Normalized PCT data are shown in Table 6 at the end of this report. AE and ARM results are included for reference.

The normalized boron release $(\mathrm{NL}[\mathrm{B}(\mathrm{g} / \mathrm{L})]$ was in the range of $0.63-0.97 \mathrm{~g} / \mathrm{L}$ for the quenched glasses and $0.62-0.98 \mathrm{~g} / \mathrm{L}$ for the ccc glasses (regardless of compositional view). There was no indication of a dramatic heat treatment impact for any of the SB5 VS glasses. The PCTs for all of the glasses, even those glasses that were chosen to challenge the homogeneity constraint (SB5VS-18 and SB5VS-19), were acceptable based upon comparisons to the EA glass $(16.695 \mathrm{~g} / \mathrm{L})$ for all compositional views. The glasses are also predictable with respect to the durability models as shown in Figure 2. The points plotted in the figure cover all compositional views as well as both heat treatments, which are within the $95 \%$ confidence interval. EA and ARM results are also indicated on the plot for reference. The small cluster of points closer to the edge of the confidence band was generated by bounding sludge compositions that led to

\footnotetext{
${ }^{\mathrm{j}}$ The remainder of the XRD patterns (quenched and ccc) can be viewed in the laboratory notebook for the SB5 variability study on pages 30-33 (WSRC-NB-2007-00002).
} 
glasses with more negative $\mathrm{B}$ del $\mathrm{G}_{\mathrm{p}}$ values than the other glasses. ${ }^{\mathrm{k}}$

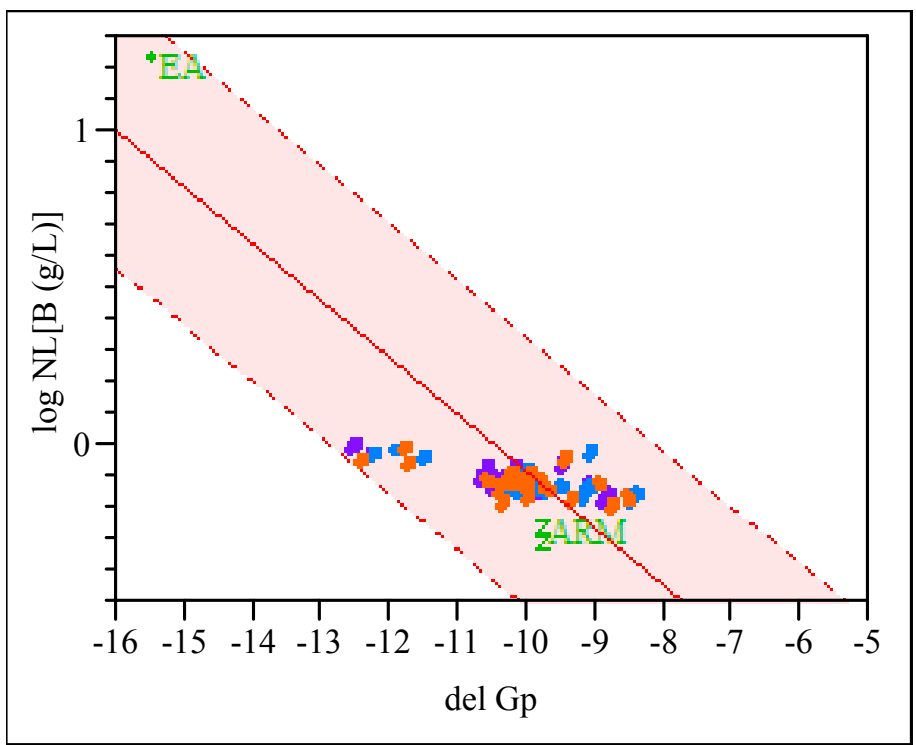

Figure 2. $\log N L[B(g / L)]$ versus del $G_{p}\left(\Delta G_{p}\right)$ model with a $95 \%$ confidence interval for individual PCTs.

\subsection{Conclusions}

Frit 418 is a viable option for the processing of SB5 for both sludge-only and coupled operations. The addition of ARP did not have any negative impacts on the acceptability and predictability of the variability study glasses.

Based on the measured PCT response, all of the glasses are acceptable as compared to the Environmental Assessment (EA) reference glass regardless of the thermal history and predictable using the current PCCS model for durability.

The homogeneity constraint can be ignored for SB5 for coupled operations (based on the Appendix J compositional information), as both of the glasses (SB5VS-18 and SB5VS-19) had durabilities that were acceptable with respect to the reference EA glass.

\subsection{Acknowledgements}

The authors would like to thank AD, David Best, Holly Hall, Irene Reamer and Pat Simmons for support on this task.

\footnotetext{
${ }^{\mathrm{k}}$ Specifically these glasses are SB5VS-04, SB5VS-07 and SB5VS-12, which were predicted to have B del $G_{P}$ values of $-12.506,-12.551$ and -12.280 , respectively. See Display 2 of SRNL-L3100-2008-00012 for more details.
} 
Table 5. Summary of the Target, Average Measured/Bias-Corrected Chemical Compositions

\begin{tabular}{|c|c|c|c|c|c|c|c|c|c|}
\hline & & & & Measured & & & & & \\
\hline & Glass & & Measured & Bias-Corrected & Targeted & Diff of & Diff of & $\%$ Diff of & $\%$ Diff of \\
\hline Glass \# & ID & Oxide & $(w t \%)$ & $(w t \%)$ & $(w t \%)$ & Measured & Meas BC & Measured & Meas BC \\
\hline 1 & SB5VS-01 & $\mathrm{Al} 2 \mathrm{O} 3(\mathrm{wt} \%)$ & 6.4337 & 6.5207 & 6.4522 & -0.0185 & 0.0684 & $-0.3 \%$ & $1.1 \%$ \\
\hline 1 & SB5VS-01 & $\mathrm{B} 2 \mathrm{O} 3(\mathrm{wt} \%)$ & 5.4175 & 5.6550 & 5.2800 & 0.1375 & 0.3750 & $2.6 \%$ & $7.1 \%$ \\
\hline 1 & SB5VS-01 & $\mathrm{BaO}(\mathrm{wt} \%)$ & 0.0106 & 0.0103 & 0.0099 & 0.0007 & 0.0004 & $7.0 \%$ & $3.6 \%$ \\
\hline 1 & SB5VS-01 & $\mathrm{CaO}(\mathrm{wt} \%)$ & 0.6076 & 0.6111 & 0.6256 & -0.0180 & -0.0146 & $-2.9 \%$ & $-2.3 \%$ \\
\hline 1 & SB5VS-01 & $\mathrm{CdO}(\mathrm{wt} \%)$ & 0.0057 & 0.0057 & 0.0064 & -0.0007 & -0.0007 & $-10.8 \%$ & $-10.8 \%$ \\
\hline 1 & SB5VS-01 & $\mathrm{Ce} 2 \mathrm{O} 3(\mathrm{wt} \%)$ & 0.0205 & 0.0205 & 0.0027 & 0.0178 & 0.0178 & $648.4 \%$ & $648.4 \%$ \\
\hline 1 & SB5VS-01 & $\mathrm{Cr} 2 \mathrm{O} 3(\mathrm{wt} \%)$ & 0.0091 & 0.0090 & 0.0094 & -0.0003 & -0.0004 & $-2.9 \%$ & $-4.6 \%$ \\
\hline 1 & SB5VS-01 & $\mathrm{CuO}(\mathrm{wt} \%)$ & 0.0113 & 0.0112 & 0.0027 & 0.0085 & 0.0085 & $312.1 \%$ & $310.7 \%$ \\
\hline 1 & SB5VS-01 & $\mathrm{Fe} 2 \mathrm{O} 3(\mathrm{wt} \%)$ & 10.6012 & 10.6336 & 11.0159 & -0.4147 & -0.3823 & $-3.8 \%$ & $-3.5 \%$ \\
\hline 1 & SB5VS-01 & $\mathrm{K} 2 \mathrm{O}(\mathrm{wt} \%)$ & 0.0205 & 0.0202 & 0.0100 & 0.0105 & 0.0102 & $105.1 \%$ & $102.1 \%$ \\
\hline 1 & SB5VS-01 & $\mathrm{La} 2 \mathrm{O} 3(\mathrm{wt} \%)$ & 0.0001 & 0.0001 & 0.0020 & -0.0019 & -0.0019 & $-94.1 \%$ & $-94.1 \%$ \\
\hline 1 & SB5VS-01 & $\mathrm{Li} 2 \mathrm{O}(\mathrm{wt} \%)$ & 4.7310 & 5.3037 & 5.2810 & -0.5500 & 0.0227 & $-10.4 \%$ & $0.4 \%$ \\
\hline 1 & SB5VS-01 & $\mathrm{MgO}(\mathrm{wt} \%)$ & 0.3540 & 0.3412 & 0.3483 & 0.0057 & -0.0071 & $1.6 \%$ & $-2.0 \%$ \\
\hline 1 & SB5VS-01 & $\mathrm{MnO}(\mathrm{wt} \%)$ & 2.2144 & 2.2119 & 2.2344 & -0.0200 & -0.0225 & $-0.9 \%$ & $-1.0 \%$ \\
\hline 1 & SB5VS-01 & $\mathrm{Na} 2 \mathrm{O}(\mathrm{wt} \%)$ & 13.3856 & 13.2278 & 13.3141 & 0.0716 & -0.0863 & $0.5 \%$ & $-0.6 \%$ \\
\hline 1 & $\begin{array}{l}\text { SB5VS-01 } \\
\text { SBVS }\end{array}$ & $\mathrm{NiO}(\mathrm{wt} \%)$ & 0.7670 & 0.7536 & 0.8081 & -0.0411 & -0.0545 & $-5.1 \%$ & $-6.7 \%$ \\
\hline 1 & SB5VS-01 & $\mathrm{P} 2 \mathrm{O} 5(\mathrm{wt} \%)$ & 0.0206 & 0.0206 & 0.0290 & $\begin{array}{l}-0.0083 \\
\end{array}$ & -0.0083 & $-28.8 \%$ & $-28.8 \%$ \\
\hline 1 & SB5VS-01 & $\mathrm{PbO}(\mathrm{wt} \%)$ & 0.0073 & 0.0073 & 0.0022 & 0.0050 & 0.0050 & $226.5 \%$ & $226.5 \%$ \\
\hline 1 & SB5VS-01 & $\mathrm{SiO} 2(\mathrm{wt} \%)$ & 50.8084 & 50.5041 & 51.1540 & -0.3456 & -0.6499 & $-0.7 \%$ & $-1.3 \%$ \\
\hline 1 & SB5VS-01 & SO4 (wt\%) & 0.3161 & 0.3161 & 0.2913 & 0.0247 & 0.0247 & $8.5 \%$ & $8.5 \%$ \\
\hline 1 & SB5VS-01 & $\mathrm{TiO} 2(\mathrm{wt} \%)$ & 0.5996 & 0.5884 & 0.6164 & -0.0168 & -0.0280 & $-2.7 \%$ & $-4.5 \%$ \\
\hline 1 & SB5VS-01 & U3O8 (wt\%) & 2.3613 & 2.4210 & 2.4828 & -0.1215 & -0.0618 & $-4.9 \%$ & $-2.5 \%$ \\
\hline 1 & SB5VS-01 & $\mathrm{ZnO}(\mathrm{wt} \%)$ & 0.0084 & 0.0084 & 0.0008 & 0.0076 & 0.0076 & $902.7 \%$ & $902.7 \%$ \\
\hline 1 & SB5VS-01 & $\mathrm{ZrO} 2(\mathrm{wt} \%)$ & 0.0172 & 0.0172 & 0.0107 & 0.0066 & 0.0066 & $61.5 \%$ & $61.5 \%$ \\
\hline 1 & SB5VS-01 & Sum & 98.7289 & 99.2186 & 99.9901 & -1.2612 & -0.7715 & $-1.3 \%$ & $-0.8 \%$ \\
\hline 2 & SB5VS-02 & $\mathrm{Al} 2 \mathrm{O} 3(\mathrm{wt} \%)$ & 7.8036 & 7.8834 & 8.1889 & -0.3852 & -0.3055 & $-4.7 \%$ & $-3.7 \%$ \\
\hline 2 & SB5VS-02 & $\mathrm{B} 2 \mathrm{O} 3(\mathrm{wt} \%)$ & 5.4658 & 5.3960 & 5.2800 & 0.1858 & 0.1160 & $3.5 \%$ & $2.2 \%$ \\
\hline 2 & SB5VS-02 & $\mathrm{BaO}(\mathrm{wt} \%)$ & 0.0106 & 0.0101 & 0.0099 & 0.0007 & 0.0002 & $7.0 \%$ & $2.0 \%$ \\
\hline 2 & SB5VS-02 & $\mathrm{CaO}(\mathrm{wt} \%)$ & 0.5975 & 0.5960 & 0.6256 & -0.0282 & -0.0296 & $-4.5 \%$ & $-4.7 \%$ \\
\hline 2 & SB5VS-02 & $\mathrm{CdO}(\mathrm{wt} \%)$ & 0.0051 & 0.0051 & 0.0064 & -0.0013 & $\begin{array}{l}-0.0013 \\
\end{array}$ & $-19.7 \%$ & $-19.7 \%$ \\
\hline 2 & SB5VS-02 & $\mathrm{Ce} 2 \mathrm{O} 3(\mathrm{wt} \%)$ & 0.0234 & 0.0234 & 0.0027 & 0.0207 & 0.0207 & $755.3 \%$ & $755.3 \%$ \\
\hline 2 & SB5VS-02 & $\mathrm{Cr} 2 \mathrm{O} 3(\mathrm{wt} \%)$ & 0.0102 & 0.0097 & 0.0094 & 0.0008 & 0.0003 & $8.8 \%$ & $2.8 \%$ \\
\hline 2 & SB5VS-02 & $\mathrm{CuO}(\mathrm{wt} \%)$ & 0.0119 & 0.0115 & 0.0027 & 0.0092 & 0.0088 & $335.0 \%$ & $321.6 \%$ \\
\hline 2 & SB5VS-02 & $\mathrm{Fe} 2 \mathrm{O} 3(\mathrm{wt} \%)$ & 8.0135 & 7.9532 & 8.5992 & -0.5857 & -0.6461 & $-6.8 \%$ & $-7.5 \%$ \\
\hline 2 & SB5VS-02 & $\mathrm{K} 2 \mathrm{O}(\mathrm{wt} \%)$ & 0.0145 & 0.0144 & 0.0100 & 0.0045 & 0.0044 & $44.8 \%$ & $44.1 \%$ \\
\hline 2 & SB5VS-02 & $\mathrm{La} 2 \mathrm{O} 3(\mathrm{wt} \%)$ & 0.0001 & 0.0001 & 0.0020 & -0.0019 & -0.0019 & $-94.1 \%$ & $-94.1 \%$ \\
\hline 2 & SB5VS-02 & $\mathrm{Li} 2 \mathrm{O}(\mathrm{wt} \%)$ & 5.0001 & 5.4506 & 5.2810 & -0.2809 & 0.1696 & $-5.3 \%$ & $3.2 \%$ \\
\hline 2 & SB5VS-02 & $\mathrm{MgO}(\mathrm{wt} \%)$ & 0.7392 & 0.7215 & 0.7559 & -0.0167 & -0.0344 & $-2.2 \%$ & $-4.6 \%$ \\
\hline 2 & SB5VS-02 & $\mathrm{MnO}(\mathrm{wt} \%)$ & 1.7560 & 1.7133 & 1.8016 & -0.0456 & -0.0883 & $-2.5 \%$ & $-4.9 \%$ \\
\hline 2 & SB5VS-02 & $\mathrm{Na} 2 \mathrm{O}(\mathrm{wt} \%)$ & 13.3418 & 13.0051 & 14.0118 & -0.6699 & -1.0067 & $-4.8 \%$ & $-7.2 \%$ \\
\hline 2 & SB5VS-02 & $\mathrm{NiO}(\mathrm{wt} \%)$ & 1.1618 & 1.1116 & 1.2490 & -0.0872 & -0.1374 & $-7.0 \%$ & $-11.0 \%$ \\
\hline 2 & SB5VS-02 & $\mathrm{P} 2 \mathrm{O} 5(\mathrm{wt} \%)$ & 0.0203 & 0.0203 & 0.0290 & -0.0086 & $\begin{array}{l}-0.0086 \\
\end{array}$ & $-29.9 \%$ & $-29.9 \%$ \\
\hline 2 & SB5VS-02 & $\mathrm{PbO}(\mathrm{wt} \%)$ & 0.0073 & 0.0073 & 0.0022 & 0.0050 & 0.0050 & $226.5 \%$ & $226.5 \%$ \\
\hline 2 & SB5VS-02 & $\mathrm{SiO} 2(\mathrm{wt} \%)$ & 47.9738 & 50.1351 & 50.7210 & -2.7472 & -0.5859 & $-5.4 \%$ & $-1.2 \%$ \\
\hline 2 & SB5VS-02 & SO4 (wt\%) & 0.2876 & 0.2876 & 0.2913 & -0.0037 & -0.0037 & $-1.3 \%$ & $-1.3 \%$ \\
\hline 2 & SB5VS-02 & TiO2 (wt $\%)$ & 0.5971 & 0.5850 & 0.6164 & -0.0193 & -0.0314 & $-3.1 \%$ & $-5.1 \%$ \\
\hline 2 & SB5VS-02 & U3O8 (wt\%) & 2.2670 & 2.3283 & 2.4828 & -0.2158 & -0.1545 & $-8.7 \%$ & $-6.2 \%$ \\
\hline 2 & SB5VS-02 & $\mathrm{ZnO}(\mathrm{wt} \%)$ & 0.0115 & 0.0115 & 0.0008 & 0.0107 & 0.0107 & $1274.0 \%$ & $1274.0 \%$ \\
\hline 2 & SB5VS-02 & $\mathrm{ZrO} 2(\mathrm{wt} \%)$ & 0.0149 & 0.0149 & 0.0107 & 0.0042 & 0.0042 & $39.3 \%$ & $39.3 \%$ \\
\hline 2 & SB5VS-02 & Sum & 95.1347 & 97.2949 & 99.9904 & -4.8557 & -2.6955 & $-4.9 \%$ & $-2.7 \%$ \\
\hline
\end{tabular}


Table 5 cont. Summary of the Target, Average Measured/Bias-Corrected Chemical Compositions

\begin{tabular}{|c|c|c|c|c|c|c|c|c|c|}
\hline & & & & Measured & & & & & \\
\hline & Glass & & Measured & Bias-Corrected & Targeted & Diff of & Diff of & $\%$ Diff of & $\%$ Diff of \\
\hline Glass \# & ID & Oxide & $(w t \%)$ & $(w t \%)$ & $(w t \%)$ & Measured & Meas BC & Measured & Meas BC \\
\hline 3 & SB5VS-03 & $\mathrm{Al} 2 \mathrm{O} 3(\mathrm{wt} \%)$ & 6.3582 & 6.4442 & 6.4522 & -0.0941 & -0.0081 & $-1.5 \%$ & $-0.1 \%$ \\
\hline 3 & SB5VS-03 & $\mathrm{B} 2 \mathrm{O} 3(\mathrm{wt} \%)$ & 4.8138 & 5.0260 & 5.2800 & -0.4662 & -0.2540 & $-8.8 \%$ & $-4.8 \%$ \\
\hline 3 & SB5VS-03 & $\mathrm{BaO}(\mathrm{wt} \%)$ & 0.0100 & 0.0097 & 0.0099 & 0.0001 & -0.0002 & $1.3 \%$ & $-1.9 \%$ \\
\hline 3 & SB5VS-03 & $\mathrm{CaO}(\mathrm{wt} \%)$ & 1.0004 & 1.0061 & 1.0255 & -0.0251 & -0.0194 & $-2.4 \%$ & $-1.9 \%$ \\
\hline 3 & SB5VS-03 & $\mathrm{CdO}(\mathrm{wt} \%)$ & 0.0046 & 0.0046 & 0.0064 & $\begin{array}{l}-0.0018 \\
\end{array}$ & $\begin{array}{l}-0.0018 \\
\end{array}$ & $-28.7 \%$ & $-28.7 \%$ \\
\hline 3 & SB5VS-03 & $\mathrm{Ce} 2 \mathrm{O} 3(\mathrm{wt} \%)$ & 0.0243 & 0.0243 & 0.0027 & 0.0216 & 0.0216 & $787.3 \%$ & $787.3 \%$ \\
\hline 3 & SB5VS-03 & $\mathrm{Cr} 2 \mathrm{O} 3(\mathrm{wt} \%)$ & 0.0117 & 0.0115 & 0.0094 & 0.0023 & 0.0021 & $24.3 \%$ & $22.2 \%$ \\
\hline 3 & SB5VS-03 & $\mathrm{CuO}(\mathrm{wt} \%)$ & 0.0128 & 0.0128 & 0.0027 & 0.0101 & 0.0101 & $369.3 \%$ & $367.7 \%$ \\
\hline 3 & SB5VS-03 & $\mathrm{Fe} 2 \mathrm{O} 3(\mathrm{wt} \%)$ & 10.5655 & 10.5971 & 11.0159 & -0.4504 & -0.4188 & $-4.1 \%$ & $-3.8 \%$ \\
\hline 3 & SB5VS-03 & $\mathrm{K} 2 \mathrm{O}(\mathrm{wt} \%)$ & 0.0169 & 0.0166 & 0.0100 & 0.0069 & 0.0066 & $68.9 \%$ & $66.4 \%$ \\
\hline 3 & SB5VS-03 & $\mathrm{La} 2 \mathrm{O} 3(\mathrm{wt} \%)$ & 0.0001 & 0.0001 & 0.0020 & -0.0019 & -0.0019 & $-94.1 \%$ & $-94.1 \%$ \\
\hline 3 & SB5VS-03 & $\mathrm{Li} 2 \mathrm{O}(\mathrm{wt} \%)$ & 4.6610 & 5.2260 & 5.2810 & -0.6200 & -0.0550 & $-11.7 \%$ & $-1.0 \%$ \\
\hline 3 & SB5VS-03 & $\mathrm{MgO}(\mathrm{wt} \%)$ & 0.7802 & 0.7519 & 0.7559 & 0.0243 & -0.0040 & $3.2 \%$ & $-0.5 \%$ \\
\hline 3 & SB5VS-03 & $\mathrm{MnO}(\mathrm{wt} \%)$ & 1.7657 & 1.7636 & 1.8016 & -0.0359 & -0.0380 & $-2.0 \%$ & $-2.1 \%$ \\
\hline 3 & SB5VS-03 & $\mathrm{Na} 2 \mathrm{O}$ (wt\%) & 11.9332 & 11.7927 & 12.0533 & -0.1202 & -0.2606 & $-1.0 \%$ & $-2.2 \%$ \\
\hline 3 & SB5VS-03 & $\mathrm{NiO}(\mathrm{wt} \%)$ & 1.1834 & 1.1628 & 1.2490 & -0.0656 & -0.0862 & $-5.3 \%$ & $-6.9 \%$ \\
\hline 3 & SB5VS-03 & P2O5 (wt\%) & 0.0206 & 0.0206 & 0.0290 & -0.0083 & -0.0083 & $-28.8 \%$ & $-28.8 \%$ \\
\hline 3 & SB5VS-03 & $\mathrm{PbO}(\mathrm{wt} \%)$ & 0.0083 & 0.0083 & 0.0022 & 0.0061 & 0.0061 & $274.9 \%$ & $274.9 \%$ \\
\hline 3 & SB5VS-03 & $\mathrm{SiO} 2(\mathrm{wt} \%)$ & 48.1877 & 47.9051 & 51.1540 & -2.9663 & -3.2489 & $-5.8 \%$ & $-6.4 \%$ \\
\hline 3 & SB5VS-03 & $\mathrm{SO} 4(\mathrm{wt} \%)$ & 0.1753 & 0.1753 & 0.1293 & 0.0460 & 0.0460 & $35.6 \%$ & $35.6 \%$ \\
\hline 3 & SB5VS-03 & $\mathrm{TiO} 2(\mathrm{wt} \%)$ & 0.6205 & 0.6088 & 0.6164 & 0.0041 & -0.0076 & $0.7 \%$ & $-1.2 \%$ \\
\hline 3 & SB5VS-03 & U3O8 (wt\%) & 2.9008 & 2.9743 & 3.0900 & -0.1892 & -0.1157 & $-6.1 \%$ & $-3.7 \%$ \\
\hline 3 & SB5VS-03 & $\mathrm{ZnO}(\mathrm{wt} \%)$ & 0.0096 & 0.0096 & 0.0008 & 0.0088 & 0.0088 & $1051.2 \%$ & $1051.2 \%$ \\
\hline 3 & SB5VS-03 & $\mathrm{ZrO} 2(\mathrm{wt} \%)$ & 0.0155 & 0.0155 & 0.0107 & 0.0049 & 0.0049 & $45.7 \%$ & $45.7 \%$ \\
\hline 3 & SB5VS-03 & Sum & 95.0803 & 95.5677 & 99.9901 & -4.9098 & -4.4224 & $-4.9 \%$ & $-4.4 \%$ \\
\hline 4 & SB5VS-04 & $\mathrm{Al} 2 \mathrm{O} 3(\mathrm{wt} \%)$ & 6.2165 & 6.2800 & 6.4522 & -0.2358 & -0.1723 & $-3.7 \%$ & $-2.7 \%$ \\
\hline 4 & SB5VS-04 & $\mathrm{B} 2 \mathrm{O} 3(\mathrm{wt} \%)$ & 5.4577 & 5.3885 & 5.2800 & 0.1777 & 0.1085 & $3.4 \%$ & $2.1 \%$ \\
\hline 4 & SB5VS-04 & $\mathrm{BaO}(\mathrm{wt} \%)$ & 0.0449 & 0.0429 & 0.0448 & 0.0002 & $\begin{array}{l}-0.0019 \\
\end{array}$ & $0.4 \%$ & $-4.3 \%$ \\
\hline 4 & SB5VS-04 & $\mathrm{CaO}(\mathrm{wt} \%)$ & 0.9899 & 0.9876 & 1.0255 & -0.0356 & -0.0380 & $-3.5 \%$ & $-3.7 \%$ \\
\hline 4 & SB5VS-04 & $\mathrm{CdO}(\mathrm{wt} \%)$ & 0.0257 & 0.0257 & 0.0289 & -0.0032 & -0.0032 & $-11.1 \%$ & $-11.1 \%$ \\
\hline 4 & SB5VS-04 & $\mathrm{Ce} 2 \mathrm{O} 3(\mathrm{wt} \%)$ & 0.0293 & 0.0293 & 0.0124 & 0.0169 & 0.0169 & $136.7 \%$ & $136.7 \%$ \\
\hline 4 & SB5VS-04 & $\mathrm{Cr} 2 \mathrm{O} 3(\mathrm{wt} \%)$ & 0.0420 & 0.0397 & 0.0425 & -0.0005 & -0.0028 & $-1.1 \%$ & $-6.6 \%$ \\
\hline 4 & SB5VS-04 & $\mathrm{CuO}(\mathrm{wt} \%)$ & 0.0194 & 0.0188 & 0.0123 & 0.0071 & 0.0065 & $57.2 \%$ & $52.3 \%$ \\
\hline 4 & SB5VS-04 & $\mathrm{Fe} 2 \mathrm{O} 3(\mathrm{wt} \%)$ & 8.1171 & 8.0560 & 8.5992 & -0.4821 & -0.5432 & $-5.6 \%$ & $-6.3 \%$ \\
\hline 4 & SB5VS-04 & $\mathrm{K} 2 \mathrm{O}(\mathrm{wt} \%)$ & 0.0458 & 0.0456 & 0.0451 & 0.0007 & 0.0005 & $1.5 \%$ & $1.0 \%$ \\
\hline 4 & SB5VS-04 & $\mathrm{La} 2 \mathrm{O} 3(\mathrm{wt} \%)$ & 0.0029 & 0.0029 & 0.0089 & -0.0060 & -0.0060 & $-67.2 \%$ & $-67.2 \%$ \\
\hline 4 & SB5VS-04 & $\mathrm{Li} 2 \mathrm{O}(\mathrm{wt} \%)$ & 4.8440 & 5.2825 & 5.2840 & -0.4400 & -0.0015 & $-8.3 \%$ & $0.0 \%$ \\
\hline 4 & SB5VS-04 & $\mathrm{MgO}(\mathrm{wt} \%)$ & 0.3441 & 0.3359 & 0.3483 & -0.0042 & -0.0125 & $-1.2 \%$ & $-3.6 \%$ \\
\hline 4 & SB5VS-04 & $\mathrm{MnO}\left(\mathrm{wt}^{2} \%\right)$ & 2.1692 & 2.1168 & 2.2344 & -0.0652 & -0.1176 & $-2.9 \%$ & $-5.3 \%$ \\
\hline 4 & SB5VS-04 & $\mathrm{Na} 2 \mathrm{O}(\mathrm{wt} \%)$ & 14.4910 & 14.1253 & 15.1453 & -0.6543 & -1.0201 & $-4.3 \%$ & $-6.7 \%$ \\
\hline 4 & SB5VS-04 & $\mathrm{NiO}(\mathrm{wt} \%)$ & 1.2047 & 1.1527 & 1.2490 & -0.0443 & -0.0963 & $-3.5 \%$ & $-7.7 \%$ \\
\hline 4 & SB5VS-04 & $\mathrm{P} 2 \mathrm{O} 5(\mathrm{wt} \%)$ & 0.0481 & 0.0481 & 0.1308 & -0.0826 & -0.0826 & $-63.2 \%$ & $-63.2 \%$ \\
\hline 4 & SB5VS-04 & $\mathrm{PbO}(\mathrm{wt} \%)$ & 0.0127 & 0.0127 & 0.0101 & 0.0026 & 0.0026 & $25.9 \%$ & $25.9 \%$ \\
\hline 4 & SB5VS-04 & $\mathrm{SiO} 2(\mathrm{wt} \%)$ & 47.3855 & 49.5195 & 50.7210 & -3.3355 & -1.2015 & $-6.6 \%$ & $-2.4 \%$ \\
\hline 4 & SB5VS-04 & SO4 (wt\%) & 0.1850 & 0.1850 & 0.1293 & 0.0557 & 0.0557 & $43.1 \%$ & $43.1 \%$ \\
\hline 4 & SB5VS-04 & $\mathrm{TiO} 2(\mathrm{wt} \%)$ & 0.6101 & 0.5976 & 0.6164 & -0.0063 & -0.0188 & $-1.0 \%$ & $-3.0 \%$ \\
\hline 4 & SB5VS-04 & U3O8 (wt\%) & 2.3142 & 2.3767 & 2.4828 & -0.1686 & -0.1062 & $-6.8 \%$ & $-4.3 \%$ \\
\hline 4 & SB5VS-04 & $\mathrm{ZnO}(\mathrm{wt} \%)$ & 0.0106 & 0.0106 & 0.0038 & 0.0068 & 0.0068 & $179.5 \%$ & $179.5 \%$ \\
\hline 4 & SB5VS-04 & $\mathrm{ZrO} 2(\mathrm{wt} \%)$ & 0.0486 & 0.0486 & 0.0482 & 0.0005 & 0.0005 & $1.0 \%$ & $1.0 \%$ \\
\hline 4 & SB5VS-04 & Sum & 94.6591 & 96.7289 & 99.9552 & $\begin{array}{l}-5.2961 \\
\end{array}$ & -3.2264 & $-5.3 \%$ & $-3.2 \%$ \\
\hline
\end{tabular}


Table 5 cont. Summary of the Target, Average Measured/Bias-Corrected Chemical Compositions

\begin{tabular}{|c|c|c|c|c|c|c|c|c|c|}
\hline & & & & Measured & & & & & \\
\hline & Glass & & Measured & Bias-Corrected & Targeted & Diff of & Diff of & $\%$ Diff of & $\%$ Diff of \\
\hline Glass \# & ID & Oxide & $(w t \%)$ & $(w t \%)$ & $(w t \%)$ & Measured & Meas BC & Measured & Meas BC \\
\hline 5 & SB5VS-05 & $\mathrm{Al} 2 \mathrm{O} 3(\mathrm{wt} \%)$ & 7.9359 & 8.0170 & 8.1889 & -0.2530 & -0.1718 & $-3.1 \%$ & $-2.1 \%$ \\
\hline 5 & SB5VS-05 & $\mathrm{B} 2 \mathrm{O} 3(\mathrm{wt} \%)$ & 5.3611 & 5.2923 & 5.2800 & 0.0811 & 0.0123 & $1.5 \%$ & $0.2 \%$ \\
\hline 5 & SB5VS-05 & $\mathrm{BaO}(\mathrm{wt} \%)$ & 0.0100 & 0.0096 & 0.0099 & 0.0001 & -0.0003 & $1.3 \%$ & $-3.4 \%$ \\
\hline 5 & SB5VS-05 & $\mathrm{CaO}(\mathrm{wt} \%)$ & 1.0036 & 1.0012 & 1.0255 & -0.0220 & -0.0243 & $-2.1 \%$ & $-2.4 \%$ \\
\hline 5 & SB5VS-05 & $\mathrm{CdO}(\mathrm{wt} \%)$ & 0.0051 & 0.0051 & 0.0064 & -0.0013 & -0.0013 & $-19.7 \%$ & $-19.7 \%$ \\
\hline 5 & SB5VS-05 & $\mathrm{Ce} 2 \mathrm{O} 3(\mathrm{wt} \%)$ & 0.0193 & 0.0193 & 0.0027 & 0.0166 & 0.0166 & $605.6 \%$ & $605.6 \%$ \\
\hline 5 & SB5VS-05 & $\mathrm{Cr} 2 \mathrm{O} 3(\mathrm{wt} \%)$ & 0.0124 & 0.0117 & 0.0094 & 0.0030 & 0.0023 & $32.1 \%$ & $24.8 \%$ \\
\hline 5 & SB5VS-05 & $\mathrm{CuO}(\mathrm{wt} \%)$ & 0.0119 & 0.0115 & 0.0027 & 0.0092 & 0.0088 & $335.0 \%$ & $321.6 \%$ \\
\hline 5 & SB5VS-05 & $\mathrm{Fe} 2 \mathrm{O} 3(\mathrm{wt} \%)$ & 10.3832 & 10.3050 & 10.9921 & -0.6089 & -0.6870 & $-5.5 \%$ & $-6.3 \%$ \\
\hline 5 & SB5VS-05 & $\mathrm{K} 2 \mathrm{O}(\mathrm{wt} \%)$ & 0.0166 & 0.0165 & 0.0100 & 0.0066 & 0.0065 & $65.9 \%$ & $65.1 \%$ \\
\hline 5 & SB5VS-05 & $\mathrm{La} 2 \mathrm{O} 3(\mathrm{wt} \%)$ & 0.0001 & 0.0001 & 0.0020 & -0.0019 & -0.0019 & $-94.1 \%$ & $-94.1 \%$ \\
\hline 5 & SB5VS-05 & $\mathrm{Li} 2 \mathrm{O}(\mathrm{wt} \%)$ & 4.9732 & 5.4218 & 5.2810 & -0.3078 & 0.1408 & $-5.8 \%$ & $2.7 \%$ \\
\hline 5 & SB5VS-05 & $\mathrm{MgO}(\mathrm{wt} \%)$ & 0.7529 & 0.7348 & 0.7559 & -0.0030 & -0.0211 & $-0.4 \%$ & $-2.8 \%$ \\
\hline 5 & SB5VS-05 & $\mathrm{MnO}(\mathrm{wt} \%)$ & 2.1595 & 2.1077 & 2.2344 & -0.0749 & -0.1267 & $-3.4 \%$ & $-5.7 \%$ \\
\hline 5 & SB5VS-05 & $\mathrm{Na} 2 \mathrm{O}$ (wt\%) & 11.9096 & 11.6090 & 12.0056 & -0.0960 & -0.3966 & $-0.8 \%$ & $-3.3 \%$ \\
\hline 5 & SB5VS-05 & $\mathrm{NiO}(\mathrm{wt} \%)$ & 0.7597 & 0.7268 & 0.8081 & -0.0484 & -0.0813 & $-6.0 \%$ & $-10.1 \%$ \\
\hline 5 & SB5VS-05 & P2O5 (wt\%) & 0.0206 & 0.0206 & 0.0290 & -0.0083 & -0.0083 & $-28.8 \%$ & $-28.8 \%$ \\
\hline 5 & SB5VS-05 & $\mathrm{PbO}(\mathrm{wt} \%)$ & 0.0105 & 0.0105 & 0.0022 & 0.0083 & 0.0083 & $371.6 \%$ & $371.6 \%$ \\
\hline 5 & SB5VS-05 & $\mathrm{SiO} 2(\mathrm{wt} \%)$ & 48.4017 & 50.5777 & 50.7210 & -2.3193 & $\begin{array}{l}-0.1433 \\
\end{array}$ & $-4.6 \%$ & $-0.3 \%$ \\
\hline 5 & SB5VS-05 & $\mathrm{SO} 4(\mathrm{wt} \%)$ & 0.2464 & 0.2464 & 0.1293 & 0.1172 & 0.1172 & $90.6 \%$ & $90.6 \%$ \\
\hline 5 & SB5VS-05 & $\mathrm{TiO} 2(\mathrm{wt} \%)$ & 0.0083 & 0.0082 & 0.0000 & 0.0083 & 0.0082 & & \\
\hline 5 & SB5VS-05 & U3O8 (wt\%) & 2.3407 & 2.4039 & 2.4828 & -0.1421 & -0.0789 & $-5.7 \%$ & $-3.2 \%$ \\
\hline 5 & SB5VS-05 & $\mathrm{ZnO}(\mathrm{wt} \%)$ & 0.0084 & 0.0084 & 0.0008 & 0.0076 & 0.0076 & $902.7 \%$ & $902.7 \%$ \\
\hline 5 & SB5VS-05 & $\mathrm{ZrO} 2(\mathrm{wt} \%)$ & 0.0149 & 0.0149 & 0.0107 & 0.0042 & 0.0042 & $39.3 \%$ & $39.3 \%$ \\
\hline 5 & SB5VS-05 & Sum & 96.3657 & 98.5803 & 99.9904 & -3.6247 & -1.4100 & $-3.6 \%$ & $-1.4 \%$ \\
\hline 6 & SB5VS-06 & $\mathrm{Al} 2 \mathrm{O} 3(\mathrm{wt} \%)$ & 8.1296 & 8.2395 & 8.1889 & -0.0593 & 0.0506 & $-0.7 \%$ & $0.6 \%$ \\
\hline 6 & SB5VS-06 & $\mathrm{B} 2 \mathrm{O} 3(\mathrm{wt} \%)$ & 5.7395 & 5.9914 & 5.2800 & 0.4595 & 0.7114 & $8.7 \%$ & $13.5 \%$ \\
\hline 6 & SB5VS-06 & $\mathrm{BaO}(\mathrm{wt} \%)$ & 0.0103 & 0.0100 & 0.0099 & 0.0004 & 0.0001 & $4.2 \%$ & $0.8 \%$ \\
\hline 6 & SB5VS-06 & $\mathrm{CaO}(\mathrm{wt} \%)$ & 1.0092 & 1.0149 & 1.0255 & -0.0164 & -0.0106 & $-1.6 \%$ & $-1.0 \%$ \\
\hline 6 & SB5VS-06 & $\mathrm{CdO}(\mathrm{wt} \%)$ & 0.0051 & 0.0051 & 0.0064 & -0.0013 & -0.0013 & $-19.7 \%$ & $-19.7 \%$ \\
\hline 6 & SB5VS-06 & $\mathrm{Ce} 2 \mathrm{O} 3(\mathrm{wt} \%)$ & 0.0243 & 0.0243 & 0.0027 & 0.0216 & 0.0216 & $787.3 \%$ & $787.3 \%$ \\
\hline 6 & SB5VS-06 & $\mathrm{Cr} 2 \mathrm{O} 3(\mathrm{wt} \%)$ & 0.0113 & 0.0111 & 0.0094 & 0.0019 & 0.0017 & $20.4 \%$ & $18.4 \%$ \\
\hline 6 & SB5VS-06 & $\mathrm{CuO}(\mathrm{wt} \%)$ & 0.0138 & 0.0137 & 0.0027 & 0.0110 & 0.0110 & $403.7 \%$ & $401.9 \%$ \\
\hline 6 & SB5VS-06 & $\mathrm{Fe} 2 \mathrm{O} 3(\mathrm{wt} \%)$ & 8.2637 & 8.2887 & 8.5992 & -0.3355 & -0.3105 & $-3.9 \%$ & $-3.6 \%$ \\
\hline 6 & SB5VS-06 & $\mathrm{K} 2 \mathrm{O}(\mathrm{wt} \%)$ & 0.0142 & 0.0139 & 0.0100 & 0.0042 & 0.0040 & $41.8 \%$ & $39.7 \%$ \\
\hline 6 & SB5VS-06 & $\mathrm{La} 2 \mathrm{O} 3(\mathrm{wt} \%)$ & 0.0001 & 0.0001 & 0.0020 & -0.0019 & -0.0019 & $-94.1 \%$ & $-94.1 \%$ \\
\hline 6 & SB5VS-06 & $\mathrm{Li} 2 \mathrm{O}(\mathrm{wt} \%)$ & 4.8171 & 5.3993 & 5.2810 & -0.4639 & 0.1183 & $-8.8 \%$ & $2.2 \%$ \\
\hline 6 & SB5VS-06 & $\mathrm{MgO}(\mathrm{wt} \%)$ & 0.3536 & 0.3408 & 0.3483 & 0.0053 & -0.0075 & $1.5 \%$ & $-2.2 \%$ \\
\hline 6 & SB5VS-06 & $\mathrm{MnO}\left(\mathrm{wt}^{2} \%\right)$ & 2.2273 & 2.2247 & 2.2344 & -0.0071 & -0.0096 & $-0.3 \%$ & $-0.4 \%$ \\
\hline 6 & SB5VS-06 & $\mathrm{Na} 2 \mathrm{O}(\mathrm{wt} \%)$ & 13.2542 & 13.0981 & 13.1625 & 0.0917 & -0.0645 & $0.7 \%$ & $-0.5 \%$ \\
\hline 6 & SB5VS-06 & $\mathrm{NiO}(\mathrm{wt} \%)$ & 1.2044 & 1.1834 & 1.2490 & -0.0446 & -0.0656 & $-3.6 \%$ & $-5.3 \%$ \\
\hline 6 & SB5VS-06 & $\mathrm{P} 2 \mathrm{O} 5(\mathrm{wt} \%)$ & 0.0206 & 0.0206 & 0.0290 & -0.0083 & -0.0083 & $-28.8 \%$ & $-28.8 \%$ \\
\hline 6 & SB5VS-06 & $\mathrm{PbO}(\mathrm{wt} \%)$ & 0.0102 & 0.0102 & 0.0022 & 0.0080 & 0.0080 & $359.5 \%$ & $359.5 \%$ \\
\hline 6 & SB5VS-06 & $\mathrm{SiO} 2(\mathrm{wt} \%)$ & 51.6641 & 51.3519 & 51.1540 & 0.5101 & 0.1979 & $1.0 \%$ & $0.4 \%$ \\
\hline 6 & SB5VS-06 & SO4 (wt\%) & 0.3940 & 0.3940 & 0.2913 & 0.1026 & 0.1026 & $35.2 \%$ & $35.2 \%$ \\
\hline 6 & SB5VS-06 & $\mathrm{TiO} 2(\mathrm{wt} \%)$ & 0.0150 & 0.0147 & 0.0000 & 0.0150 & 0.0147 & & \\
\hline 6 & SB5VS-06 & U3O8 (wt\%) & 2.9716 & 3.0468 & 3.0900 & -0.1185 & -0.0432 & $-3.8 \%$ & $-1.4 \%$ \\
\hline 6 & SB5VS-06 & $\mathrm{ZnO}(\mathrm{wt} \%)$ & 0.0084 & 0.0084 & 0.0008 & 0.0076 & 0.0076 & $902.7 \%$ & $902.7 \%$ \\
\hline 6 & SB5VS-06 & $\mathrm{ZrO} 2(\mathrm{wt} \%)$ & 0.0155 & 0.0155 & 0.0107 & 0.0049 & 0.0049 & $45.7 \%$ & $45.7 \%$ \\
\hline 6 & SB5VS-06 & Sum & 100.1772 & 100.7215 & 99.9901 & 0.1871 & 0.7314 & $0.2 \%$ & $0.7 \%$ \\
\hline
\end{tabular}


Table 5 cont. Summary of the Target, Average Measured/Bias-Corrected Chemical Compositions

\begin{tabular}{|c|c|c|c|c|c|c|c|c|c|}
\hline & & & & Measured & & & & & \\
\hline & Glass & & Measured & Bias-Corrected & Targeted & Diff of & Diff of & $\%$ Diff of & $\%$ Diff of \\
\hline Glass \# & ID & Oxide & $(w t \%)$ & $(w t \%)$ & $(w t \%)$ & Measured & Meas BC & Measured & Meas BC \\
\hline 7 & SB5VS-07 & $\mathrm{Al} 2 \mathrm{O} 3(\mathrm{wt} \%)$ & 6.1928 & 6.2482 & 6.4522 & -0.2594 & -0.2040 & $-4.0 \%$ & $-3.2 \%$ \\
\hline 7 & SB5VS-07 & $\mathrm{B} 2 \mathrm{O} 3(\mathrm{wt} \%)$ & 5.6187 & 5.8662 & 5.2800 & 0.3387 & 0.5862 & $6.4 \%$ & $11.1 \%$ \\
\hline 7 & SB5VS-07 & $\mathrm{BaO}(\mathrm{wt} \%)$ & 0.0435 & 0.0414 & 0.0448 & -0.0012 & -0.0034 & $-2.8 \%$ & $-7.5 \%$ \\
\hline 7 & SB5VS-07 & $\mathrm{CaO}(\mathrm{wt} \%)$ & 0.9420 & 0.9537 & 1.0255 & -0.0835 & -0.0718 & $-8.1 \%$ & $-7.0 \%$ \\
\hline 7 & SB5VS-07 & $\mathrm{CdO}(\mathrm{wt} \%)$ & 0.0266 & 0.0266 & 0.0289 & -0.0024 & -0.0024 & $-8.2 \%$ & $-8.2 \%$ \\
\hline 7 & SB5VS-07 & $\mathrm{Ce} 2 \mathrm{O} 3(\mathrm{wt} \%)$ & 0.0287 & 0.0287 & 0.0124 & 0.0163 & 0.0163 & $131.9 \%$ & $131.9 \%$ \\
\hline 7 & SB5VS-07 & $\mathrm{Cr} 2 \mathrm{O} 3(\mathrm{wt} \%)$ & 0.0387 & 0.0373 & 0.0425 & -0.0037 & -0.0052 & $-8.8 \%$ & $-12.2 \%$ \\
\hline 7 & SB5VS-07 & $\mathrm{CuO}(\mathrm{wt} \%)$ & 0.0200 & 0.0199 & 0.0123 & 0.0077 & 0.0075 & $62.2 \%$ & $60.9 \%$ \\
\hline 7 & SB5VS-07 & $\mathrm{Fe} 2 \mathrm{O} 3(\mathrm{wt} \%)$ & 8.3423 & 8.2204 & 9.0085 & -0.6662 & -0.7881 & $-7.4 \%$ & $-8.7 \%$ \\
\hline 7 & SB5VS-07 & $\mathrm{K} 2 \mathrm{O}(\mathrm{wt} \%)$ & 0.0428 & 0.0433 & 0.0451 & -0.0023 & -0.0018 & $-5.2 \%$ & $-4.0 \%$ \\
\hline 7 & SB5VS-07 & $\mathrm{La} 2 \mathrm{O} 3(\mathrm{wt} \%)$ & 0.0049 & 0.0049 & 0.0089 & -0.0040 & -0.0040 & $-45.2 \%$ & $-45.2 \%$ \\
\hline 7 & SB5VS-07 & $\mathrm{Li} 2 \mathrm{O}(\mathrm{wt} \%)$ & 4.6933 & 5.2629 & 5.2840 & -0.5907 & -0.0211 & $-11.2 \%$ & $-0.4 \%$ \\
\hline 7 & SB5VS-07 & $\mathrm{MgO}\left(\mathrm{wt}^{2} \%\right)$ & 0.6849 & 0.6938 & 0.7559 & -0.0710 & -0.0621 & $-9.4 \%$ & $-8.2 \%$ \\
\hline 7 & SB5VS-07 & $\mathrm{MnO}(\mathrm{wt} \%)$ & 1.7173 & 1.6614 & 1.8016 & -0.0843 & -0.1402 & $-4.7 \%$ & $-7.8 \%$ \\
\hline 7 & SB5VS-07 & $\mathrm{Na} 2 \mathrm{O}$ (wt\%) & 14.3899 & 14.0820 & 15.2232 & -0.8333 & -1.1412 & $-5.5 \%$ & $-7.5 \%$ \\
\hline 7 & SB5VS-07 & $\mathrm{NiO}(\mathrm{wt} \%)$ & 0.7470 & 0.7281 & 0.8081 & -0.0611 & -0.0801 & $-7.6 \%$ & $-9.9 \%$ \\
\hline 7 & SB5VS-07 & P2O5 (wt\%) & 0.0510 & 0.0510 & 0.1308 & -0.0798 & -0.0798 & $-61.0 \%$ & $-61.0 \%$ \\
\hline 7 & SB5VS-07 & $\mathrm{PbO}(\mathrm{wt} \%)$ & 0.0162 & 0.0162 & 0.0101 & 0.0061 & 0.0061 & $60.7 \%$ & $60.7 \%$ \\
\hline 7 & SB5VS-07 & $\mathrm{SiO} 2(\mathrm{wt} \%)$ & 50.7549 & 50.4687 & 51.1540 & -0.3991 & -0.6853 & $-0.8 \%$ & $-1.3 \%$ \\
\hline 7 & SB5VS-07 & $\mathrm{SO} 4(\mathrm{wt} \%)$ & 0.3850 & 0.3850 & 0.2913 & 0.0936 & 0.0936 & $32.1 \%$ & $32.1 \%$ \\
\hline 7 & SB5VS-07 & $\mathrm{TiO} 2(\mathrm{wt} \%)$ & 0.0096 & 0.0090 & 0.0000 & 0.0096 & 0.0090 & & \\
\hline 7 & SB5VS-07 & U3O8 (wt\%) & 2.2582 & 2.3794 & 2.4828 & -0.2247 & -0.1035 & $-9.0 \%$ & $-4.2 \%$ \\
\hline 7 & SB5VS-07 & $\mathrm{ZnO}(\mathrm{wt} \%)$ & 0.0115 & 0.0115 & 0.0038 & 0.0077 & 0.0077 & $204.1 \%$ & $204.1 \%$ \\
\hline 7 & SB5VS-07 & $\mathrm{ZrO} 2(\mathrm{wt} \%)$ & 0.0493 & 0.0493 & 0.0482 & 0.0011 & 0.0011 & $2.4 \%$ & $2.4 \%$ \\
\hline 7 & SB5VS-07 & Sum & 97.0690 & 97.2887 & 99.9549 & -2.8859 & -2.6662 & $-2.9 \%$ & $-2.7 \%$ \\
\hline 8 & SB5VS-08 & $\mathrm{Al} 2 \mathrm{O} 3(\mathrm{wt} \%)$ & 7.9548 & 8.0253 & 8.1889 & -0.2341 & -0.1636 & $-2.9 \%$ & $-2.0 \%$ \\
\hline 8 & SB5VS-08 & $\mathrm{B} 2 \mathrm{O} 3(\mathrm{wt} \%)$ & 5.2243 & 5.4266 & 5.2800 & -0.0557 & 0.1466 & $-1.1 \%$ & $2.8 \%$ \\
\hline 8 & SB5VS-08 & $\mathrm{BaO}(\mathrm{wt} \%)$ & 0.0466 & 0.0443 & 0.0448 & 0.0018 & -0.0004 & $4.1 \%$ & $-1.0 \%$ \\
\hline 8 & SB5VS-08 & $\mathrm{CaO}(\mathrm{wt} \%)$ & 0.5849 & 0.5921 & 0.6256 & -0.0408 & -0.0336 & $-6.5 \%$ & $-5.4 \%$ \\
\hline 8 & SB5VS-08 & $\mathrm{CdO}(\mathrm{wt} \%)$ & 0.0291 & 0.0291 & 0.0289 & 0.0002 & 0.0002 & $0.7 \%$ & $0.7 \%$ \\
\hline 8 & SB5VS-08 & $\mathrm{Ce} 2 \mathrm{O} 3(\mathrm{wt} \%)$ & 0.0278 & 0.0278 & 0.0124 & 0.0154 & 0.0154 & $124.8 \%$ & $124.8 \%$ \\
\hline 8 & SB5VS-08 & $\mathrm{Cr} 2 \mathrm{O} 3(\mathrm{wt} \%)$ & 0.0398 & 0.0384 & 0.0425 & -0.0026 & -0.0041 & $-6.2 \%$ & $-9.7 \%$ \\
\hline 8 & SB5VS-08 & $\mathrm{CuO}(\mathrm{wt} \%)$ & 0.0219 & 0.0217 & 0.0123 & 0.0096 & 0.0094 & $77.5 \%$ & $76.0 \%$ \\
\hline 8 & SB5VS-08 & $\mathrm{Fe} 2 \mathrm{O} 3(\mathrm{wt} \%)$ & 10.5977 & 10.4428 & 11.0159 & -0.4183 & -0.5731 & $-3.8 \%$ & $-5.2 \%$ \\
\hline 8 & SB5VS-08 & $\mathrm{K} 2 \mathrm{O}(\mathrm{wt} \%)$ & 0.0446 & 0.0451 & 0.0451 & -0.0005 & 0.0000 & $-1.2 \%$ & $0.1 \%$ \\
\hline 8 & SB5VS-08 & $\mathrm{La} 2 \mathrm{O} 3(\mathrm{wt} \%)$ & 0.0050 & 0.0050 & 0.0089 & -0.0040 & -0.0040 & $-44.5 \%$ & $-44.5 \%$ \\
\hline 8 & SB5VS-08 & $\mathrm{Li} 2 \mathrm{O}(\mathrm{wt} \%)$ & 4.8279 & 5.3022 & 5.2840 & -0.4561 & 0.0182 & $-8.6 \%$ & $0.3 \%$ \\
\hline 8 & SB5VS-08 & $\mathrm{MgO}(\mathrm{wt} \%)$ & 0.3026 & 0.3066 & 0.3483 & -0.0457 & -0.0417 & $-13.1 \%$ & $-12.0 \%$ \\
\hline 8 & SB5VS-08 & $\mathrm{MnO}\left(\mathrm{wt}^{2} \%\right)$ & 1.8141 & 1.7552 & 1.8016 & 0.0125 & -0.0464 & $0.7 \%$ & $-2.6 \%$ \\
\hline 8 & SB5VS-08 & $\mathrm{Na} 2 \mathrm{O}(\mathrm{wt} \%)$ & 11.8354 & 11.5824 & 12.0079 & -0.1724 & -0.4254 & $-1.4 \%$ & $-3.5 \%$ \\
\hline 8 & SB5VS-08 & $\mathrm{NiO}(\mathrm{wt} \%)$ & 1.1974 & 1.1671 & 1.2490 & -0.0516 & -0.0819 & $-4.1 \%$ & $-6.6 \%$ \\
\hline 8 & SB5VS-08 & $\mathrm{P} 2 \mathrm{O} 5(\mathrm{wt} \%)$ & 0.0390 & 0.0390 & 0.1308 & -0.0918 & -0.0918 & $-70.2 \%$ & $-70.2 \%$ \\
\hline 8 & SB5VS-08 & $\mathrm{PbO}(\mathrm{wt} \%)$ & 0.0180 & 0.0180 & 0.0101 & 0.0080 & 0.0080 & $79.4 \%$ & $79.4 \%$ \\
\hline 8 & SB5VS-08 & $\mathrm{SiO} 2(\mathrm{wt} \%)$ & 50.5410 & 51.4335 & 51.1540 & -0.6130 & 0.2795 & $-1.2 \%$ & $0.5 \%$ \\
\hline 8 & SB5VS-08 & SO4 (wt $\%)$ & 0.2150 & 0.2150 & 0.1293 & 0.0857 & 0.0857 & $66.3 \%$ & $66.3 \%$ \\
\hline 8 & SB5VS-08 & $\mathrm{TiO} 2(\mathrm{wt} \%)$ & 0.0092 & 0.0086 & 0.0000 & 0.0092 & 0.0086 & & \\
\hline 8 & SB5VS-08 & U3O8 (wt\%) & 2.2906 & 2.4133 & 2.4828 & -0.1922 & -0.0695 & $-7.7 \%$ & $-2.8 \%$ \\
\hline 8 & SB5VS-08 & $\mathrm{ZnO}(\mathrm{wt} \%)$ & 0.0096 & 0.0096 & 0.0038 & 0.0059 & 0.0059 & $154.8 \%$ & $154.8 \%$ \\
\hline 8 & SB5VS-08 & $\mathrm{ZrO} 2(\mathrm{wt} \%)$ & 0.0520 & 0.0520 & 0.0482 & 0.0038 & 0.0038 & $8.0 \%$ & $8.0 \%$ \\
\hline 8 & SB5VS-08 & Sum & 97.7283 & 99.0007 & 99.9549 & -2.2267 & $\begin{array}{l}-0.9543 \\
\end{array}$ & $-2.2 \%$ & $-1.0 \%$ \\
\hline
\end{tabular}


Table 5 cont. Summary of the Target, Average Measured/Bias-Corrected Chemical Compositions

\begin{tabular}{|c|c|c|c|c|c|c|c|c|c|}
\hline & & & & Measured & & & & & \\
\hline & Glass & & Measured & Bias-Corrected & Targeted & Diff of & Diff of & $\%$ Diff of & $\%$ Diff of \\
\hline Glass \# & ID & Oxide & $(w t \%)$ & $(w t \%)$ & $(w t \%)$ & Measured & Meas BC & Measured & Meas BC \\
\hline 9 & SB5VS-09 & $\mathrm{Al} 2 \mathrm{O} 3(\mathrm{wt} \%)$ & 7.5816 & 7.6841 & 8.1889 & -0.6072 & -0.5047 & $-7.4 \%$ & $-6.2 \%$ \\
\hline 9 & SB5VS-09 & $\mathrm{B} 2 \mathrm{O} 3(\mathrm{wt} \%)$ & 5.4738 & 5.6883 & 5.2800 & 0.1938 & 0.4083 & $3.7 \%$ & $7.7 \%$ \\
\hline 9 & SB5VS-09 & $\mathrm{BaO}(\mathrm{wt} \%)$ & 0.0441 & 0.0427 & 0.0448 & -0.0007 & -0.0021 & $-1.5 \%$ & $-4.6 \%$ \\
\hline 9 & SB5VS-09 & $\mathrm{CaO}(\mathrm{wt} \%)$ & 0.5674 & 0.5706 & 0.6256 & -0.0582 & -0.0550 & $-9.3 \%$ & $-8.8 \%$ \\
\hline 9 & 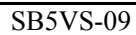 & $\mathrm{CdO}(\mathrm{wt} \%)$ & 0.0223 & 0.0223 & 0.0289 & -0.0067 & -0.0067 & $-23.0 \%$ & $-23.0 \%$ \\
\hline 9 & SB5VS-09 & $\mathrm{Ce} 2 \mathrm{O} 3(\mathrm{wt} \%)$ & 0.0246 & 0.0246 & 0.0124 & 0.0122 & 0.0122 & $98.8 \%$ & $98.8 \%$ \\
\hline 9 & SB5VS-09 & $\mathrm{Cr} 2 \mathrm{O} 3(\mathrm{wt} \%)$ & 0.0336 & 0.0330 & 0.0425 & -0.0089 & -0.0094 & $-20.9 \%$ & $-22.2 \%$ \\
\hline 9 & SB5VS-09 & $\mathrm{CuO}(\mathrm{wt} \%)$ & 0.0182 & 0.0181 & 0.0123 & 0.0058 & 0.0057 & $47.0 \%$ & $46.5 \%$ \\
\hline 9 & SB5VS-09 & $\mathrm{Fe} 2 \mathrm{O} 3(\mathrm{wt} \%)$ & 7.5595 & 7.5825 & 8.5992 & -1.0397 & -1.0167 & $-12.1 \%$ & $-11.8 \%$ \\
\hline 9 & SB5VS-09 & $\mathrm{K} 2 \mathrm{O}(\mathrm{wt} \%)$ & 0.0473 & 0.0466 & 0.0451 & 0.0022 & 0.0015 & $4.8 \%$ & $3.3 \%$ \\
\hline 9 & SB5VS-09 & $\mathrm{La} 2 \mathrm{O} 3(\mathrm{wt} \%)$ & 0.0027 & 0.0027 & 0.0089 & -0.0062 & -0.0062 & $-69.8 \%$ & $-69.8 \%$ \\
\hline 9 & SB5VS-09 & $\mathrm{Li} 2 \mathrm{O}(\mathrm{wt} \%)$ & 4.8171 & 5.2911 & 5.2840 & -0.4669 & 0.0071 & $-8.8 \%$ & $0.1 \%$ \\
\hline 9 & SB5VS-09 & $\mathrm{MgO}\left(\mathrm{wt}^{2} \%\right)$ & 0.7139 & 0.6880 & 0.7559 & -0.0420 & -0.0679 & $-5.6 \%$ & $-9.0 \%$ \\
\hline 9 & SB5VS-09 & $\mathrm{MnO}(\mathrm{wt} \%)$ & 2.0595 & 2.0571 & 2.2344 & -0.1749 & -0.1773 & $-7.8 \%$ & $-7.9 \%$ \\
\hline 9 & SB5VS-09 & $\mathrm{Na} 2 \mathrm{O}(\mathrm{wt} \%)$ & 12.1590 & 12.0157 & 12.8015 & -0.6425 & -0.7858 & $-5.0 \%$ & $-6.1 \%$ \\
\hline 9 & SB5VS-09 & $\mathrm{NiO}(\mathrm{wt} \%)$ & 0.7056 & 0.6933 & 0.8081 & -0.1025 & -0.1148 & $-12.7 \%$ & $-14.2 \%$ \\
\hline 9 & SB5VS-09 & P2O5 (wt\%) & 0.0504 & 0.0504 & 0.1308 & -0.0803 & -0.0803 & $-61.4 \%$ & $-61.4 \%$ \\
\hline 9 & SB5VS-09 & $\mathrm{PbO}(\mathrm{wt} \%)$ & 0.0108 & 0.0108 & 0.0101 & 0.0007 & 0.0007 & $7.1 \%$ & $7.1 \%$ \\
\hline 9 & SB5VS-09 & $\mathrm{SiO} 2(\mathrm{wt} \%)$ & 50.5944 & 51.4957 & 51.1540 & -0.5596 & 0.3417 & $-1.1 \%$ & $0.7 \%$ \\
\hline 9 & SB5VS-09 & $\mathrm{SO} 4(\mathrm{wt} \%)$ & 0.1535 & 0.1535 & 0.1293 & 0.0243 & 0.0243 & $18.8 \%$ & $18.8 \%$ \\
\hline 9 & SB5VS-09 & $\mathrm{TiO} 2(\mathrm{wt} \%)$ & 0.5771 & 0.5663 & 0.6164 & -0.0393 & -0.0501 & $-6.4 \%$ & $-8.1 \%$ \\
\hline 9 & SB5VS-09 & U3O8 (wt\%) & 2.7092 & 2.7777 & 3.0900 & -0.3808 & -0.3123 & $-12.3 \%$ & $-10.1 \%$ \\
\hline 9 & SB5VS-09 & $\mathrm{ZnO}(\mathrm{wt} \%)$ & 0.0112 & 0.0112 & 0.0038 & 0.0074 & 0.0074 & $195.9 \%$ & $195.9 \%$ \\
\hline 9 & SB5VS-09 & $\mathrm{ZrO} 2(\mathrm{wt} \%)$ & 0.0473 & 0.0473 & 0.0482 & -0.0009 & -0.0009 & $-1.8 \%$ & $-1.8 \%$ \\
\hline 9 & $\begin{array}{l}\text { SB5VS-09 } \\
\text { SBV }\end{array}$ & Sum & 95.9841 & 97.5735 & 99.9549 & -3.9708 & -2.3814 & $-4.0 \%$ & $-2.4 \%$ \\
\hline 10 & SB5VS-10 & $\mathrm{Al} 2 \mathrm{O} 3(\mathrm{wt} \%)$ & 7.9548 & 8.0258 & 8.1889 & -0.2341 & -0.1630 & $-2.9 \%$ & $-2.0 \%$ \\
\hline 10 & SB5VS-10 & $\mathrm{B} 2 \mathrm{O} 3(\mathrm{wt} \%)$ & 5.2323 & 5.4644 & 5.2800 & -0.0477 & 0.1844 & $-0.9 \%$ & $3.5 \%$ \\
\hline 10 & SB5VS-10 & $\mathrm{BaO}(\mathrm{wt} \%)$ & 0.0444 & 0.0422 & 0.0448 & -0.0004 & -0.0026 & $-0.9 \%$ & $-5.7 \%$ \\
\hline 10 & SB5VS-10 & $\mathrm{CaO}(\mathrm{wt} \%)$ & 0.9647 & 0.9767 & 1.0255 & -0.0608 & -0.0489 & $-5.9 \%$ & $-4.8 \%$ \\
\hline 10 & SB5VS-10 & $\mathrm{CdO}(\mathrm{wt} \%)$ & 0.0268 & 0.0268 & 0.0289 & -0.0021 & -0.0021 & $-7.2 \%$ & $-7.2 \%$ \\
\hline 10 & SB5VS-10 & $\mathrm{Ce} 2 \mathrm{O} 3(\mathrm{wt} \%)$ & 0.0346 & 0.0346 & 0.0124 & 0.0222 & 0.0222 & $179.3 \%$ & $179.3 \%$ \\
\hline 10 & SB5VS-10 & $\mathrm{Cr} 2 \mathrm{O} 3(\mathrm{wt} \%)$ & 0.0391 & 0.0377 & 0.0425 & -0.0034 & -0.0048 & $-7.9 \%$ & $-11.3 \%$ \\
\hline 10 & SB5VS-10 & $\mathrm{CuO}(\mathrm{wt} \%)$ & 0.0238 & 0.0236 & 0.0123 & 0.0114 & 0.0112 & $92.7 \%$ & $91.1 \%$ \\
\hline 10 & SB5VS-10 & $\mathrm{Fe} 2 \mathrm{O} 3(\mathrm{wt} \%)$ & 9.3860 & 9.2489 & 10.1067 & -0.7207 & -0.8578 & $-7.1 \%$ & $-8.5 \%$ \\
\hline 10 & SB5VS-10 & $\mathrm{K} 2 \mathrm{O}(\mathrm{wt} \%)$ & 0.0443 & 0.0448 & 0.0451 & -0.0008 & -0.0003 & $-1.8 \%$ & $-0.6 \%$ \\
\hline 10 & SB5VS-10 & $\mathrm{La} 2 \mathrm{O} 3(\mathrm{wt} \%)$ & 0.0044 & 0.0044 & 0.0089 & -0.0045 & -0.0045 & $-50.8 \%$ & $-50.8 \%$ \\
\hline 10 & SB5VS-10 & $\mathrm{Li} 2 \mathrm{O}(\mathrm{wt} \%)$ & 4.7256 & 5.2980 & 5.2840 & -0.5584 & 0.0140 & $-10.6 \%$ & $0.3 \%$ \\
\hline 10 & SB5VS-10 & $\mathrm{MgO}(\mathrm{wt} \%)$ & 0.2985 & 0.3024 & 0.3483 & -0.0498 & -0.0459 & $-14.3 \%$ & $-13.2 \%$ \\
\hline 10 & SB5VS-10 & $\mathrm{MnO}\left(\mathrm{wt}^{2} \%\right)$ & 1.7851 & 1.7270 & 1.8016 & -0.0165 & -0.0746 & $-0.9 \%$ & $-4.1 \%$ \\
\hline 10 & SB5VS-10 & $\mathrm{Na} 2 \mathrm{O}(\mathrm{wt} \%)$ & 11.8793 & 11.6248 & 12.0056 & -0.1264 & -0.3808 & $-1.1 \%$ & $-3.2 \%$ \\
\hline 10 & SB5VS-10 & $\mathrm{NiO}(\mathrm{wt} \%)$ & 0.7765 & 0.7569 & 0.8081 & -0.0316 & -0.0512 & $-3.9 \%$ & $-6.3 \%$ \\
\hline 10 & SB5VS-10 & $\mathrm{P} 2 \mathrm{O} 5(\mathrm{wt} \%)$ & 0.0332 & 0.0332 & 0.1308 & -0.0975 & -0.0975 & $-74.6 \%$ & $-74.6 \%$ \\
\hline 10 & SB5VS-10 & $\mathrm{PbO}(\mathrm{wt} \%)$ & 0.0129 & 0.0129 & 0.0101 & 0.0029 & 0.0029 & $28.5 \%$ & $28.5 \%$ \\
\hline 10 & SB5VS-10 & $\mathrm{SiO} 2(\mathrm{wt} \%)$ & 50.1131 & 49.8235 & 50.7210 & -0.6079 & -0.8975 & $-1.2 \%$ & $-1.8 \%$ \\
\hline 10 & SB5VS-10 & SO4 (wt $\%)$ & 0.3078 & 0.3078 & 0.2913 & 0.0165 & 0.0165 & $5.7 \%$ & $5.7 \%$ \\
\hline 10 & SB5VS-10 & $\mathrm{TiO} 2(\mathrm{wt} \%)$ & 0.6259 & 0.5849 & 0.6164 & 0.0095 & -0.0315 & $1.5 \%$ & $-5.1 \%$ \\
\hline 10 & SB5VS-10 & U3O8 (wt\%) & 2.8890 & 3.0443 & 3.0900 & -0.2010 & -0.0458 & $-6.5 \%$ & $-1.5 \%$ \\
\hline 10 & SB5VS-10 & $\mathrm{ZnO}(\mathrm{wt} \%)$ & 0.0106 & 0.0106 & 0.0038 & 0.0068 & 0.0068 & $179.5 \%$ & $179.5 \%$ \\
\hline 10 & SB5VS-10 & $\mathrm{ZrO} 2(\mathrm{wt} \%)$ & 0.0432 & 0.0432 & 0.0482 & -0.0049 & -0.0049 & $-10.2 \%$ & $-10.2 \%$ \\
\hline 10 & SB5VS-10 & Sum & 97.2560 & 97.4995 & 99.9552 & -2.6992 & -2.4558 & $-2.7 \%$ & $-2.5 \%$ \\
\hline
\end{tabular}


Table 5 cont. Summary of the Target, Average Measured/Bias-Corrected Chemical Compositions

\begin{tabular}{|c|c|c|c|c|c|c|c|c|c|}
\hline & & & & Measured & & & & & \\
\hline & Glass & & Measured & Bias-Corrected & Targeted & Diff of & Diff of & $\%$ Diff of & $\%$ Diff of \\
\hline Glass \# & ID & Oxide & $(\mathrm{wt} \%)$ & $(w t \%)$ & $(\mathrm{wt} \%)$ & Measured & Meas BC & Measured & Meas BC \\
\hline 11 & SB5VS-11 & $\mathrm{Al} 2 \mathrm{O} 3(\mathrm{wt} \%)$ & 6.4574 & 6.5447 & 6.4522 & 0.0051 & 0.0924 & $0.1 \%$ & $1.4 \%$ \\
\hline 11 & SB5VS-11 & $\mathrm{B} 2 \mathrm{O} 3(\mathrm{wt} \%)$ & 5.2967 & 5.5029 & 5.2800 & 0.0167 & 0.2229 & $0.3 \%$ & $4.2 \%$ \\
\hline 11 & SB5VS-11 & $\mathrm{BaO}(\mathrm{wt} \%)$ & 0.0486 & 0.0470 & 0.0448 & 0.0038 & 0.0022 & $8.5 \%$ & $5.0 \%$ \\
\hline 11 & SB5VS-11 & $\mathrm{CaO}(\mathrm{wt} \%)$ & 0.6094 & 0.6128 & 0.6256 & -0.0163 & $\begin{array}{l}-0.0128 \\
\end{array}$ & $-2.6 \%$ & $-2.0 \%$ \\
\hline 11 & SB5VS-11 & $\mathrm{CdO}(\mathrm{wt} \%)$ & 0.0283 & 0.0283 & 0.0289 & -0.0007 & -0.0007 & $-2.3 \%$ & $-2.3 \%$ \\
\hline 11 & SB5VS-11 & $\mathrm{Ce} 2 \mathrm{O} 3(\mathrm{wt} \%)$ & 0.0363 & 0.0363 & 0.0124 & 0.0239 & 0.0239 & $193.5 \%$ & $193.5 \%$ \\
\hline 11 & SB5VS-11 & $\mathrm{Cr} 2 \mathrm{O} 3(\mathrm{wt} \%)$ & 0.0347 & 0.0341 & 0.0425 & -0.0078 & -0.0084 & $-18.3 \%$ & $-19.7 \%$ \\
\hline 11 & SB5VS-11 & $\mathrm{CuO}\left(\mathrm{wt}^{2} \%\right)$ & 0.0213 & 0.0212 & 0.0123 & 0.0089 & 0.0089 & $72.4 \%$ & $71.8 \%$ \\
\hline 11 & SB5VS-11 & $\mathrm{Fe} 2 \mathrm{O} 3(\mathrm{wt} \%)$ & 10.3081 & 10.3389 & 11.0159 & -0.7078 & -0.6770 & $-6.4 \%$ & $-6.1 \%$ \\
\hline 11 & SB5VS-11 & $\mathrm{K} 2 \mathrm{O}(\mathrm{wt} \%)$ & 0.0452 & 0.0445 & 0.0451 & 0.0001 & -0.0006 & $0.2 \%$ & $-1.3 \%$ \\
\hline 11 & SB5VS-11 & $\mathrm{La} 2 \mathrm{O} 3(\mathrm{wt} \%)$ & 0.0072 & 0.0072 & 0.0089 & -0.0017 & -0.0017 & $-18.9 \%$ & $-18.9 \%$ \\
\hline 11 & SB5VS-11 & Li2O (wt\%) & 5.1078 & 5.6091 & 5.2840 & -0.1762 & 0.3251 & $-3.3 \%$ & $6.2 \%$ \\
\hline 11 & SB5VS-11 & $\mathrm{MgO}(\mathrm{wt} \%)$ & 0.8030 & 0.7742 & 0.7559 & 0.0471 & 0.0182 & $6.2 \%$ & $2.4 \%$ \\
\hline 11 & SB5VS-11 & $\mathrm{MnO}(\mathrm{wt} \%)$ & 2.2015 & 2.1989 & 2.2344 & -0.0329 & -0.0355 & $-1.5 \%$ & $-1.6 \%$ \\
\hline 11 & SB5VS-11 & $\mathrm{Na} 2 \mathrm{O}(\mathrm{wt} \%)$ & 12.6038 & 12.4555 & 12.5681 & 0.0357 & -0.1125 & $0.3 \%$ & $-0.9 \%$ \\
\hline 11 & SB5VS-11 & $\mathrm{NiO}(\mathrm{wt} \%)$ & 1.2353 & 1.2137 & 1.2490 & -0.0137 & -0.0353 & $-1.1 \%$ & $-2.8 \%$ \\
\hline 11 & SB5VS-11 & P2O5 (wt\%) & 0.0309 & 0.0309 & 0.1308 & -0.0998 & -0.0998 & $-76.3 \%$ & $-76.3 \%$ \\
\hline 11 & SB5VS-11 & $\mathrm{PbO}(\mathrm{wt} \%)$ & 0.0234 & 0.0234 & 0.0101 & 0.0134 & 0.0134 & $133.0 \%$ & $133.0 \%$ \\
\hline 11 & SB5VS-11 & $\mathrm{SiO} 2(\mathrm{wt} \%)$ & 52.3059 & 53.2205 & 50.7210 & 1.5849 & 2.4995 & $3.1 \%$ & $4.9 \%$ \\
\hline 11 & SB5VS-11 & SO4 (wt $\%)$ & 0.3880 & 0.3880 & 0.2913 & 0.0966 & 0.0966 & $33.2 \%$ & $33.2 \%$ \\
\hline 11 & SB5VS-11 & TiO2 (wt\%) & 0.0083 & 0.0082 & 0.0000 & 0.0083 & 0.0082 & & \\
\hline 11 & SB5VS-11 & U3O8 (wt\%) & 2.9274 & 3.0016 & 3.0900 & -0.1627 & -0.0885 & $-5.3 \%$ & $-2.9 \%$ \\
\hline 11 & SB5VS-11 & $\mathrm{ZnO}(\mathrm{wt} \%)$ & 0.0146 & 0.0146 & 0.0038 & 0.0108 & 0.0108 & $286.3 \%$ & $286.3 \%$ \\
\hline 11 & SB5VS-11 & $\mathrm{ZrO} 2(\mathrm{wt} \%)$ & 0.0459 & 0.0459 & 0.0482 & -0.0022 & -0.0022 & $-4.6 \%$ & $-4.6 \%$ \\
\hline 11 & SB5VS-11 & Sum & 100.5890 & 102.2024 & 99.9552 & 0.6338 & 2.2472 & $0.6 \%$ & $2.2 \%$ \\
\hline 12 & SB5VS-12 & $\mathrm{Al} 2 \mathrm{O} 3(\mathrm{wt} \%)$ & 6.4526 & 6.5399 & 6.4522 & 0.0004 & 0.0877 & $0.0 \%$ & $1.4 \%$ \\
\hline 12 & SB5VS-12 & $\mathrm{B} 2 \mathrm{O} 3(\mathrm{wt} \%)$ & 5.3209 & 5.5275 & 5.2800 & 0.0409 & 0.2475 & $0.8 \%$ & $4.7 \%$ \\
\hline 12 & SB5VS-12 & $\mathrm{BaO}(\mathrm{wt} \%)$ & 0.0114 & 0.0111 & 0.0099 & 0.0015 & 0.0012 & $15.4 \%$ & $11.7 \%$ \\
\hline 12 & SB5VS-12 & $\mathrm{CaO}(\mathrm{wt} \%)$ & 0.6122 & 0.6156 & 0.6256 & -0.0135 & -0.0100 & $-2.2 \%$ & $-1.6 \%$ \\
\hline 12 & SB5VS-12 & $\mathrm{CdO}(\mathrm{wt} \%)$ & 0.0046 & 0.0046 & 0.0064 & -0.0018 & -0.0018 & $-28.7 \%$ & $-28.7 \%$ \\
\hline 12 & SB5VS-12 & $\mathrm{Ce} 2 \mathrm{O} 3(\mathrm{wt} \%)$ & 0.0237 & 0.0237 & 0.0027 & 0.0210 & 0.0210 & $766.0 \%$ & $766.0 \%$ \\
\hline 12 & SB5VS-12 & $\mathrm{Cr} 2 \mathrm{O} 3(\mathrm{wt} \%)$ & 0.0088 & 0.0086 & 0.0094 & -0.0006 & -0.0008 & $-6.8 \%$ & $-8.4 \%$ \\
\hline 12 & SB5VS-12 & $\mathrm{CuO}(\mathrm{wt} \%)$ & 0.0153 & 0.0153 & 0.0027 & 0.0126 & 0.0125 & $460.9 \%$ & $459.0 \%$ \\
\hline 12 & SB5VS-12 & $\mathrm{Fe} 2 \mathrm{O} 3(\mathrm{wt} \%)$ & 9.9543 & 9.9844 & 10.1442 & -0.1899 & $\begin{array}{l}-0.1598 \\
\end{array}$ & $-1.9 \%$ & $-1.6 \%$ \\
\hline 12 & SB5VS-12 & $\mathrm{K} 2 \mathrm{O}$ (wt\%) & 0.0166 & 0.0163 & 0.0100 & 0.0066 & 0.0063 & $65.9 \%$ & $63.4 \%$ \\
\hline 12 & SB5VS-12 & $\mathrm{La} 2 \mathrm{O} 3(\mathrm{wt} \%)$ & 0.0001 & 0.0001 & 0.0020 & -0.0019 & -0.0019 & $-94.1 \%$ & $-94.1 \%$ \\
\hline 12 & SB5VS-12 & $\mathrm{Li} 2 \mathrm{O}(\mathrm{wt} \%)$ & 4.9947 & 5.4850 & 5.2810 & -0.2863 & 0.2040 & $-5.4 \%$ & $3.9 \%$ \\
\hline 12 & SB5VS-12 & $\mathrm{MgO}(\mathrm{wt} \%)$ & 0.3582 & 0.3453 & 0.3483 & 0.0099 & -0.0030 & $2.8 \%$ & $-0.9 \%$ \\
\hline 12 & SB5VS-12 & $\mathrm{MnO}(\mathrm{wt} \%)$ & 1.8109 & 1.8088 & 1.8016 & 0.0093 & 0.0072 & $0.5 \%$ & $0.4 \%$ \\
\hline 12 & SB5VS-12 & $\mathrm{Na} 2 \mathrm{O}(\mathrm{wt} \%)$ & 15.3672 & 15.1864 & 15.2232 & 0.1440 & -0.0368 & $0.9 \%$ & $-0.2 \%$ \\
\hline 12 & SB5VS-12 & $\mathrm{NiO}(\mathrm{wt} \%)$ & 0.7816 & 0.7680 & 0.8081 & -0.0265 & -0.0401 & $-3.3 \%$ & $-5.0 \%$ \\
\hline 12 & SB5VS-12 & P2O5 (wt\%) & 0.0206 & 0.0206 & 0.0290 & -0.0083 & -0.0083 & $-28.8 \%$ & $-28.8 \%$ \\
\hline 12 & SB5VS-12 & $\mathrm{PbO}(\mathrm{wt} \%)$ & 0.0113 & 0.0113 & 0.0022 & 0.0091 & 0.0091 & $407.9 \%$ & $407.9 \%$ \\
\hline 12 & SB5VS-12 & $\mathrm{SiO} 2(\mathrm{wt} \%)$ & 49.7922 & 50.6690 & 50.7210 & -0.9288 & -0.0520 & $-1.8 \%$ & $-0.1 \%$ \\
\hline 12 & SB5VS-12 & SO4 (wt $\%)$ & 0.2127 & 0.2127 & 0.1293 & 0.0835 & 0.0835 & $64.6 \%$ & $64.6 \%$ \\
\hline 12 & SB5VS-12 & $\mathrm{TiO} 2(\mathrm{wt} \%)$ & 0.0100 & 0.0098 & 0.0000 & 0.0100 & 0.0098 & & \\
\hline 12 & SB5VS-12 & U3O8 (wt\%) & 2.9303 & 3.0046 & 3.0900 & -0.1597 & -0.0854 & $-5.2 \%$ & $-2.8 \%$ \\
\hline 12 & SB5VS-12 & $\mathrm{ZnO}(\mathrm{wt} \%)$ & 0.0093 & 0.0093 & 0.0008 & 0.0085 & 0.0085 & \begin{tabular}{|l|}
$1014.1 \%$ \\
\end{tabular} & $1014.1 \%$ \\
\hline 12 & SB5VS-12 & $\mathrm{ZrO} 2(\mathrm{wt} \%)$ & 0.0169 & 0.0169 & 0.0107 & 0.0062 & 0.0062 & $58.3 \%$ & $58.3 \%$ \\
\hline 12 & SB5VS-12 & Sum & 98.7365 & 100.2949 & 99.9904 & -1.2539 & 0.3045 & $-1.3 \%$ & $0.3 \%$ \\
\hline
\end{tabular}


Table 5 cont. Summary of the Target, Average Measured/Bias-Corrected Chemical Compositions

\begin{tabular}{|c|c|c|c|c|c|c|c|c|c|}
\hline & & & & Measured & & & & & \\
\hline & Glass & & Measured & Bias-Corrected & Targeted & Diff of & Diff of & $\%$ Diff of & $\%$ Diff of \\
\hline Glass \# & ID & Oxide & $(w t \%)$ & $(w t \%)$ & $(w t \%)$ & Measured & Meas BC & Measured & Meas BC \\
\hline 13 & SB5VS-13 & $\mathrm{Al} 2 \mathrm{O} 3(\mathrm{wt} \%)$ & 7.0620 & 7.1246 & 7.2304 & -0.1684 & -0.1058 & $-2.3 \%$ & $-1.5 \%$ \\
\hline 13 & SB5VS-13 & $\mathrm{B} 2 \mathrm{O} 3(\mathrm{wt} \%)$ & 5.2726 & 5.4770 & 5.2800 & -0.0074 & 0.1970 & $-0.1 \%$ & $3.7 \%$ \\
\hline 13 & SB5VS-13 & $\mathrm{BaO}(\mathrm{wt} \%)$ & 0.0285 & 0.0271 & 0.0274 & 0.0011 & -0.0003 & $4.0 \%$ & $-1.0 \%$ \\
\hline 13 & SB5VS-13 & $\mathrm{CaO}(\mathrm{wt} \%)$ & 0.7783 & 0.7878 & 0.8246 & -0.0463 & -0.0368 & $-5.6 \%$ & $-4.5 \%$ \\
\hline 13 & SB5VS-13 & $\mathrm{CdO}(\mathrm{wt} \%)$ & 0.0183 & 0.0183 & 0.0177 & 0.0006 & 0.0006 & $3.4 \%$ & $3.4 \%$ \\
\hline 13 & SB5VS-13 & $\mathrm{Ce} 2 \mathrm{O} 3(\mathrm{wt} \%)$ & 0.0258 & 0.0258 & 0.0076 & 0.0182 & 0.0182 & $240.8 \%$ & $240.8 \%$ \\
\hline 13 & SB5VS-13 & $\mathrm{Cr} 2 \mathrm{O} 3(\mathrm{wt} \%)$ & 0.0248 & 0.0239 & 0.0260 & -0.0011 & -0.0020 & $-4.3 \%$ & $-7.8 \%$ \\
\hline 13 & SB5VS-13 & $\mathrm{CuO}(\mathrm{wt} \%)$ & 0.0163 & 0.0161 & 0.0075 & 0.0087 & 0.0086 & $115.7 \%$ & $113.9 \%$ \\
\hline 13 & SB5VS-13 & $\mathrm{Fe} 2 \mathrm{O} 3(\mathrm{wt} \%)$ & 9.3717 & 9.2348 & 9.7623 & -0.3906 & -0.5274 & $-4.0 \%$ & $-5.4 \%$ \\
\hline 13 & SB5VS-13 & $\mathrm{K} 2 \mathrm{O}(\mathrm{wt} \%)$ & 0.0289 & 0.0293 & 0.0276 & 0.0013 & 0.0017 & $4.9 \%$ & $6.2 \%$ \\
\hline 13 & SB5VS-13 & $\mathrm{La} 2 \mathrm{O} 3(\mathrm{wt} \%)$ & 0.0003 & 0.0003 & 0.0055 & -0.0052 & -0.0052 & $-94.6 \%$ & $-94.6 \%$ \\
\hline 13 & SB5VS-13 & $\mathrm{Li} 2 \mathrm{O}(\mathrm{wt} \%)$ & 4.9409 & 5.4248 & 5.2820 & -0.3411 & 0.1428 & $-6.5 \%$ & $2.7 \%$ \\
\hline 13 & SB5VS-13 & $\mathrm{MgO}(\mathrm{wt} \%)$ & 0.5062 & 0.5128 & 0.5511 & -0.0449 & -0.0383 & $-8.1 \%$ & $-6.9 \%$ \\
\hline 13 & SB5VS-13 & $\mathrm{MnO}(\mathrm{wt} \%)$ & 2.0110 & 1.9457 & 2.0163 & -0.0052 & -0.0706 & $-0.3 \%$ & $-3.5 \%$ \\
\hline 13 & SB5VS-13 & $\mathrm{Na} 2 \mathrm{O}(\mathrm{wt} \%)$ & 13.2980 & 13.0138 & 13.5243 & -0.2263 & -0.5106 & $-1.7 \%$ & $-3.8 \%$ \\
\hline 13 & SB5VS-13 & $\mathrm{NiO}(\mathrm{wt} \%)$ & 0.9770 & 0.9522 & 1.0266 & -0.0496 & -0.0743 & $-4.8 \%$ & $-7.2 \%$ \\
\hline 13 & SB5VS-13 & P2O5 (wt\%) & 0.0206 & 0.0206 & 0.0799 & -0.0593 & -0.0593 & $-74.2 \%$ & $-74.2 \%$ \\
\hline 13 & SB5VS-13 & $\mathrm{PbO}(\mathrm{wt} \%)$ & 0.0113 & 0.0113 & 0.0061 & 0.0052 & 0.0052 & $84.0 \%$ & $84.0 \%$ \\
\hline 13 & SB5VS-13 & $\mathrm{SiO} 2(\mathrm{wt} \%)$ & 51.7711 & 52.6716 & 50.9360 & 0.8351 & 1.7356 & $1.6 \%$ & $3.4 \%$ \\
\hline 13 & SB5VS-13 & SO4 (wt $\%)$ & 0.2869 & 0.2869 & 0.2105 & 0.0764 & 0.0764 & $36.3 \%$ & $36.3 \%$ \\
\hline 13 & SB5VS-13 & TiO2 (wt\%) & 0.3240 & 0.3028 & 0.3065 & 0.0175 & -0.0037 & $5.7 \%$ & $-1.2 \%$ \\
\hline 13 & SB5VS-13 & U3O8 (wt\%) & 2.5824 & 2.7208 & 2.7850 & -0.2026 & -0.0642 & $-7.3 \%$ & $-2.3 \%$ \\
\hline 13 & SB5VS-13 & $\mathrm{ZnO}(\mathrm{wt} \%)$ & 0.0106 & 0.0106 & 0.0023 & 0.0083 & 0.0083 & $357.3 \%$ & $357.3 \%$ \\
\hline 13 & SB5VS-13 & $\mathrm{ZrO} 2(\mathrm{wt} \%)$ & 0.0334 & 0.0334 & 0.0294 & 0.0040 & 0.0040 & $13.6 \%$ & $13.6 \%$ \\
\hline 13 & SB5VS-13 & $\begin{array}{l}\text { Sum } \\
\end{array}$ & 99.4009 & 100.6722 & 99.9724 & -0.5715 & 0.6999 & $-0.6 \%$ & $0.7 \%$ \\
\hline 14 & SB5VS-14 & $\mathrm{Al} 2 \mathrm{O} 3(\mathrm{wt} \%)$ & 7.3691 & 7.4444 & 7.4511 & -0.0821 & -0.0067 & $-1.1 \%$ & $-0.1 \%$ \\
\hline 14 & SB5VS-14 & $\mathrm{B} 2 \mathrm{O} 3(\mathrm{wt} \%)$ & 5.5060 & 5.4359 & 5.2800 & 0.2260 & 0.1559 & $4.3 \%$ & $3.0 \%$ \\
\hline 14 & SB5VS-14 & $\mathrm{BaO}(\mathrm{wt} \%)$ & 0.0475 & 0.0452 & 0.0483 & -0.0008 & -0.0030 & $-1.7 \%$ & $-6.3 \%$ \\
\hline 14 & SB5VS-14 & $\mathrm{CaO}(\mathrm{wt} \%)$ & 0.8213 & 0.8194 & 0.8381 & -0.0168 & -0.0187 & $-2.0 \%$ & $-2.2 \%$ \\
\hline 14 & SB5VS-14 & $\mathrm{CdO}(\mathrm{wt} \%)$ & 0.0308 & 0.0308 & 0.0333 & -0.0025 & -0.0025 & $-7.4 \%$ & $-7.4 \%$ \\
\hline 14 & SB5VS-14 & $\mathrm{Ce} 2 \mathrm{O} 3(\mathrm{wt} \%)$ & 0.0287 & 0.0287 & 0.0054 & 0.0233 & 0.0233 & $427.5 \%$ & $427.5 \%$ \\
\hline 14 & SB5VS-14 & $\mathrm{Cr} 2 \mathrm{O} 3(\mathrm{wt} \%)$ & 0.0343 & 0.0324 & 0.0347 & -0.0003 & -0.0022 & $-1.0 \%$ & $-6.5 \%$ \\
\hline 14 & SB5VS-14 & $\mathrm{CuO}(\mathrm{wt} \%)$ & 0.0178 & 0.0173 & 0.0085 & 0.0093 & 0.0088 & $109.9 \%$ & $103.4 \%$ \\
\hline 14 & SB5VS-14 & $\mathrm{Fe} 2 \mathrm{O} 3(\mathrm{wt} \%)$ & 9.4003 & 9.3295 & 10.0749 & -0.6746 & -0.7454 & $-6.7 \%$ & $-7.4 \%$ \\
\hline 14 & SB5VS-14 & $\mathrm{K} 2 \mathrm{O}$ (wt\%) & 0.0434 & 0.0432 & 0.0442 & -0.0008 & -0.0010 & $-1.9 \%$ & $-2.3 \%$ \\
\hline 14 & SB5VS-14 & $\mathrm{La} 2 \mathrm{O} 3(\mathrm{wt} \%)$ & 0.0001 & 0.0001 & 0.0041 & -0.0040 & -0.0040 & $-97.1 \%$ & $-97.1 \%$ \\
\hline 14 & SB5VS-14 & $\mathrm{Li} 2 \mathrm{O}(\mathrm{wt} \%)$ & 4.8978 & 5.3398 & 5.2800 & -0.3822 & 0.0598 & $-7.2 \%$ & $1.1 \%$ \\
\hline 14 & SB5VS-14 & $\mathrm{MgO}(\mathrm{wt} \%)$ & 0.5588 & 0.5455 & 0.5508 & 0.0080 & -0.0053 & $1.5 \%$ & $-1.0 \%$ \\
\hline 14 & SB5VS-14 & $\mathrm{MnO}(\mathrm{wt} \%)$ & 2.0466 & 1.9969 & 2.0461 & 0.0004 & -0.0492 & $0.0 \%$ & $-2.4 \%$ \\
\hline 14 & SB5VS-14 & $\mathrm{Na} 2 \mathrm{O}(\mathrm{wt} \%)$ & 12.9206 & 12.5945 & 13.0160 & -0.0954 & -0.4215 & $-0.7 \%$ & $-3.2 \%$ \\
\hline 14 & SB5VS-14 & $\mathrm{NiO}(\mathrm{wt} \%)$ & 1.0498 & 1.0044 & 1.0741 & -0.0242 & -0.0696 & $-2.3 \%$ & $-6.5 \%$ \\
\hline 14 & SB5VS-14 & P2O5 (wt\%) & 0.0544 & 0.0544 & 0.1511 & -0.0967 & -0.0967 & $-64.0 \%$ & $-64.0 \%$ \\
\hline 14 & SB5VS-14 & $\mathrm{PbO}(\mathrm{wt} \%)$ & 0.0105 & 0.0105 & 0.0044 & 0.0061 & 0.0061 & $137.6 \%$ & $137.6 \%$ \\
\hline 14 & SB5VS-14 & $\mathrm{SiO} 2(\mathrm{wt} \%)$ & 48.1877 & 50.3571 & 50.9170 & -2.7293 & -0.5599 & $-5.4 \%$ & $-1.1 \%$ \\
\hline 14 & SB5VS-14 & SO4 (wt $\%)$ & 0.3056 & 0.3056 & 0.2111 & 0.0945 & 0.0945 & $44.8 \%$ & $44.8 \%$ \\
\hline 14 & SB5VS-14 & $\mathrm{TiO} 2(\mathrm{wt} \%)$ & 0.0117 & 0.0114 & 0.0034 & 0.0083 & 0.0080 & $243.4 \%$ & $236.4 \%$ \\
\hline 14 & SB5VS-14 & U3O8 (wt\%) & 2.6974 & 2.7703 & 2.8319 & -0.1344 & -0.0615 & $-4.7 \%$ & $-2.2 \%$ \\
\hline 14 & SB5VS-14 & $\mathrm{ZnO}(\mathrm{wt} \%)$ & 0.0100 & 0.0100 & 0.0034 & 0.0066 & 0.0066 & $192.9 \%$ & $192.9 \%$ \\
\hline 14 & SB5VS-14 & $\mathrm{ZrO} 2(\mathrm{wt} \%)$ & 0.0554 & 0.0554 & 0.0503 & 0.0051 & 0.0051 & $10.1 \%$ & $10.1 \%$ \\
\hline 14 & SB5VS-14 & Sum & 96.1057 & 98.2828 & 99.9622 & -3.8565 & -1.6793 & $-3.9 \%$ & $-1.7 \%$ \\
\hline
\end{tabular}


Table 5 cont. Summary of the Target, Average Measured/Bias-Corrected Chemical Compositions

\begin{tabular}{|c|c|c|c|c|c|c|c|c|c|}
\hline & & & & Measured & & & & & \\
\hline & Glass & & Measured & Bias-Corrected & Targeted & Diff of & Diff of & $\%$ Diff of & $\%$ Diff of \\
\hline Glass \# & ID & Oxide & $(w t \%)$ & $(w t \%)$ & $(w t \%)$ & Measured & Meas BC & Measured & Meas BC \\
\hline 15 & SB5VS-15 & $\mathrm{Al} 2 \mathrm{O} 3(\mathrm{wt} \%)$ & 7.1423 & 7.2055 & 7.1832 & -0.0409 & 0.0223 & $-0.6 \%$ & $0.3 \%$ \\
\hline 15 & SB5VS-15 & $\mathrm{B} 2 \mathrm{O} 3(\mathrm{wt} \%)$ & 4.8057 & 5.0155 & 5.2800 & -0.4743 & -0.2645 & $-9.0 \%$ & $-5.0 \%$ \\
\hline 15 & SB5VS-15 & $\mathrm{BaO}(\mathrm{wt} \%)$ & 0.0491 & 0.0467 & 0.0473 & 0.0019 & -0.0005 & $3.9 \%$ & $-1.1 \%$ \\
\hline 15 & SB5VS-15 & $\mathrm{CaO}(\mathrm{wt} \%)$ & 0.7843 & 0.7939 & 0.8129 & -0.0287 & -0.0190 & $-3.5 \%$ & $-2.3 \%$ \\
\hline 15 & SB5VS-15 & $\mathrm{CdO}(\mathrm{wt} \%)$ & 0.0306 & 0.0306 & 0.0316 & -0.0011 & -0.0011 & $-3.4 \%$ & $-3.4 \%$ \\
\hline 15 & SB5VS-15 & $\mathrm{Ce} 2 \mathrm{O} 3(\mathrm{wt} \%)$ & 0.0305 & 0.0305 & 0.0082 & 0.0223 & 0.0223 & $273.2 \%$ & $273.2 \%$ \\
\hline 15 & SB5VS-15 & $\mathrm{Cr} 2 \mathrm{O} 3(\mathrm{wt} \%)$ & 0.0343 & 0.0331 & 0.0347 & -0.0003 & -0.0016 & $-1.0 \%$ & $-4.6 \%$ \\
\hline 15 & SB5VS-15 & $\mathrm{CuO}(\mathrm{wt} \%)$ & 0.0197 & 0.0196 & 0.0088 & 0.0109 & 0.0107 & $123.0 \%$ & $121.2 \%$ \\
\hline 15 & SB5VS-15 & $\mathrm{Fe} 2 \mathrm{O} 3(\mathrm{wt} \%)$ & 9.3538 & 9.2172 & 9.7757 & -0.4219 & -0.5585 & $-4.3 \%$ & $-5.7 \%$ \\
\hline 15 & SB5VS-15 & $\mathrm{K} 2 \mathrm{O}(\mathrm{wt} \%)$ & 0.0440 & 0.0445 & 0.0439 & 0.0001 & 0.0007 & $0.2 \%$ & $1.5 \%$ \\
\hline 15 & SB5VS-15 & $\mathrm{La} 2 \mathrm{O} 3(\mathrm{wt} \%)$ & 0.0013 & 0.0013 & 0.0051 & -0.0038 & -0.0038 & $-74.1 \%$ & $-74.1 \%$ \\
\hline 15 & SB5VS-15 & $\mathrm{Li} 2 \mathrm{O}(\mathrm{wt} \%)$ & 4.8333 & 5.4180 & 5.2800 & -0.4467 & 0.1380 & $-8.5 \%$ & $2.6 \%$ \\
\hline 15 & SB5VS-15 & $\mathrm{MgO}(\mathrm{wt} \%)$ & 0.4896 & 0.4960 & 0.5250 & -0.0353 & -0.0290 & $-6.7 \%$ & $-5.5 \%$ \\
\hline 15 & SB5VS-15 & $\mathrm{MnO}(\mathrm{wt} \%)$ & 2.0433 & 1.9769 & 2.0206 & 0.0227 & -0.0437 & $1.1 \%$ & $-2.2 \%$ \\
\hline 15 & SB5VS-15 & $\mathrm{Na} 2 \mathrm{O}(\mathrm{wt} \%)$ & 13.2205 & 12.9381 & 13.3026 & -0.0821 & -0.3646 & $-0.6 \%$ & $-2.7 \%$ \\
\hline 15 & SB5VS-15 & $\mathrm{NiO}(\mathrm{wt} \%)$ & 1.0260 & 1.0000 & 1.0431 & -0.0172 & $\begin{array}{l}-0.0431 \\
\end{array}$ & $-1.6 \%$ & $-4.1 \%$ \\
\hline 15 & SB5VS-15 & P2O5 (wt\%) & 0.0407 & 0.0407 & 0.1446 & -0.1039 & -0.1039 & $-71.9 \%$ & $-71.9 \%$ \\
\hline 15 & SB5VS-15 & $\mathrm{PbO}(\mathrm{wt} \%)$ & 0.0097 & 0.0097 & 0.0061 & 0.0036 & 0.0036 & $58.4 \%$ & $58.4 \%$ \\
\hline 15 & SB5VS-15 & $\mathrm{SiO} 2(\mathrm{wt} \%)$ & 51.1828 & 50.8784 & 50.8910 & 0.2918 & -0.0126 & $0.6 \%$ & $0.0 \%$ \\
\hline 15 & SB5VS-15 & SO4 (wt $\%)$ & 0.3063 & 0.3063 & 0.2490 & 0.0573 & 0.0573 & $23.0 \%$ & $23.0 \%$ \\
\hline 15 & SB5VS-15 & TiO2 (wt\%) & 0.4604 & 0.4302 & 0.4444 & 0.0160 & -0.0142 & $3.6 \%$ & $-3.2 \%$ \\
\hline 15 & SB5VS-15 & U3O8 (wt\%) & 2.5795 & 2.7176 & 2.7445 & -0.1650 & -0.0269 & $-6.0 \%$ & $-1.0 \%$ \\
\hline 15 & SB5VS-15 & $\mathrm{ZnO}(\mathrm{wt} \%)$ & 0.0112 & 0.0112 & 0.0048 & 0.0064 & 0.0064 & $135.4 \%$ & $135.4 \%$ \\
\hline 15 & SB5VS-15 & $\mathrm{ZrO} 2(\mathrm{wt} \%)$ & 0.0540 & 0.0540 & 0.0510 & 0.0030 & 0.0030 & $5.9 \%$ & $5.9 \%$ \\
\hline 15 & SB5VS-15 & Sum & 98.5528 & 98.7153 & 99.9380 & -1.3852 & -1.2227 & $-1.4 \%$ & $-1.2 \%$ \\
\hline 16 & SB5VS-16 & $\mathrm{Al} 2 \mathrm{O} 3(\mathrm{wt} \%)$ & 6.7455 & 6.8055 & 6.7937 & -0.0481 & 0.0118 & $-0.7 \%$ & $0.2 \%$ \\
\hline 16 & SB5VS-16 & $\mathrm{B} 2 \mathrm{O} 3(\mathrm{wt} \%)$ & 5.6268 & 5.8746 & 5.5200 & 0.1068 & 0.3546 & $1.9 \%$ & $6.4 \%$ \\
\hline 16 & SB5VS-16 & $\mathrm{BaO}(\mathrm{wt} \%)$ & 0.0447 & 0.0425 & 0.0440 & 0.0006 & -0.0015 & $1.5 \%$ & $-3.5 \%$ \\
\hline 16 & SB5VS-16 & $\mathrm{CaO}(\mathrm{wt} \%)$ & 0.7244 & 0.7333 & 0.7642 & -0.0397 & -0.0308 & $-5.2 \%$ & $-4.0 \%$ \\
\hline 16 & SB5VS-16 & $\mathrm{CdO}(\mathrm{wt} \%)$ & 0.0271 & 0.0271 & 0.0304 & -0.0033 & -0.0033 & $-10.7 \%$ & $-10.7 \%$ \\
\hline 16 & SB5VS-16 & $\mathrm{Ce} 2 \mathrm{O} 3(\mathrm{wt} \%)$ & 0.0258 & 0.0258 & 0.0050 & 0.0208 & 0.0208 & $419.5 \%$ & $419.5 \%$ \\
\hline 16 & SB5VS-16 & $\mathrm{Cr} 2 \mathrm{O} 3(\mathrm{wt} \%)$ & 0.0311 & 0.0299 & 0.0316 & -0.0006 & -0.0017 & $-1.8 \%$ & $-5.4 \%$ \\
\hline 16 & SB5VS-16 & $\mathrm{CuO}(\mathrm{wt} \%)$ & 0.0156 & 0.0155 & 0.0078 & 0.0079 & 0.0078 & $101.9 \%$ & $100.3 \%$ \\
\hline 16 & SB5VS-16 & $\mathrm{Fe} 2 \mathrm{O} 3(\mathrm{wt} \%)$ & 8.8570 & 8.7275 & 9.1859 & -0.3289 & -0.4584 & $-3.6 \%$ & $-5.0 \%$ \\
\hline 16 & SB5VS-16 & $\mathrm{K} 2 \mathrm{O}$ (wt\%) & 0.0373 & 0.0378 & 0.0403 & -0.0030 & -0.0025 & $-7.3 \%$ & $-6.2 \%$ \\
\hline 16 & SB5VS-16 & $\mathrm{La} 2 \mathrm{O} 3(\mathrm{wt} \%)$ & 0.0005 & 0.0005 & 0.0037 & -0.0033 & -0.0033 & $-87.4 \%$ & $-87.4 \%$ \\
\hline 16 & SB5VS-16 & Li2O (wt\%) & 4.9678 & 5.5670 & 5.5200 & -0.5522 & 0.0470 & $-10.0 \%$ & $0.9 \%$ \\
\hline 16 & SB5VS-16 & $\mathrm{MgO}(\mathrm{wt} \%)$ & 0.4386 & 0.4444 & 0.5022 & -0.0636 & -0.0578 & $-12.7 \%$ & $-11.5 \%$ \\
\hline 16 & SB5VS-16 & $\mathrm{MnO}(\mathrm{wt} \%)$ & 1.8916 & 1.8301 & 1.8656 & 0.0260 & -0.0355 & $1.4 \%$ & $-1.9 \%$ \\
\hline 16 & SB5VS-16 & $\mathrm{Na} 2 \mathrm{O}(\mathrm{wt} \%)$ & 12.5229 & 12.2551 & 12.5734 & -0.0505 & -0.3183 & $-0.4 \%$ & $-2.5 \%$ \\
\hline 16 & SB5VS-16 & $\mathrm{NiO}(\mathrm{wt} \%)$ & 0.9486 & 0.9246 & 0.9793 & -0.0306 & -0.0546 & $-3.1 \%$ & $-5.6 \%$ \\
\hline 16 & SB5VS-16 & P2O5 (wt\%) & 0.0481 & 0.0481 & 0.1378 & -0.0897 & -0.0897 & $-65.1 \%$ & $-65.1 \%$ \\
\hline 16 & SB5VS-16 & $\mathrm{PbO}(\mathrm{wt} \%)$ & 0.0121 & 0.0121 & 0.0040 & 0.0081 & 0.0081 & $200.7 \%$ & $200.7 \%$ \\
\hline 16 & SB5VS-16 & $\mathrm{SiO} 2(\mathrm{wt} \%)$ & 52.7337 & 52.4125 & 53.1300 & -0.3963 & -0.7175 & $-0.7 \%$ & $-1.4 \%$ \\
\hline 16 & SB5VS-16 & SO4 (wt $\%)$ & 0.2786 & 0.2786 & 0.1925 & 0.0861 & 0.0861 & $44.8 \%$ & $44.8 \%$ \\
\hline 16 & SB5VS-16 & $\mathrm{TiO} 2(\mathrm{wt} \%)$ & 0.0129 & 0.0121 & 0.0031 & 0.0098 & 0.0090 & $317.0 \%$ & $289.6 \%$ \\
\hline 16 & SB5VS-16 & U3O8 (wt\%) & 2.4144 & 2.5438 & 2.5820 & -0.1676 & -0.0382 & $-6.5 \%$ & $-1.5 \%$ \\
\hline 16 & SB5VS-16 & $\mathrm{ZnO}(\mathrm{wt} \%)$ & 0.0096 & 0.0096 & 0.0031 & 0.0065 & 0.0065 & $211.2 \%$ & $211.2 \%$ \\
\hline 16 & SB5VS-16 & $\mathrm{ZrO} 2(\mathrm{wt} \%)$ & 0.0439 & 0.0439 & 0.0459 & -0.0020 & -0.0020 & $-4.3 \%$ & $-4.3 \%$ \\
\hline 16 & SB5VS-16 & Sum & 98.4589 & 98.7020 & 99.9653 & -1.5064 & -1.2633 & $-1.5 \%$ & $-1.3 \%$ \\
\hline
\end{tabular}


Table 5 cont. Summary of the Target, Average Measured/Bias-Corrected Chemical Compositions

\begin{tabular}{|c|c|c|c|c|c|c|c|c|c|}
\hline & & & & Measured & & & & & \\
\hline & Glass & & Measured & Bias-Corrected & Targeted & Diff of & Diff of & $\%$ Diff of & $\%$ Diff of \\
\hline Glass \# & ID & Oxide & $(w t \%)$ & $(w t \%)$ & $(w t \%)$ & Measured & Meas BC & Measured & Meas BC \\
\hline 17 & SB5VS-17 & $\mathrm{Al} 2 \mathrm{O} 3(\mathrm{wt} \%)$ & 7.6619 & 7.7402 & 8.1086 & -0.4466 & -0.3684 & $-5.5 \%$ & $-4.5 \%$ \\
\hline 17 & SB5VS-17 & $\mathrm{B} 2 \mathrm{O} 3(\mathrm{wt} \%)$ & 5.3048 & 5.2375 & 5.0400 & 0.2648 & 0.1975 & $5.3 \%$ & $3.9 \%$ \\
\hline 17 & SB5VS-17 & $\mathrm{BaO}(\mathrm{wt} \%)$ & 0.0475 & 0.0452 & 0.0525 & $\begin{array}{l}-0.0051 \\
\end{array}$ & -0.0073 & $-9.7 \%$ & $-13.9 \%$ \\
\hline 17 & SB5VS-17 & $\mathrm{CaO}(\mathrm{wt} \%)$ & 0.8623 & 0.8602 & 0.9121 & -0.0498 & -0.0518 & $-5.5 \%$ & $-5.7 \%$ \\
\hline 17 & SB5VS-17 & $\mathrm{CdO}(\mathrm{wt} \%)$ & 0.0306 & 0.0306 & 0.0363 & -0.0057 & -0.0057 & $-15.7 \%$ & $-15.7 \%$ \\
\hline 17 & SB5VS-17 & $\mathrm{Ce} 2 \mathrm{O} 3(\mathrm{wt} \%)$ & 0.0284 & 0.0284 & 0.0059 & 0.0225 & 0.0225 & $379.8 \%$ & $379.8 \%$ \\
\hline 17 & SB5VS-17 & $\mathrm{Cr} 2 \mathrm{O} 3(\mathrm{wt} \%)$ & 0.0362 & 0.0342 & 0.0377 & -0.0016 & -0.0036 & $-4.1 \%$ & $-9.5 \%$ \\
\hline 17 & SB5VS-17 & $\mathrm{CuO}(\mathrm{wt} \%)$ & 0.0163 & 0.0158 & 0.0093 & 0.0070 & 0.0065 & $75.9 \%$ & $70.5 \%$ \\
\hline 17 & SB5VS-17 & $\mathrm{Fe} 2 \mathrm{O} 3(\mathrm{wt} \%)$ & 9.7970 & 9.7233 & 10.9638 & -1.1668 & -1.2405 & $-10.6 \%$ & $-11.3 \%$ \\
\hline 17 & SB5VS-17 & $\mathrm{K} 2 \mathrm{O}(\mathrm{wt} \%)$ & 0.0479 & 0.0477 & 0.0481 & -0.0002 & -0.0004 & $-0.5 \%$ & $-0.9 \%$ \\
\hline 17 & SB5VS-17 & $\mathrm{La} 2 \mathrm{O} 3(\mathrm{wt} \%)$ & 0.0003 & 0.0003 & 0.0044 & -0.0042 & -0.0042 & $-94.1 \%$ & $-94.1 \%$ \\
\hline 17 & SB5VS-17 & $\mathrm{Li} 2 \mathrm{O}(\mathrm{wt} \%)$ & 4.7633 & 5.1931 & 5.0400 & -0.2767 & 0.1531 & $-5.5 \%$ & $3.0 \%$ \\
\hline 17 & SB5VS-17 & $\mathrm{MgO}\left(\mathrm{wt}^{2} \%\right)$ & 0.5746 & 0.5608 & 0.5994 & -0.0248 & -0.0386 & $-4.1 \%$ & $-6.4 \%$ \\
\hline 17 & SB5VS-17 & $\mathrm{MnO}(\mathrm{wt} \%)$ & 2.1111 & 2.0601 & 2.2267 & -0.1155 & -0.1665 & $-5.2 \%$ & $-7.5 \%$ \\
\hline 17 & SB5VS-17 & $\mathrm{Na} 2 \mathrm{O}$ (wt\%) & 12.8835 & 12.5584 & 13.4586 & -0.5751 & -0.9002 & $-4.3 \%$ & $-6.7 \%$ \\
\hline 17 & SB5VS-17 & $\mathrm{NiO}(\mathrm{wt} \%)$ & 1.0724 & 1.0261 & 1.1688 & -0.0964 & -0.1428 & $-8.3 \%$ & $-12.2 \%$ \\
\hline 17 & SB5VS-17 & P2O5 (wt\%) & 0.0573 & 0.0573 & 0.1645 & -0.1072 & -0.1072 & $-65.2 \%$ & $-65.2 \%$ \\
\hline 17 & SB5VS-17 & $\mathrm{PbO}(\mathrm{wt} \%)$ & 0.0127 & 0.0127 & 0.0048 & 0.0078 & 0.0078 & $163.1 \%$ & $163.1 \%$ \\
\hline 17 & SB5VS-17 & $\mathrm{SiO} 2(\mathrm{wt} \%)$ & 47.7599 & 49.9100 & 48.7040 & -0.9441 & 1.2060 & $-1.9 \%$ & $2.5 \%$ \\
\hline 17 & SB5VS-17 & $\mathrm{SO} 4(\mathrm{wt} \%)$ & 0.3258 & 0.3258 & 0.2297 & 0.0961 & 0.0961 & $41.8 \%$ & $41.8 \%$ \\
\hline 17 & SB5VS-17 & $\mathrm{TiO} 2(\mathrm{wt} \%)$ & 0.0104 & 0.0102 & 0.0037 & 0.0067 & 0.0065 & $181.8 \%$ & $176.1 \%$ \\
\hline 17 & SB5VS-17 & U3O8 (wt\%) & 2.7800 & 2.8551 & 3.0817 & -0.3018 & -0.2266 & $-9.8 \%$ & $-7.4 \%$ \\
\hline 17 & SB5VS-17 & $\mathrm{ZnO}(\mathrm{wt} \%)$ & 0.0118 & 0.0118 & 0.0037 & 0.0081 & 0.0081 & $219.6 \%$ & $219.6 \%$ \\
\hline 17 & SB5VS-17 & $\mathrm{ZrO} 2(\mathrm{wt} \%)$ & 0.0530 & 0.0530 & 0.0548 & -0.0017 & -0.0017 & $-3.2 \%$ & $-3.2 \%$ \\
\hline 17 & SB5VS-17 & Sum & 96.2488 & 98.3977 & 99.9591 & -3.7103 & -1.5614 & $-3.7 \%$ & $-1.6 \%$ \\
\hline 18 & SB5VS-18 & $\mathrm{Al} 2 \mathrm{O} 3(\mathrm{wt} \%)$ & 5.0922 & 5.1376 & 5.2818 & -0.1895 & -0.1442 & $-3.6 \%$ & $-2.7 \%$ \\
\hline 18 & SB5VS-18 & $\mathrm{B} 2 \mathrm{O} 3(\mathrm{wt} \%)$ & 5.9971 & 6.2302 & 6.0000 & -0.0029 & 0.2302 & $0.0 \%$ & $3.8 \%$ \\
\hline 18 & SB5VS-18 & $\mathrm{BaO}(\mathrm{wt} \%)$ & 0.0324 & 0.0308 & 0.0348 & -0.0024 & -0.0039 & $-6.8 \%$ & $-11.4 \%$ \\
\hline 18 & SB5VS-18 & $\mathrm{CaO}(\mathrm{wt} \%)$ & 0.5443 & 0.5509 & 0.5978 & -0.0535 & -0.0468 & $-8.9 \%$ & $-7.8 \%$ \\
\hline 18 & SB5VS-18 & $\mathrm{CdO}(\mathrm{wt} \%)$ & 0.0220 & 0.0220 & 0.0233 & -0.0013 & -0.0013 & $-5.4 \%$ & $-5.4 \%$ \\
\hline 18 & SB5VS-18 & $\mathrm{Ce} 2 \mathrm{O} 3(\mathrm{wt} \%)$ & 0.0217 & 0.0217 & 0.0060 & 0.0157 & 0.0157 & $261.2 \%$ & $261.2 \%$ \\
\hline 18 & SB5VS-18 & $\mathrm{Cr} 2 \mathrm{O} 3(\mathrm{wt} \%)$ & 0.0263 & 0.0253 & 0.0255 & 0.0008 & -0.0002 & $3.2 \%$ & $-0.6 \%$ \\
\hline 18 & SB5VS-18 & $\mathrm{CuO}(\mathrm{wt} \%)$ & 0.0131 & 0.0130 & 0.0065 & 0.0066 & 0.0065 & $102.2 \%$ & $100.6 \%$ \\
\hline 18 & SB5VS-18 & $\mathrm{Fe} 2 \mathrm{O} 3(\mathrm{wt} \%)$ & 6.6981 & 6.6002 & 7.1880 & -0.4899 & -0.5878 & $-6.8 \%$ & $-8.2 \%$ \\
\hline 18 & SB5VS-18 & $\mathrm{K} 2 \mathrm{O}(\mathrm{wt} \%)$ & 0.0313 & 0.0317 & 0.0323 & -0.0009 & -0.0005 & $-2.9 \%$ & $-1.7 \%$ \\
\hline 18 & SB5VS-18 & $\mathrm{La} 2 \mathrm{O} 3(\mathrm{wt} \%)$ & 0.0013 & 0.0013 & 0.0038 & -0.0025 & -0.0025 & $-65.6 \%$ & $-65.6 \%$ \\
\hline 18 & SB5VS-18 & $\mathrm{Li} 2 \mathrm{O}(\mathrm{wt} \%)$ & 5.6621 & 6.2183 & 6.0000 & -0.3379 & 0.2183 & $-5.6 \%$ & $3.6 \%$ \\
\hline 18 & SB5VS-18 & $\mathrm{MgO}(\mathrm{wt} \%)$ & 0.3304 & 0.3347 & 0.3860 & -0.0556 & -0.0513 & $-14.4 \%$ & $-13.3 \%$ \\
\hline 18 & SB5VS-18 & $\mathrm{MnO}\left(\mathrm{wt}^{2} \%\right)$ & 1.4946 & 1.4460 & 1.4858 & 0.0088 & -0.0398 & $0.6 \%$ & $-2.7 \%$ \\
\hline 18 & SB5VS-18 & $\mathrm{Na} 2 \mathrm{O}(\mathrm{wt} \%)$ & 11.2727 & 11.0315 & 11.8990 & -0.6263 & -0.8675 & $-5.3 \%$ & $-7.3 \%$ \\
\hline 18 & SB5VS-18 & $\mathrm{NiO}(\mathrm{wt} \%)$ & 0.7304 & 0.7119 & 0.7670 & -0.0366 & -0.0551 & $-4.8 \%$ & $-7.2 \%$ \\
\hline 18 & SB5VS-18 & $\mathrm{P} 2 \mathrm{O} 5(\mathrm{wt} \%)$ & 0.0206 & 0.0206 & 0.1063 & -0.0857 & -0.0857 & $-80.6 \%$ & $-80.6 \%$ \\
\hline 18 & SB5VS-18 & $\mathrm{PbO}(\mathrm{wt} \%)$ & 0.0110 & 0.0110 & 0.0045 & 0.0065 & 0.0065 & $145.4 \%$ & $145.4 \%$ \\
\hline 18 & SB5VS-18 & $\mathrm{SiO} 2(\mathrm{wt} \%)$ & 57.2263 & 58.2410 & 57.5370 & -0.3107 & 0.7040 & $-0.5 \%$ & $1.2 \%$ \\
\hline 18 & SB5VS-18 & SO4 (wt $\%)$ & 0.2172 & 0.2172 & 0.1831 & 0.0341 & 0.0341 & $18.6 \%$ & $18.6 \%$ \\
\hline 18 & SB5VS-18 & $\mathrm{TiO} 2(\mathrm{wt} \%)$ & 0.3386 & 0.3164 & 0.3268 & 0.0119 & -0.0103 & $3.6 \%$ & $-3.2 \%$ \\
\hline 18 & SB5VS-18 & U3O8 (wt\%) & 1.8219 & 1.9196 & 2.0180 & -0.1961 & $\begin{array}{l}-0.0984 \\
\end{array}$ & $-9.7 \%$ & $-4.9 \%$ \\
\hline 18 & SB5VS-18 & $\mathrm{ZnO}(\mathrm{wt} \%)$ & 0.0115 & 0.0115 & 0.0035 & 0.0080 & 0.0080 & $229.0 \%$ & $229.0 \%$ \\
\hline 18 & SB5VS-18 & $\mathrm{ZrO} 2(\mathrm{wt} \%)$ & 0.0311 & 0.0311 & 0.0375 & -0.0064 & -0.0064 & $-17.2 \%$ & $-17.2 \%$ \\
\hline 18 & SB5VS-18 & Sum & 97.6482 & 99.1757 & 99.9539 & -2.3057 & -0.7782 & $-2.3 \%$ & $-0.8 \%$ \\
\hline
\end{tabular}


Table 5 cont. Summary of the Target, Average Measured/Bias-Corrected Chemical Compositions

\begin{tabular}{|c|c|c|c|c|c|c|c|c|c|}
\hline & & & & Measured & & & & & \\
\hline & Glass & & Measured & Bias-Corrected & Targeted & Diff of & Diff of & $\%$ Diff of & $\%$ Diff of \\
\hline Glass \# & ID & Oxide & $(w t \%)$ & $(w t \%)$ & $(w t \%)$ & Measured & Meas BC & Measured & Meas BC \\
\hline 19 & SB5VS-19 & $\mathrm{Al} 2 \mathrm{O} 3(\mathrm{wt} \%)$ & 5.9378 & 6.0180 & 5.9156 & 0.0222 & 0.1025 & $0.4 \%$ & $1.7 \%$ \\
\hline 19 & SB5VS-19 & $\mathrm{B} 2 \mathrm{O} 3(\mathrm{wt} \%)$ & 5.5946 & 5.8128 & 5.7600 & -0.1654 & 0.0528 & $-2.9 \%$ & $0.9 \%$ \\
\hline 19 & SB5VS-19 & $\mathrm{BaO}(\mathrm{wt} \%)$ & 0.0396 & 0.0384 & 0.0389 & 0.0007 & -0.0005 & $1.8 \%$ & $-1.4 \%$ \\
\hline 19 & SB5VS-19 & $\mathrm{CaO}(\mathrm{wt} \%)$ & 0.6545 & 0.6582 & 0.6695 & -0.0150 & -0.0113 & $-2.2 \%$ & $-1.7 \%$ \\
\hline 19 & SB5VS-19 & $\mathrm{CdO}(\mathrm{wt} \%)$ & 0.0228 & 0.0228 & 0.0260 & -0.0032 & -0.0032 & $-12.3 \%$ & $-12.3 \%$ \\
\hline 19 & SB5VS-19 & $\mathrm{Ce} 2 \mathrm{O} 3(\mathrm{wt} \%)$ & 0.0237 & 0.0237 & 0.0067 & 0.0170 & 0.0170 & $253.0 \%$ & $253.0 \%$ \\
\hline 19 & SB5VS-19 & $\mathrm{Cr} 2 \mathrm{O} 3(\mathrm{wt} \%)$ & 0.0281 & 0.0276 & 0.0286 & -0.0004 & -0.0009 & $-1.5 \%$ & $-3.2 \%$ \\
\hline 19 & SB5VS-19 & $\mathrm{CuO}(\mathrm{wt} \%)$ & 0.0141 & 0.0140 & 0.0073 & 0.0068 & 0.0068 & $93.4 \%$ & $92.8 \%$ \\
\hline 19 & SB5VS-19 & $\mathrm{Fe} 2 \mathrm{O} 3(\mathrm{wt} \%)$ & 7.5846 & 7.6077 & 8.0506 & -0.4660 & -0.4429 & $-5.8 \%$ & $-5.5 \%$ \\
\hline 19 & SB5VS-19 & $\mathrm{K} 2 \mathrm{O}(\mathrm{wt} \%)$ & 0.0373 & 0.0368 & 0.0361 & 0.0012 & 0.0007 & $3.4 \%$ & $1.9 \%$ \\
\hline 19 & SB5VS-19 & $\mathrm{La} 2 \mathrm{O} 3(\mathrm{wt} \%)$ & 0.0010 & 0.0010 & 0.0042 & -0.0032 & -0.0032 & $-76.3 \%$ & $-76.3 \%$ \\
\hline 19 & SB5VS-19 & $\mathrm{Li} 2 \mathrm{O}(\mathrm{wt} \%)$ & 5.5545 & 6.0996 & 5.7600 & -0.2055 & 0.3396 & $-3.6 \%$ & $5.9 \%$ \\
\hline 19 & SB5VS-19 & $\mathrm{MgO}\left(\mathrm{wt}^{2} \%\right)$ & 0.4428 & 0.4267 & 0.4323 & 0.0104 & -0.0056 & $2.4 \%$ & $-1.3 \%$ \\
\hline 19 & SB5VS-19 & $\mathrm{MnO}(\mathrm{wt} \%)$ & 1.6592 & 1.6573 & 1.6640 & -0.0048 & -0.0068 & $-0.3 \%$ & $-0.4 \%$ \\
\hline 19 & SB5VS-19 & $\mathrm{Na} 2 \mathrm{O}(\mathrm{wt} \%)$ & 12.3814 & 12.2355 & 12.3669 & 0.0145 & -0.1314 & $0.1 \%$ & $-1.1 \%$ \\
\hline 19 & SB5VS-19 & $\mathrm{NiO}(\mathrm{wt} \%)$ & 0.8325 & 0.8180 & 0.8590 & -0.0265 & -0.0410 & $-3.1 \%$ & $-4.8 \%$ \\
\hline 19 & SB5VS-19 & P2O5 (wt\%) & 0.0550 & 0.0550 & 0.1191 & -0.0641 & -0.0641 & $-53.8 \%$ & $-53.8 \%$ \\
\hline 19 & SB5VS-19 & $\mathrm{PbO}(\mathrm{wt} \%)$ & 0.0108 & 0.0108 & 0.0050 & 0.0057 & 0.0057 & $113.7 \%$ & $113.7 \%$ \\
\hline 19 & SB5VS-19 & $\mathrm{SiO} 2(\mathrm{wt} \%)$ & 54.7661 & 55.7297 & 55.3220 & -0.5559 & 0.4077 & $-1.0 \%$ & $0.7 \%$ \\
\hline 19 & SB5VS-19 & $\mathrm{SO} 4(\mathrm{wt} \%)$ & 0.2314 & 0.2314 & 0.2051 & 0.0264 & 0.0264 & $12.9 \%$ & $12.9 \%$ \\
\hline 19 & SB5VS-19 & $\mathrm{TiO} 2(\mathrm{wt} \%)$ & 0.3690 & 0.3621 & 0.3660 & 0.0031 & -0.0039 & $0.8 \%$ & $-1.1 \%$ \\
\hline 19 & SB5VS-19 & U3O8 (wt\%) & 2.1461 & 2.2004 & 2.2602 & -0.1140 & -0.0597 & $-5.0 \%$ & $-2.6 \%$ \\
\hline 19 & SB5VS-19 & $\mathrm{ZnO}(\mathrm{wt} \%)$ & 0.0103 & 0.0103 & 0.0039 & 0.0063 & 0.0063 & $162.0 \%$ & $162.0 \%$ \\
\hline 19 & SB5VS-19 & $\mathrm{ZrO} 2(\mathrm{wt} \%)$ & 0.0446 & 0.0446 & 0.0420 & 0.0026 & 0.0026 & $6.1 \%$ & $6.1 \%$ \\
\hline 19 & SB5VS-19 & Sum & 98.4418 & 100.1425 & 99.9489 & -1.5072 & 0.1935 & $-1.5 \%$ & $0.2 \%$ \\
\hline 20 & SB5VS-20 & $\mathrm{Al} 2 \mathrm{O} 3(\mathrm{wt} \%)$ & 6.4196 & 6.4852 & 6.5494 & -0.1298 & -0.0642 & $-2.0 \%$ & $-1.0 \%$ \\
\hline 20 & SB5VS-20 & $\mathrm{B} 2 \mathrm{O} 3(\mathrm{wt} \%)$ & 5.5946 & 5.5232 & 5.5200 & 0.0746 & 0.0032 & $1.4 \%$ & $0.1 \%$ \\
\hline 20 & SB5VS-20 & $\mathrm{BaO}(\mathrm{wt} \%)$ & 0.0486 & 0.0463 & 0.0431 & 0.0055 & 0.0032 & $12.7 \%$ & $7.5 \%$ \\
\hline 20 & SB5VS-20 & $\mathrm{CaO}(\mathrm{wt} \%)$ & 0.7108 & 0.7091 & 0.7412 & -0.0304 & -0.0321 & $-4.1 \%$ & $-4.3 \%$ \\
\hline 20 & SB5VS-20 & $\mathrm{CdO}(\mathrm{wt} \%)$ & 0.0263 & 0.0263 & 0.0288 & -0.0026 & -0.0026 & $-8.9 \%$ & $-8.9 \%$ \\
\hline 20 & SB5VS-20 & $\mathrm{Ce} 2 \mathrm{O} 3(\mathrm{wt} \%)$ & 0.0258 & 0.0258 & 0.0074 & 0.0183 & 0.0183 & $246.4 \%$ & $246.4 \%$ \\
\hline 20 & SB5VS-20 & $\mathrm{Cr} 2 \mathrm{O} 3(\mathrm{wt} \%)$ & 0.0307 & 0.0290 & 0.0316 & -0.0009 & -0.0026 & $-2.9 \%$ & $-8.3 \%$ \\
\hline 20 & SB5VS-20 & $\mathrm{CuO}(\mathrm{wt} \%)$ & 0.0169 & 0.0164 & 0.0081 & 0.0088 & 0.0083 & $109.7 \%$ & $103.2 \%$ \\
\hline 20 & SB5VS-20 & $\mathrm{Fe} 2 \mathrm{O} 3(\mathrm{wt} \%)$ & 8.3888 & 8.3256 & 8.9131 & -0.5244 & -0.5875 & $-5.9 \%$ & $-6.6 \%$ \\
\hline 20 & SB5VS-20 & $\mathrm{K} 2 \mathrm{O}(\mathrm{wt} \%)$ & 0.0401 & 0.0399 & 0.0400 & 0.0001 & -0.0001 & $0.2 \%$ & $-0.3 \%$ \\
\hline 20 & SB5VS-20 & $\mathrm{La} 2 \mathrm{O} 3(\mathrm{wt} \%)$ & 0.0001 & 0.0001 & 0.0047 & -0.0045 & -0.0045 & $-97.5 \%$ & $-97.5 \%$ \\
\hline 20 & SB5VS-20 & $\mathrm{Li} 2 \mathrm{O}(\mathrm{wt} \%)$ & 5.1777 & 5.6459 & 5.5200 & -0.3423 & 0.1259 & $-6.2 \%$ & $2.3 \%$ \\
\hline 20 & SB5VS-20 & $\mathrm{MgO}(\mathrm{wt} \%)$ & 0.4730 & 0.4617 & 0.4786 & -0.0056 & -0.0169 & $-1.2 \%$ & $-3.5 \%$ \\
\hline 20 & SB5VS-20 & $\mathrm{MnO}\left(\mathrm{wt}^{2} \%\right)$ & 1.8174 & 1.7735 & 1.8423 & -0.0250 & -0.0688 & $-1.4 \%$ & $-3.7 \%$ \\
\hline 20 & SB5VS-20 & $\mathrm{Na} 2 \mathrm{O}(\mathrm{wt} \%)$ & 12.4892 & 12.1740 & 12.8348 & -0.3455 & -0.6607 & $-2.7 \%$ & $-5.1 \%$ \\
\hline 20 & SB5VS-20 & $\mathrm{NiO}(\mathrm{wt} \%)$ & 0.9089 & 0.8696 & 0.9511 & -0.0422 & -0.0815 & $-4.4 \%$ & $-8.6 \%$ \\
\hline 20 & SB5VS-20 & $\mathrm{P} 2 \mathrm{O} 5(\mathrm{wt} \%)$ & 0.0395 & 0.0395 & 0.1318 & -0.0923 & -0.0923 & $-70.0 \%$ & $-70.0 \%$ \\
\hline 20 & SB5VS-20 & $\mathrm{PbO}(\mathrm{wt} \%)$ & 0.0113 & 0.0113 & 0.0056 & 0.0057 & 0.0057 & $102.7 \%$ & $102.7 \%$ \\
\hline 20 & SB5VS-20 & $\mathrm{SiO} 2(\mathrm{wt} \%)$ & 49.9527 & 52.2083 & 53.1060 & -3.1533 & -0.8977 & $-5.9 \%$ & $-1.7 \%$ \\
\hline 20 & SB5VS-20 & SO4 (wt\%) & 0.2517 & 0.2517 & 0.2270 & 0.0246 & 0.0246 & $10.8 \%$ & $10.8 \%$ \\
\hline 20 & SB5VS-20 & $\mathrm{TiO} 2(\mathrm{wt} \%)$ & 0.4007 & 0.3926 & 0.4052 & -0.0044 & -0.0126 & $-1.1 \%$ & $-3.1 \%$ \\
\hline 20 & SB5VS-20 & U3O8 (wt\%) & 2.3496 & 2.4131 & 2.5023 & -0.1528 & $\begin{array}{l}-0.0893 \\
\end{array}$ & $-6.1 \%$ & $-3.6 \%$ \\
\hline 20 & SB5VS-20 & $\mathrm{ZnO}(\mathrm{wt} \%)$ & 0.0112 & 0.0112 & 0.0043 & 0.0069 & 0.0069 & $158.1 \%$ & $158.1 \%$ \\
\hline 20 & SB5VS-20 & $\mathrm{ZrO} 2(\mathrm{wt} \%)$ & 0.0493 & 0.0493 & 0.0465 & 0.0028 & 0.0028 & $6.0 \%$ & $6.0 \%$ \\
\hline 20 & SB5VS-20 & Sum & 95.2343 & 97.5285 & 99.9430 & -4.7087 & -2.4145 & $-4.7 \%$ & $-2.4 \%$ \\
\hline
\end{tabular}


Table 5 cont. Summary of the Target, Average Measured/Bias-Corrected Chemical Compositions

\begin{tabular}{|c|c|c|c|c|c|c|c|c|c|}
\hline & & & & Measured & & & & & \\
\hline & Glass & & Measured & Bias-Corrected & Targeted & Diff of & Diff of & $\%$ Diff of & $\%$ Diff of \\
\hline Glass \# & ID & Oxide & $(w t \%)$ & $(w t \%)$ & $(w t \%)$ & Measured & Meas BC & Measured & Meas BC \\
\hline 21 & SB5VS-21 & $\mathrm{A} 12 \mathrm{O} 3(\mathrm{wt} \%)$ & 7.3596 & 7.4348 & 7.8170 & -0.4574 & -0.3822 & $-5.9 \%$ & $-4.9 \%$ \\
\hline 21 & SB5VS-21 & $\mathrm{B} 2 \mathrm{O} 3(\mathrm{wt} \%)$ & 5.3370 & 5.2689 & 5.0400 & 0.2970 & 0.2289 & $5.9 \%$ & $4.5 \%$ \\
\hline 21 & SB5VS-21 & $\mathrm{BaO}(\mathrm{wt} \%)$ & 0.0522 & 0.0498 & 0.0514 & 0.0008 & -0.0017 & $1.5 \%$ & $-3.2 \%$ \\
\hline 21 & SB5VS-21 & $\mathrm{CaO}(\mathrm{wt} \%)$ & 0.8255 & 0.8236 & 0.8847 & -0.0591 & -0.0611 & $-6.7 \%$ & $-6.9 \%$ \\
\hline 21 & SB5VS-21 & $\mathrm{CdO}\left(\mathrm{wt}^{\circ} \%\right)$ & 0.0320 & 0.0320 & 0.0344 & -0.0024 & -0.0024 & $-7.0 \%$ & $-7.0 \%$ \\
\hline 21 & SB5VS-21 & $\mathrm{Ce} 2 \mathrm{O} 3(\mathrm{wt} \%)$ & 0.0284 & 0.0284 & 0.0089 & 0.0195 & 0.0195 & $219.9 \%$ & $219.9 \%$ \\
\hline 21 & SB5VS-21 & $\mathrm{Cr} 2 \mathrm{O} 3(\mathrm{wt} \%)$ & 0.0340 & 0.0321 & 0.0377 & -0.0038 & -0.0056 & $-10.0 \%$ & $-15.0 \%$ \\
\hline 21 & SB5VS-21 & $\mathrm{CuO}(\mathrm{wt} \%)$ & 0.0166 & 0.0161 & 0.0096 & 0.0070 & 0.0065 & $72.4 \%$ & $67.1 \%$ \\
\hline 21 & SB5VS-21 & $\mathrm{Fe} 2 \mathrm{O} 3(\mathrm{wt} \%)$ & 9.7506 & 9.6772 & 10.6382 & -0.8877 & -0.9611 & $-8.3 \%$ & $-9.0 \%$ \\
\hline 21 & SB5VS-21 & $\mathrm{K} 2 \mathrm{O}(\mathrm{wt} \%)$ & 0.0461 & 0.0459 & 0.0477 & -0.0017 & -0.0019 & $-3.5 \%$ & $-3.9 \%$ \\
\hline 21 & SB5VS-21 & $\mathrm{La} 2 \mathrm{O} 3(\mathrm{wt} \%)$ & 0.0001 & 0.0001 & 0.0056 & -0.0054 & -0.0054 & $-97.9 \%$ & $-97.9 \%$ \\
\hline 21 & SB5VS-21 & $\mathrm{Li} 2 \mathrm{O}(\mathrm{wt} \%)$ & 4.6556 & 5.0761 & 5.0400 & -0.3844 & 0.0361 & $-7.6 \%$ & $0.7 \%$ \\
\hline 21 & SB5VS-21 & $\mathrm{MgO}(\mathrm{wt} \%)$ & 0.5414 & 0.5285 & 0.5713 & -0.0298 & -0.0428 & $-5.2 \%$ & $-7.5 \%$ \\
\hline 21 & SB5VS-21 & $\mathrm{MnO}(\mathrm{wt} \%)$ & 2.0627 & 2.0133 & 2.1989 & -0.1362 & -0.1856 & $-6.2 \%$ & $-8.4 \%$ \\
\hline 21 & SB5VS-21 & $\mathrm{Na} 2 \mathrm{O}(\mathrm{wt} \%)$ & 13.2205 & 12.8869 & 13.7705 & -0.5500 & -0.8837 & $-4.0 \%$ & $-6.4 \%$ \\
\hline 21 & SB5VS-21 & $\mathrm{NiO}(\mathrm{wt} \%)$ & 1.0326 & 0.9880 & 1.1352 & -0.1025 & -0.1472 & $-9.0 \%$ & $-13.0 \%$ \\
\hline 21 & SB5VS-21 & $\mathrm{P} 2 \mathrm{O} 5(\mathrm{wt} \%)$ & 0.0613 & 0.0613 & 0.1573 & -0.0960 & -0.0960 & $-61.0 \%$ & $-61.0 \%$ \\
\hline 21 & SB5VS-21 & $\mathrm{PbO}(\mathrm{wt} \%)$ & 0.0108 & 0.0108 & 0.0067 & 0.0041 & 0.0041 & $61.7 \%$ & $61.7 \%$ \\
\hline 21 & SB5VS-21 & $\mathrm{SiO} 2(\mathrm{wt} \%)$ & 47.5459 & 49.6819 & 48.6750 & -1.1291 & 1.0069 & $-2.3 \%$ & $2.1 \%$ \\
\hline 21 & SB5VS-21 & SO4 (wt $\%)$ & 0.3176 & 0.3176 & 0.2710 & 0.0466 & 0.0466 & $17.2 \%$ & $17.2 \%$ \\
\hline 21 & SB5VS-21 & $\mathrm{TiO} 2(\mathrm{wt} \%)$ & 0.4720 & 0.4624 & 0.4836 & -0.0115 & -0.0212 & $-2.4 \%$ & $-4.4 \%$ \\
\hline 21 & SB5VS-21 & U3O8 (wt\%) & 2.7004 & 2.7734 & 2.9866 & -0.2863 & -0.2132 & $-9.6 \%$ & $-7.1 \%$ \\
\hline 21 & SB5VS-21 & $\mathrm{ZnO}(\mathrm{wt} \%)$ & 0.0124 & 0.0124 & 0.0052 & 0.0073 & 0.0073 & $140.3 \%$ & $140.3 \%$ \\
\hline 21 & SB5VS-21 & $\mathrm{ZrO} 2\left(\mathrm{wt}^{\circ} \%\right)$ & 0.0527 & 0.0527 & 0.0555 & -0.0028 & -0.0028 & $-5.1 \%$ & $-5.1 \%$ \\
\hline 21 & SB5VS-21 & Sum & 96.1680 & 98.2741 & 99.9320 & -3.7640 & -1.6580 & $-3.8 \%$ & $-1.7 \%$ \\
\hline 100 & Batch 1 & $\mathrm{~A} 12 \mathrm{O} 3(\mathrm{wt} \%)$ & 4.8245 & 4.8770 & 4.8770 & -0.0525 & 0.0000 & $-1.1 \%$ & $0.0 \%$ \\
\hline 100 & Batch 1 & $\mathrm{~B} 2 \mathrm{O} 3(\mathrm{wt} \%)$ & 7.6043 & 7.7770 & 7.7770 & -0.1727 & 0.0000 & $-2.2 \%$ & $0.0 \%$ \\
\hline 100 & Batch 1 & $\mathrm{BaO}(\mathrm{wt} \%)$ & 0.1577 & 0.1510 & 0.1510 & 0.0067 & 0.0000 & $4.4 \%$ & $0.0 \%$ \\
\hline 100 & Batch 1 & $\mathrm{CaO}(\mathrm{wt} \%)$ & 1.2137 & 1.2200 & 1.2200 & -0.0063 & 0.0000 & $-0.5 \%$ & $0.0 \%$ \\
\hline 100 & Batch 1 & $\mathrm{CdO}(\mathrm{wt} \%)$ & 0.0010 & 0.0010 & 0.0000 & 0.0010 & 0.0010 & & \\
\hline 100 & Batch 1 & $\mathrm{Ce} 2 \mathrm{O} 3(\mathrm{wt} \%)$ & 0.0023 & 0.0023 & 0.0000 & 0.0023 & 0.0023 & & \\
\hline 100 & Batch 1 & $\mathrm{Cr} 2 \mathrm{O} 3(\mathrm{wt} \%)$ & 0.1111 & 0.1070 & 0.1070 & 0.0041 & 0.0000 & $3.8 \%$ & $0.0 \%$ \\
\hline 100 & Batch 1 & $\mathrm{CuO}\left(\mathrm{wt}^{\circ} \%\right)$ & 0.4047 & 0.3990 & 0.3990 & 0.0057 & 0.0000 & $1.4 \%$ & $0.0 \%$ \\
\hline 100 & Batch 1 & $\mathrm{Fe} 2 \mathrm{O} 3(\mathrm{wt} \%)$ & 12.9221 & 12.8390 & 12.8390 & 0.0831 & 0.0000 & $0.6 \%$ & $0.0 \%$ \\
\hline 100 & Batch 1 & $\mathrm{~K} 2 \mathrm{O}(\mathrm{wt} \%)$ & 3.3354 & 3.3270 & 3.3270 & 0.0084 & 0.0000 & $0.3 \%$ & $0.0 \%$ \\
\hline 100 & Batch 1 & $\mathrm{La} 2 \mathrm{O} 3(\mathrm{wt} \%)$ & 0.0005 & 0.0005 & 0.0000 & 0.0005 & 0.0005 & & \\
\hline 100 & Batch 1 & $\mathrm{Li} 2 \mathrm{O}(\mathrm{wt} \%)$ & 4.0152 & 4.4290 & 4.4290 & -0.4138 & 0.0000 & $-9.3 \%$ & $0.0 \%$ \\
\hline 100 & Batch 1 & $\mathrm{MgO}(\mathrm{wt} \%)$ & 1.4422 & 1.4190 & 1.4190 & 0.0232 & 0.0000 & $1.6 \%$ & $0.0 \%$ \\
\hline 100 & Batch 1 & $\mathrm{MnO}(\mathrm{wt} \%)$ & 1.7603 & 1.7260 & 1.7260 & 0.0343 & 0.0000 & $2.0 \%$ & $0.0 \%$ \\
\hline 100 & Batch 1 & $\mathrm{Na} 2 \mathrm{O}(\mathrm{wt} \%)$ & 9.1821 & 9.0030 & 9.0030 & 0.1791 & 0.0000 & $2.0 \%$ & $0.0 \%$ \\
\hline 100 & Batch 1 & $\mathrm{NiO}(\mathrm{wt} \%)$ & 0.7733 & 0.7510 & 0.7510 & 0.0223 & 0.0000 & $3.0 \%$ & $0.0 \%$ \\
\hline 100 & Batch 1 & P2O5 (wt $\%)$ & 0.0206 & 0.0206 & 0.0000 & 0.0206 & 0.0206 & & \\
\hline 100 & Batch 1 & $\mathrm{PbO}(\mathrm{wt} \%)$ & 0.0066 & 0.0066 & 0.0000 & 0.0066 & 0.0066 & & \\
\hline 100 & Batch 1 & $\mathrm{SiO} 2\left(w t^{\circ} \%\right)$ & 49.3109 & 50.2200 & 50.2200 & -0.9091 & 0.0000 & $-1.8 \%$ & $0.0 \%$ \\
\hline 100 & Batch 1 & SO4 (wt \%) & 0.1330 & 0.1330 & 0.0000 & 0.1330 & 0.1330 & & \\
\hline 100 & Batch 1 & $\mathrm{TiO} 2(\mathrm{wt} \%)$ & 0.7019 & 0.6770 & 0.6770 & 0.0249 & 0.0000 & $3.7 \%$ & $0.0 \%$ \\
\hline 100 & Batch 1 & U3O8 (wt\%) & 0.0884 & 0.0916 & 0.0000 & 0.0884 & 0.0916 & & \\
\hline 100 & Batch 1 & $\mathrm{ZnO}(\mathrm{wt} \%)$ & 0.0108 & 0.0108 & 0.0000 & 0.0108 & 0.0108 & & \\
\hline 100 & Batch 1 & $\mathrm{ZrO} 2(\mathrm{wt} \%)$ & 0.0984 & 0.0984 & 0.0980 & 0.0004 & 0.0004 & $0.4 \%$ & $0.4 \%$ \\
\hline 100 & Batch 1 & Sum & 98.1211 & 99.2869 & 99.0200 & -0.8989 & 0.2669 & $-0.9 \%$ & $0.3 \%$ \\
\hline
\end{tabular}


SRNS-STI-2008-00065

Revision 0

Table 5 cont. Summary of the Target, Average Measured/Bias-Corrected Chemical Compositions

\begin{tabular}{|c|c|c|c|c|c|c|c|c|c|}
\hline & & & & Measured & & & & & \\
\hline & Glass & & Measured & Bias-Corrected & Targeted & Diff of & Diff of & $\%$ Diff of & $\%$ Diff of \\
\hline Glass \# & ID & Oxide & $(w t \%)$ & $(w t \%)$ & $(\mathrm{wt} \%)$ & Measured & Meas BC & Measured & Meas BC \\
\hline 200 & Ustd & $\mathrm{A} 12 \mathrm{O} 3(\mathrm{wt} \%)$ & 3.8661 & 3.9082 & 4.1000 & -0.2339 & -0.1918 & $-5.7 \%$ & $-4.7 \%$ \\
\hline 200 & Ustd & $\mathrm{B} 2 \mathrm{O} 3(\mathrm{wt} \%)$ & 9.4200 & 9.6356 & 9.2090 & 0.2110 & 0.4266 & $2.3 \%$ & $4.6 \%$ \\
\hline 200 & Ustd & $\mathrm{BaO}(\mathrm{wt} \%)$ & 0.0014 & 0.0014 & 0.0000 & 0.0014 & 0.0014 & & \\
\hline 200 & Ustd & $\mathrm{CaO}(\mathrm{wt} \%)$ & 1.2681 & 1.2746 & 1.3010 & -0.0329 & -0.0264 & $-2.5 \%$ & $-2.0 \%$ \\
\hline 200 & Ustd & $\mathrm{CdO}(\mathrm{wt} \%)$ & 0.0003 & 0.0003 & 0.0000 & 0.0003 & 0.0003 & & \\
\hline 200 & Ustd & $\mathrm{Ce} 2 \mathrm{O} 3(\mathrm{wt} \%)$ & 0.0171 & 0.0171 & 0.0000 & 0.0171 & 0.0171 & & \\
\hline 200 & Ustd & $\mathrm{Cr} 2 \mathrm{O} 3(\mathrm{wt} \%)$ & 0.2550 & 0.2457 & 0.0000 & 0.2550 & 0.2457 & & \\
\hline 200 & Ustd & $\mathrm{CuO}(\mathrm{wt} \%)$ & 0.0114 & 0.0112 & 0.0000 & 0.0114 & 0.0112 & & \\
\hline 200 & Ustd & $\mathrm{Fe} 2 \mathrm{O} 3(\mathrm{wt} \%)$ & 13.1096 & 13.0261 & 13.1960 & -0.0864 & -0.1699 & $-0.7 \%$ & $-1.3 \%$ \\
\hline 200 & Ustd & $\mathrm{K} 2 \mathrm{O}(\mathrm{wt} \%)$ & 2.9098 & 2.9022 & 2.9990 & -0.0892 & -0.0968 & $-3.0 \%$ & $-3.2 \%$ \\
\hline 200 & Ustd & $\mathrm{La} 2 \mathrm{O} 3(\mathrm{wt} \%)$ & 0.0001 & 0.0001 & 0.0000 & 0.0001 & 0.0001 & & \\
\hline 200 & Ustd & $\mathrm{Li} 2 \mathrm{O}(\mathrm{wt} \%)$ & 2.8502 & 3.1437 & 3.0570 & -0.2068 & 0.0867 & $-6.8 \%$ & $2.8 \%$ \\
\hline 200 & Ustd & $\mathrm{MgO}(\mathrm{wt} \%)$ & 1.1955 & 1.1761 & 1.2100 & -0.0145 & -0.0339 & $-1.2 \%$ & $-2.8 \%$ \\
\hline 200 & Ustd & $\mathrm{MnO}\left(\mathrm{wt}^{\circ} \%\right)$ & 2.7596 & 2.7064 & 2.8920 & -0.1324 & -0.1856 & $-4.6 \%$ & $-6.4 \%$ \\
\hline 200 & Ustd & $\mathrm{Na} 2 \mathrm{O}(\mathrm{wt} \%)$ & 11.7119 & 11.4841 & 11.7950 & -0.0831 & -0.3109 & $-0.7 \%$ & $-2.6 \%$ \\
\hline 200 & Ustd & $\mathrm{NiO}(\mathrm{wt} \%)$ & 1.0602 & 1.0298 & 1.1200 & -0.0598 & -0.0902 & $-5.3 \%$ & $-8.1 \%$ \\
\hline 200 & Ustd & $\mathrm{P} 2 \mathrm{O} 5(\mathrm{wt} \%)$ & 0.0206 & 0.0206 & 0.0000 & 0.0206 & 0.0206 & & \\
\hline 200 & Ustd & $\mathrm{PbO}(\mathrm{wt} \%)$ & 0.0032 & 0.0032 & 0.0000 & 0.0032 & 0.0032 & & \\
\hline 200 & Ustd & $\mathrm{SiO} 2(w t \%)$ & 47.0052 & 47.8674 & 45.3530 & 1.6522 & 2.5144 & $3.6 \%$ & $5.5 \%$ \\
\hline 200 & Ustd & SO4 (wt $\%)$ & 0.1070 & 0.1070 & 0.0000 & 0.1070 & 0.1070 & & \\
\hline 200 & Ustd & $\mathrm{TiO} 2\left(\mathrm{wt}^{\mathrm{T}} \%\right)$ & 0.9917 & 0.9567 & 1.0490 & -0.0573 & -0.0923 & $-5.5 \%$ & $-8.8 \%$ \\
\hline 200 & Ustd & U3O8 (wt\%) & 2.3243 & 2.4060 & 2.4060 & -0.0817 & 0.0000 & $-3.4 \%$ & $0.0 \%$ \\
\hline 200 & Ustd & $\mathrm{ZnO}(\mathrm{wt} \%)$ & 0.0097 & 0.0097 & 0.0000 & 0.0097 & 0.0097 & & \\
\hline 200 & Ustd & $\mathrm{ZrO} 2(\mathrm{wt} \%)$ & 0.0032 & 0.0032 & 0.0000 & 0.0032 & 0.0032 & & \\
\hline 200 & Ustd & Sum & 100.9013 & 101.9366 & 99.6870 & 1.2143 & 2.2496 & $1.2 \%$ & $2.3 \%$ \\
\hline
\end{tabular}


Table 6. Normalized PCT Data

\begin{tabular}{|c|c|c|c|c|c|c|c|c|c|c|c|c|c|}
\hline Glass ID & WL $(\%)$ & Frit & Sludge Case & $\begin{array}{c}\text { Heat } \\
\text { Treatment }\end{array}$ & Comp View & $\begin{array}{c}\log \mathrm{NL} \\
{[\mathrm{B}(\mathrm{g} / \mathrm{L})]}\end{array}$ & $\begin{array}{c}\log \mathrm{NL} \\
{[\mathrm{Li}(\mathrm{g} / \mathrm{L})]}\end{array}$ & $\begin{array}{c}\log \mathrm{NL} \\
{[\mathrm{Na}(\mathrm{g} / \mathrm{L})]}\end{array}$ & $\begin{array}{c}\log \mathrm{NL} \\
{[\mathrm{Si}(\mathrm{g} / \mathrm{L})]}\end{array}$ & $\begin{array}{c}\mathrm{NL} \\
\mathrm{B}(\mathrm{g} / \mathrm{L})\end{array}$ & $\begin{array}{c}\mathrm{NL} \\
\mathrm{Li}(\mathrm{g} / \mathrm{L})\end{array}$ & $\begin{array}{c}\mathrm{NL} \\
\mathrm{Na}(\mathrm{g} / \mathrm{L})\end{array}$ & $\begin{array}{c}\mathrm{NL} \\
\mathrm{Si}(\mathrm{g} / \mathrm{L})\end{array}$ \\
\hline ARM & 0 & ref & ref & ref & reference & -0.2748 & -0.1767 & -0.2695 & -0.5202 & 0.531 & 0.666 & 0.538 & 0.302 \\
\hline EA & 0 & ref & ref & ref & reference & 1.2382 & 1.0154 & 1.1201 & 0.6070 & 17.307 & 10.360 & 13.185 & 4.046 \\
\hline ARM & 0 & ref & ref & ref & reference & -0.3218 & -0.2304 & -0.2885 & -0.5493 & 0.477 & 0.588 & 0.515 & 0.282 \\
\hline EA & 0 & ref & ref & ref & reference & 1.2328 & 0.9993 & 1.1233 & 0.6075 & 17.094 & 9.984 & 13.283 & 4.051 \\
\hline SB5VS-01 & 34 & 418 & D-Optimal EV & $\mathrm{ccc}$ & targeted & -0.0803 & 0.0473 & -0.0309 & -0.1773 & 0.831 & 1.115 & 0.931 & 0.665 \\
\hline $\begin{array}{l}\text { SB5VS-01 } \\
\end{array}$ & 34 & 418 & D-Optimal EV & quenched & targeted & -0.1051 & 0.0125 & -0.0121 & -0.1808 & 0.785 & 1.029 & 0.973 & 0.660 \\
\hline SB5VS-01 & 34 & 418 & D-Optimal EV & $\mathrm{ccc}$ & Measured & -0.0914 & 0.0951 & -0.0332 & -0.1744 & 0.810 & 1.245 & 0.926 & 0.669 \\
\hline SB5VS-01 & 34 & 418 & D-Optimal EV & quenched & Measured & -0.1163 & 0.0602 & -0.0144 & -0.1778 & 0.765 & 1.149 & 0.967 & 0.664 \\
\hline SB5VS-01 & 34 & 418 & D-Optimal EV & $\mathrm{ccc}$ & Measured bc & -0.1101 & 0.0455 & -0.0281 & -0.1718 & 0.776 & 1.110 & 0.937 & 0.673 \\
\hline SB5VS-01 & 34 & 418 & D-Optimal EV & quenched & Measured bc & -0.1349 & 0.0106 & -0.0093 & -0.1752 & 0.733 & 1.025 & 0.979 & 0.668 \\
\hline SB5VS-02 & 34 & 418 & D-Optimal EV & $\mathrm{ccc}$ & targeted & -0.1200 & 0.0052 & -0.0416 & -0.1938 & 0.759 & 1.012 & 0.909 & 0.640 \\
\hline SB5VS-02 & 34 & 418 & D-Optimal EV & quenched & targeted & -0.1124 & -0.0095 & -0.0080 & -0.1916 & 0.772 & 0.978 & 0.982 & 0.643 \\
\hline SB5VS-02 & 34 & 418 & D-Optimal EV & $\mathrm{ccc}$ & Measured & -0.1351 & 0.0290 & -0.0204 & -0.1696 & 0.733 & 1.069 & 0.954 & 0.677 \\
\hline SB5VS-02 & 34 & 418 & D-Optimal EV & quenched & Measured & -0.1274 & 0.0142 & 0.0133 & -0.1674 & 0.746 & 1.033 & 1.031 & 0.680 \\
\hline SB5VS-02 & 34 & 418 & D-Optimal EV & $\mathrm{ccc}$ & Measured bc & -0.1295 & -0.0085 & -0.0093 & -0.1888 & 0.742 & 0.981 & 0.979 & 0.647 \\
\hline SB5VS-02 & 34 & 418 & D-Optimal EV & quenched & Measured bc & -0.1219 & -0.0232 & 0.0244 & -0.1866 & 0.755 & 0.948 & 1.058 & 0.651 \\
\hline SB5VS-03 & 34 & 418 & D-Optimal EV & $\mathrm{ccc}$ & targeted & -0.0767 & 0.0119 & -0.0711 & -0.2273 & 0.838 & 1.028 & 0.849 & 0.593 \\
\hline SB5VS-03 & 34 & 418 & D-Optimal EV & quenched & targeted & -0.0737 & 0.0034 & -0.0508 & -0.2173 & 0.844 & 1.008 & 0.890 & 0.606 \\
\hline SB5VS-03 & 34 & 418 & D-Optimal EV & $\mathrm{ccc}$ & Measured & -0.0366 & 0.0661 & -0.0668 & -0.2013 & 0.919 & 1.164 & 0.858 & 0.629 \\
\hline $\begin{array}{l}\text { SB5VS-03 } \\
\end{array}$ & 34 & 418 & D-Optimal EV & quenched & Measured & -0.0336 & 0.0576 & -0.0465 & -0.1914 & 0.926 & 1.142 & 0.899 & 0.644 \\
\hline SB5VS-03 & 34 & 418 & D-Optimal EV & $\mathrm{ccc}$ & Measured bc & -0.0553 & 0.0164 & -0.0616 & $\begin{array}{l}-0.1988 \\
\end{array}$ & 0.880 & 1.039 & 0.868 & 0.633 \\
\hline $\begin{array}{l}\text { SB5VS-03 } \\
\end{array}$ & 34 & 418 & D-Optimal EV & quenched & Measured bc & -0.0523 & 0.0079 & -0.0413 & -0.1888 & 0.886 & 1.018 & 0.909 & 0.647 \\
\hline SB5VS-04 & 34 & 418 & D-Optimal EV & $\mathrm{ccc}$ & targeted & -0.0083 & 0.1334 & 0.1034 & -0.1185 & 0.981 & 1.360 & 1.269 & 0.761 \\
\hline $\begin{array}{l}\text { SB5VS-04 } \\
\end{array}$ & 34 & 418 & D-Optimal EV & quenched & targeted & -0.0110 & 0.1068 & 0.1329 & -0.1092 & 0.975 & 1.279 & 1.358 & 0.778 \\
\hline SB5VS-04 & 34 & 418 & D-Optimal EV & ccc & Measured & -0.0227 & 0.1712 & 0.1226 & -0.0890 & 0.949 & 1.483 & 1.326 & 0.815 \\
\hline $\begin{array}{l}\text { SB5VS-04 } \\
\end{array}$ & 34 & 418 & D-Optimal EV & quenched & Measured & -0.0254 & 0.1445 & 0.1521 & -0.0796 & 0.943 & 1.395 & 1.419 & 0.832 \\
\hline SB5VS-04 & 34 & 418 & D-Optimal EV & $\mathrm{ccc}$ & Measured bc & -0.0171 & 0.1336 & 0.1337 & -0.1081 & 0.961 & 1.360 & 1.361 & 0.780 \\
\hline SB5VS-04 & 34 & 418 & D-Optimal EV & quenched & Measured bc & -0.0198 & 0.1069 & 0.1632 & -0.0988 & 0.955 & 1.279 & 1.456 & 0.797 \\
\hline SB5VS-05 & 34 & 418 & D-Optimal EV & $\mathrm{ccc}$ & targeted & -0.1185 & -0.0187 & -0.1119 & -0.2570 & 0.761 & 0.958 & 0.773 & 0.553 \\
\hline SB5VS-05 & 34 & 418 & D-Optimal EV & quenched & targeted & -0.1439 & -0.0556 & -0.1182 & -0.2892 & 0.718 & 0.880 & 0.762 & 0.514 \\
\hline $\begin{array}{l}\text { SB5VS-05 } \\
\end{array}$ & 34 & 418 & D-Optimal EV & $\mathrm{ccc}$ & Measured & -0.1251 & 0.0074 & -0.1084 & -0.2367 & 0.750 & 1.017 & 0.779 & 0.580 \\
\hline SB5VS-05 & 34 & 418 & D-Optimal EV & quenched & Measured & -0.1505 & -0.0295 & -0.1147 & -0.2689 & 0.707 & 0.934 & 0.768 & 0.538 \\
\hline SB5VS-05 & 34 & 418 & D-Optimal EV & $\mathrm{ccc}$ & Measured bc & -0.1195 & -0.0301 & -0.0973 & -0.2558 & 0.759 & 0.933 & 0.799 & 0.555 \\
\hline SB5VS-05 & 34 & 418 & D-Optimal EV & quenched & Measured bc & -0.1449 & -0.0670 & -0.1036 & -0.2880 & 0.716 & 0.857 & 0.788 & 0.515 \\
\hline SB5VS-06 & 34 & 418 & D-Optimal EV & $\mathrm{ccc}$ & targeted & -0.1443 & -0.0066 & -0.0664 & -0.2377 & 0.717 & 0.985 & 0.858 & 0.579 \\
\hline SB5VS-06 & 34 & 418 & D-Optimal EV & quenched & targeted & -0.1338 & -0.0067 & -0.0393 & -0.2335 & 0.735 & 0.985 & 0.914 & 0.584 \\
\hline SB5VS-06 & 34 & 418 & D-Optimal EV & $\mathrm{ccc}$ & Measured & -0.1806 & 0.0333 & -0.0694 & -0.2420 & 0.660 & 1.080 & 0.852 & 0.573 \\
\hline SB5VS-06 & 34 & 418 & D-Optimal EV & quenched & Measured & -0.1700 & 0.0333 & -0.0423 & -0.2378 & 0.676 & 1.080 & 0.907 & 0.578 \\
\hline SB5VS-06 & 34 & 418 & D-Optimal EV & $\mathrm{ccc}$ & Measured bc & -0.1992 & -0.0162 & -0.0643 & -0.2393 & 0.632 & 0.963 & 0.862 & 0.576 \\
\hline SB5VS-06 & 34 & 418 & D-Optimal EV & quenched & Measured bc & -0.1887 & -0.0163 & -0.0371 & -0.2352 & 0.648 & 0.963 & 0.918 & 0.582 \\
\hline SB5VS-07 & 34 & 418 & D-Optimal EV & $\mathrm{ccc}$ & targeted & -0.0220 & 0.0973 & 0.0885 & -0.1386 & 0.951 & 1.251 & 1.226 & 0.727 \\
\hline SB5VS-07 & 34 & 418 & D-Optimal EV & quenched & targeted & -0.0235 & 0.0919 & 0.1155 & -0.1236 & 0.947 & 1.236 & 1.305 & 0.752 \\
\hline SB5VS-07 & 34 & 418 & D-Optimal EV & $\mathrm{ccc}$ & Measured & -0.0490 & 0.1488 & 0.1129 & -0.1352 & 0.893 & 1.408 & 1.297 & 0.733 \\
\hline SB5VS-07 & 34 & 418 & D-Optimal EV & quenched & Measured & -0.0505 & 0.1434 & 0.1400 & -0.1202 & 0.890 & 1.391 & 1.380 & 0.758 \\
\hline SB5VS-07 & 34 & 418 & D-Optimal EV & $\mathrm{ccc}$ & Measured bc & -0.0678 & 0.0990 & 0.1223 & -0.1327 & 0.856 & 1.256 & 1.325 & 0.737 \\
\hline SB5VS-07 & 34 & 418 & D-Optimal EV & quenched & Measured bc & -0.0692 & 0.0936 & 0.1494 & -0.1177 & 0.853 & 1.241 & 1.411 & 0.763 \\
\hline
\end{tabular}


Table 6 cont. Normalized PCT Data

\begin{tabular}{|c|c|c|c|c|c|c|c|c|c|c|c|c|c|}
\hline Glass ID & WL(\%) & Frit & Sludge Case & $\begin{array}{c}\text { Heat } \\
\text { Treatment }\end{array}$ & Comp View & $\begin{array}{c}\log \mathrm{NL} \\
{[\mathrm{B}(\mathrm{g} / \mathrm{L})]} \\
\end{array}$ & $\begin{array}{c}\log \mathrm{NL} \\
{[\mathrm{Li}(\mathrm{g} / \mathrm{L})]}\end{array}$ & $\begin{array}{c}\log \mathrm{NL} \\
{[\mathrm{Na}(\mathrm{g} / \mathrm{L})]}\end{array}$ & $\begin{array}{c}\log \mathrm{NL} \\
{[\mathrm{Si}(\mathrm{g} / \mathrm{L})]} \\
\end{array}$ & $\begin{array}{c}\mathrm{NL} \\
\mathrm{B}(\mathrm{g} / \mathrm{L}) \\
\end{array}$ & $\begin{array}{c}\mathrm{NL} \\
\mathrm{Li}(\mathrm{g} / \mathrm{L})\end{array}$ & $\begin{array}{c}\mathrm{NL} \\
\mathrm{Na}(\mathrm{g} / \mathrm{L}) \\
\end{array}$ & $\begin{array}{c}\mathrm{NL} \\
\mathrm{Si}(\mathrm{g} / \mathrm{L}) \\
\end{array}$ \\
\hline SB5VS-08 & 34 & 418 & D-Optimal EV & $\mathrm{ccc}$ & targeted & -0.1502 & -0.0335 & -0.1270 & -0.2414 & 0.708 & 0.926 & 0.747 & 0.574 \\
\hline SB5VS-08 & 34 & 418 & D-Optimal EV & quenched & targeted & $\begin{array}{l}-0.1741 \\
\end{array}$ & $\begin{array}{l}-0.0597 \\
-1\end{array}$ & $\begin{array}{l}-0.1310 \\
\end{array}$ & $\begin{array}{l}-0.2537 \\
\end{array}$ & 0.670 & 0.871 & 0.740 & 0.558 \\
\hline SB5VS-08 & 34 & 418 & D-Optimal EV & $\mathrm{ccc}$ & Measured & $\begin{array}{l}-0.1456 \\
\end{array}$ & 0.0057 & -0.1207 & -0.2362 & 0.715 & 1.013 & 0.757 & 0.580 \\
\hline SB5VS-08 & 34 & 418 & D-Optimal EV & quenched & Measured & $\begin{array}{l}-0.1695 \\
\end{array}$ & $\begin{array}{l}-0.0205 \\
\end{array}$ & -0.1247 & $\begin{array}{l}-0.2485 \\
\end{array}$ & 0.677 & 0.954 & 0.750 & 0.564 \\
\hline SB5VS-08 & 34 & 418 & D-Optimal EV & $\mathrm{ccc}$ & Measured bc & -0.1621 & $\begin{array}{l}-0.0350 \\
\end{array}$ & -0.1113 & $\begin{array}{l}-0.2438 \\
\end{array}$ & 0.688 & 0.923 & 0.774 & 0.570 \\
\hline SB5VS-08 & 34 & 418 & D-Optimal EV & quenched & Measured bc & -0.1860 & -0.0612 & -0.1153 & -0.2561 & 0.652 & 0.868 & 0.767 & 0.554 \\
\hline SB5VS-09 & 34 & 418 & D-Optimal EV & ccc & targeted & $\begin{array}{l}-0.1598 \\
\end{array}$ & $\begin{array}{l}-0.0443 \\
\end{array}$ & -0.1126 & $\begin{array}{l}-0.2582 \\
\end{array}$ & 0.692 & 0.903 & 0.772 & 0.552 \\
\hline $\begin{array}{l}\text { SB5VS-09 } \\
\end{array}$ & 34 & 418 & D-Optimal EV & quenched & targeted & $\begin{array}{l}-0.1466 \\
\end{array}$ & $\begin{array}{l}-0.0403 \\
\end{array}$ & -0.0774 & -0.2476 & 0.713 & 0.911 & 0.837 & 0.565 \\
\hline SB5VS-09 & 34 & 418 & D-Optimal EV & ccc & Measured & $\begin{array}{l}-0.1755 \\
\end{array}$ & $\begin{array}{l}-0.0041 \\
\end{array}$ & -0.0902 & -0.2534 & 0.668 & 0.991 & 0.812 & 0.558 \\
\hline SB5VS-09 & 34 & 418 & D-Optimal EV & quenched & Measured & $\begin{array}{l}-0.1623 \\
\end{array}$ & $\begin{array}{l}-0.0002 \\
\end{array}$ & -0.0551 & $\begin{array}{l}-0.2429 \\
\end{array}$ & 0.688 & 1.000 & 0.881 & 0.572 \\
\hline $\begin{array}{l}\text { SB5VS-09 } \\
\end{array}$ & 34 & 418 & D-Optimal EV & $\mathrm{ccc}$ & Measured bc & $\begin{array}{l}-0.1922 \\
\end{array}$ & $\begin{array}{l}-0.0449 \\
\end{array}$ & -0.0851 & $\begin{array}{l}-0.2611 \\
\end{array}$ & 0.642 & 0.902 & 0.822 & 0.548 \\
\hline SB5VS-09 & 34 & 418 & D-Optimal EV & quenched & Measured bc & $\begin{array}{l}-0.1790 \\
\end{array}$ & $\begin{array}{l}-0.0409 \\
-\end{array}$ & $\begin{array}{l}-0.0499 \\
\end{array}$ & $\begin{array}{l}-0.2505 \\
\end{array}$ & 0.662 & 0.910 & 0.891 & 0.562 \\
\hline SB5VS-10 & 34 & 418 & D-Optimal EV & ccc & targeted & $\begin{array}{l}-0.1907 \\
\end{array}$ & $\begin{array}{l}-0.0611 \\
-0.0\end{array}$ & -0.1460 & $\begin{array}{l}-0.2564 \\
\end{array}$ & 0.645 & 0.869 & 0.715 & 0.554 \\
\hline SB5VS-10 & 34 & 418 & D-Optimal EV & quenched & targeted & $\begin{array}{l}-0.1885 \\
\end{array}$ & $\begin{array}{l}-0.0521 \\
\end{array}$ & -0.1212 & $\begin{array}{l}-0.2692 \\
\end{array}$ & 0.648 & 0.887 & 0.756 & 0.538 \\
\hline SB5VS-10 & 34 & 418 & D-Optimal EV & ccc & Measured & $\begin{array}{l}-0.1868 \\
\end{array}$ & -0.0126 & -0.1414 & $\begin{array}{c}-0.2511 \\
\end{array}$ & 0.650 & 0.971 & 0.722 & 0.561 \\
\hline SB5VS-10 & 34 & 418 & D-Optimal EV & quenched & Measured & $\begin{array}{l}-0.1846 \\
\end{array}$ & -0.0036 & -0.1166 & -0.2640 & 0.654 & 0.992 & 0.764 & 0.545 \\
\hline SB5VS-10 & 34 & 418 & D-Optimal EV & $\mathrm{ccc}$ & Measured bc & -0.2056 & -0.0623 & -0.1320 & -0.2486 & 0.623 & 0.866 & 0.738 & 0.564 \\
\hline SB5VS-10 & 34 & 418 & D-Optimal EV & quenched & Measured bc & $\begin{array}{l}-0.2034 \\
\end{array}$ & $\begin{array}{l}-0.0533 \\
\end{array}$ & -0.1072 & -0.2615 & 0.626 & 0.885 & 0.781 & 0.548 \\
\hline SB5VS-11 & 34 & 418 & D-Optimal EV & ccc & targeted & $\begin{array}{l}-0.0753 \\
\end{array}$ & 0.0241 & -0.0439 & $\begin{array}{l}-0.1911 \\
\end{array}$ & 0.841 & 1.057 & 0.904 & 0.644 \\
\hline SB5VS-11 & 34 & 418 & D-Optimal EV & quenched & targeted & $\begin{array}{l}-0.0845 \\
\end{array}$ & 0.0117 & -0.0211 & $\begin{array}{l}-0.1971 \\
\end{array}$ & 0.823 & 1.027 & 0.952 & 0.635 \\
\hline SB5VS-11 & 34 & 418 & D-Optimal EV & $\mathrm{ccc}$ & Measured & $\begin{array}{l}-0.0766 \\
\end{array}$ & 0.0388 & -0.0452 & -0.2045 & 0.838 & 1.094 & 0.901 & 0.624 \\
\hline SB5VS-11 & 34 & 418 & D-Optimal EV & quenched & Measured & $\begin{array}{l}-0.0858 \\
\end{array}$ & 0.0264 & -0.0224 & $\begin{array}{l}-0.2105 \\
\end{array}$ & 0.821 & 1.063 & 0.950 & 0.616 \\
\hline SB5VS-11 & 34 & 418 & D-Optimal EV & $\mathrm{ccc}$ & Measured bc & $\begin{array}{l}-0.0932 \\
-\end{array}$ & $\begin{array}{l}-0.0018 \\
-0.0\end{array}$ & -0.0400 & -0.2120 & 0.807 & 0.996 & 0.912 & 0.614 \\
\hline SB5VS-11 & 34 & 418 & D-Optimal EV & quenched & Measured bc & $\begin{array}{l}-0.1024 \\
\end{array}$ & $\begin{array}{l}-0.0142 \\
\end{array}$ & -0.0172 & $\begin{array}{l}-0.2180 \\
\end{array}$ & 0.790 & 0.968 & 0.961 & 0.605 \\
\hline SB5VS-12 & 34 & 418 & D-Optimal EV & ccc & targeted & $\begin{array}{l}-0.0352 \\
\end{array}$ & 0.1071 & 0.0615 & -0.1065 & 0.922 & 1.280 & 1.152 & 0.782 \\
\hline SB5VS-12 & 34 & 418 & D-Optimal EV & quenched & targeted & $\begin{array}{c}-0.0360 \\
\end{array}$ & 0.0676 & 0.0914 & $\begin{array}{l}-0.0760 \\
\end{array}$ & 0.920 & 1.169 & 1.234 & 0.839 \\
\hline SB5VS-12 & 34 & 418 & D-Optimal EV & ccc & Measured & $\begin{array}{l}-0.0386 \\
\end{array}$ & 0.1313 & 0.0574 & $\begin{array}{l}-0.0985 \\
\end{array}$ & 0.915 & 1.353 & 1.141 & 0.797 \\
\hline SB5VS-12 & 34 & 418 & D-Optimal EV & quenched & Measured & $\begin{array}{l}-0.0394 \\
\end{array}$ & 0.0918 & 0.0873 & $\begin{array}{l}-0.0680 \\
\end{array}$ & 0.913 & 1.235 & 1.223 & 0.855 \\
\hline SB5VS-12 & 34 & 418 & D-Optimal EV & $\mathrm{ccc}$ & Measured bc & $\begin{array}{l}-0.0551 \\
\end{array}$ & 0.0906 & 0.0625 & $\begin{array}{l}-0.1061 \\
\end{array}$ & 0.881 & 1.232 & 1.155 & 0.783 \\
\hline SB5VS-12 & 34 & 418 & D-Optimal EV & quenched & Measured bc & $\begin{array}{l}-0.0559 \\
\end{array}$ & 0.0512 & 0.0924 & $\begin{array}{l}-0.0756 \\
\end{array}$ & 0.879 & 1.125 & 1.237 & 0.840 \\
\hline SB5VS-13 & 34 & 418 & SB5 Centroid & ccc & targeted & $\begin{array}{l}-0.1153 \\
\end{array}$ & $\begin{array}{l}-0.0027 \\
\end{array}$ & -0.0537 & -0.2230 & 0.767 & 0.994 & 0.884 & 0.598 \\
\hline SB5VS-13 & 34 & 418 & SB5 Centroid & quenched & targeted & -0.1118 & -0.0127 & -0.0341 & -0.2097 & 0.773 & 0.971 & 0.925 & 0.617 \\
\hline SB5VS-13 & 34 & 418 & SB5 Centroid & $\mathrm{ccc}$ & Measured & $\begin{array}{ll}-0.1147 \\
\end{array}$ & 0.0263 & -0.0464 & $\begin{array}{c}-0.2301 \\
\end{array}$ & 0.768 & 1.062 & 0.899 & 0.589 \\
\hline SB5VS-13 & 34 & 418 & SB5 Centroid & quenched & Measured & -0.1112 & 0.0163 & -0.0268 & -0.2168 & 0.774 & 1.038 & 0.940 & 0.607 \\
\hline SB5VS-13 & 34 & 418 & SB5 Centroid & $\mathrm{ccc}$ & Measured bc & -0.1312 & -0.0143 & -0.0370 & -0.2376 & 0.739 & 0.968 & 0.918 & 0.579 \\
\hline SB5VS-13 & 34 & 418 & SB5 Centroid & quenched & Measured bc & -0.1277 & -0.0243 & -0.0174 & -0.2243 & 0.745 & 0.946 & 0.961 & 0.597 \\
\hline SB5VS-14 & 34 & 418 & BS-07 (C40/C51 25/75 w 3\% SO) & $\mathrm{ccc}$ & targeted & -0.1419 & -0.0475 & -0.0547 & -0.2378 & 0.721 & 0.896 & 0.882 & 0.578 \\
\hline SB5VS-14 & 34 & 418 & $\mathrm{BS}-07$ (C40/C51 25/75 w 3\% SO) & quenched & targeted & $\begin{array}{c}-0.1349 \\
\end{array}$ & -0.0672 & -0.0572 & -0.2490 & 0.733 & 0.857 & 0.877 & 0.564 \\
\hline SB5VS-14 & 34 & 418 & BS-07 (C40/C51 25/75 w 3\% SO) & $\mathrm{ccc}$ & Measured & $\begin{array}{c}-0.1601 \\
\end{array}$ & $\begin{array}{c}-0.0149 \\
\end{array}$ & -0.0515 & -0.2138 & 0.692 & 0.966 & 0.888 & 0.611 \\
\hline SB5VS-14 & 34 & 418 & BS-07 (C40/C51 25/75 w 3\% SO) & quenched & Measured & \begin{tabular}{c|c|c|}
-0.1531 \\
\end{tabular} & -0.0345 & -0.0540 & -0.2250 & 0.703 & 0.924 & 0.883 & 0.596 \\
\hline SB5VS-14 & 34 & 418 & $\mathrm{BS}-07(\mathrm{C} 40 / \mathrm{C} 5125 / 75$ w $3 \% \mathrm{SO})$ & $\mathrm{ccc}$ & Measured bc & -0.1545 & $\begin{array}{c}-0.0524 \\
\end{array}$ & -0.0404 & -0.2330 & 0.701 & 0.886 & 0.911 & 0.585 \\
\hline SB5VS-14 & 34 & 418 & BS-07 (C40/C51 25/75 w 3\% SO) & quenched & Measured bc & $\begin{array}{l}-0.1475 \\
\end{array}$ & $\begin{array}{l}-0.0721 \\
-070\end{array}$ & -0.0429 & \begin{tabular}{c|c|c|c|}
-0.2442 \\
\end{tabular} & 0.712 & 0.847 & 0.906 & 0.570 \\
\hline SB5VS-15 & 34 & 418 & BS-15 (C40/C51 25/75 w 3\% ARP) & $\mathrm{ccc}$ & targeted & -0.1490 & -0.0561 & -0.0593 & -0.2457 & 0.710 & 0.879 & 0.872 & 0.568 \\
\hline SB5VS-15 & 34 & 418 & BS-15 (C40/C51 25/75 w 3\% ARP) & quenched & targeted & -0.1515 & $\begin{array}{l}-0.0629 \\
\end{array}$ & -0.0378 & $\begin{array}{c}-0.2443 \\
\end{array}$ & 0.706 & 0.865 & 0.917 & 0.570 \\
\hline SB5VS-15 & 34 & 418 & BS-15 (C40/C51 25/75 w 3\% ARP) & $\mathrm{ccc}$ & Measured & -0.1081 & -0.0177 & -0.0566 & -0.2481 & 0.780 & 0.960 & 0.878 & 0.565 \\
\hline SB5VS-15 & 34 & 418 & BS-15 (C40/C51 25/75 w 3\% ARP) & quenched & Measured & -0.1106 & -0.0245 & -0.0351 & $\begin{array}{c}-0.2468 \\
\end{array}$ & 0.775 & 0.945 & 0.922 & 0.567 \\
\hline SB5VS-15 & 34 & 418 & BS-15 (C40/C51 25/75 w 3\% ARP) & $\mathrm{ccc}$ & Measured bc & -0.1267 & -0.0673 & -0.0472 & -0.2456 & 0.747 & 0.856 & 0.897 & 0.568 \\
\hline SB5VS-15 & 34 & 418 & BS-15 (C40/C51 25/75 w 3\% ARP) & quenched & Measured bc & -0.1292 & $\begin{array}{c}-0.0741 \\
\end{array}$ & -0.0258 & -0.2442 & 0.743 & 0.843 & 0.942 & 0.570 \\
\hline
\end{tabular}


Table 6 cont. Normalized PCT Data

\begin{tabular}{|c|c|c|c|c|c|c|c|c|c|c|c|c|c|}
\hline Glass ID & $\mathrm{WL}(\%)$ & Frit & Sludge Case & $\begin{array}{c}\text { Heat } \\
\text { Treatment }\end{array}$ & Comp View & $\begin{array}{c}\log \mathrm{NL} \\
{[\mathrm{B}(\mathrm{g} / \mathrm{L})]}\end{array}$ & $\begin{array}{c}\log \mathrm{NL} \\
{[\mathrm{Li}(\mathrm{g} / \mathrm{L})]}\end{array}$ & $\begin{array}{c}\log \mathrm{NL} \\
{[\mathrm{Na}(\mathrm{g} / \mathrm{L})]}\end{array}$ & $\begin{array}{c}\log \mathrm{NL} \\
{[\mathrm{Si}(\mathrm{g} / \mathrm{L})]}\end{array}$ & $\begin{array}{c}\mathrm{NL} \\
\mathrm{B}(\mathrm{g} / \mathrm{L})\end{array}$ & $\begin{array}{c}\mathrm{NL} \\
\mathrm{Li}(\mathrm{g} / \mathrm{L})\end{array}$ & $\begin{array}{c}\mathrm{NL} \\
\mathrm{Na}(\mathrm{g} / \mathrm{L})\end{array}$ & $\begin{array}{c}\mathrm{NL} \\
\mathrm{Si}(\mathrm{g} / \mathrm{L})\end{array}$ \\
\hline SB5VS-16 & 31 & 418 & BS-07 (C40/C51 25/75 w 3\% SO) & $\mathrm{ccc}$ & targeted & -0.1498 & -0.0258 & -0.0530 & -0.2328 & 0.708 & 0.942 & 0.885 & 0.585 \\
\hline SB5VS-16 & 31 & 418 & BS-07 (C40/C51 25/75 w 3\% SO) & quenched & targeted & -0.1549 & -0.0488 & -0.0547 & -0.2388 & 0.700 & 0.894 & 0.882 & 0.577 \\
\hline SB5VS-16 & 31 & 418 & BS-07 (C40/C51 25/75 w 3\% SO) & $\mathrm{ccc}$ & Measured & -0.1582 & 0.0200 & -0.0512 & -0.2296 & 0.695 & 1.047 & 0.889 & 0.589 \\
\hline SB5VS-16 & 31 & 418 & BS-07 (C40/C51 25/75 w 3\% SO) & quenched & Measured & -0.1632 & -0.0030 & -0.0529 & -0.2355 & 0.687 & 0.993 & 0.885 & 0.581 \\
\hline SB5VS-16 & 31 & 418 & BS-07 (C40/C51 25/75 w 3\% SO) & $\mathrm{ccc}$ & Measured bc & -0.1769 & -0.0295 & -0.0418 & -0.2269 & 0.665 & 0.934 & 0.908 & 0.593 \\
\hline SB5VS-16 & 31 & 418 & BS-07 (C40/C51 25/75 w 3\% SO) & quenched & Measured bc & -0.1819 & -0.0524 & -0.0435 & -0.2328 & 0.658 & 0.886 & 0.905 & 0.585 \\
\hline SB5VS-17 & 37 & 418 & BS-07 (C40/C51 25/75 w 3\% SO) & $\mathrm{ccc}$ & targeted & -0.0964 & -0.0304 & -0.0458 & -0.2281 & 0.801 & 0.932 & 0.900 & 0.591 \\
\hline SB5VS-17 & 37 & 418 & BS-07 (C40/C51 25/75 w 3\% SO) & quenched & targeted & -0.1345 & -0.0687 & -0.0480 & -0.2683 & 0.734 & 0.854 & 0.895 & 0.539 \\
\hline $\begin{array}{l}\text { SB5VS-17 } \\
\end{array}$ & 37 & 418 & BS-07 (C40/C51 25/75 w 3\% SO) & $\mathrm{ccc}$ & Measured & -0.1187 & -0.0059 & -0.0268 & -0.2196 & 0.761 & 0.987 & 0.940 & 0.603 \\
\hline SB5VS-17 & 37 & 418 & BS-07 (C40/C51 25/75 w 3\% SO) & quenched & Measured & -0.1567 & -0.0442 & -0.0290 & -0.2598 & 0.697 & 0.903 & 0.935 & 0.550 \\
\hline SB5VS-17 & 37 & 418 & BS-07 (C40/C51 25/75 w 3\% SO) & $\mathrm{ccc}$ & Measured bc & -0.1131 & -0.0434 & -0.0157 & -0.2387 & 0.771 & 0.905 & 0.964 & 0.577 \\
\hline SB5VS-17 & 37 & 418 & BS-07 (C40/C51 25/75 w 3\% SO) & quenched & Measured bc & -0.1512 & -0.0817 & -0.0179 & -0.2789 & 0.706 & 0.829 & 0.960 & 0.526 \\
\hline $\begin{array}{l}\text { SB5VS-18 } \\
\end{array}$ & 25 & 418 & BS-15 (C40/C51 25/75 w 3\% ARP) & $\mathrm{ccc}$ & targeted & -0.1323 & -0.0325 & -0.0816 & -0.1924 & 0.737 & 0.928 & 0.829 & 0.642 \\
\hline SB5VS-18 & 25 & 418 & BS-15 (C40/C51 25/75 w 3\% ARP) & quenched & targeted & -0.1454 & -0.0220 & -0.0604 & -0.2236 & 0.715 & 0.951 & 0.870 & 0.598 \\
\hline $\begin{array}{l}\text { SB5VS-18 } \\
\end{array}$ & 25 & 418 & BS-15 (C40/C51 25/75 w 3\% ARP) & $\mathrm{ccc}$ & Measured & -0.1321 & -0.0073 & -0.0581 & -0.1900 & 0.738 & 0.983 & 0.875 & 0.646 \\
\hline $\begin{array}{l}\text { SB5VS-18 } \\
\end{array}$ & 25 & 418 & BS-15 (C40/C51 25/75 w 3\% ARP) & quenched & Measured & -0.1452 & 0.0032 & -0.0369 & -0.2213 & 0.716 & 1.007 & 0.919 & 0.601 \\
\hline $\begin{array}{l}\text { SB5VS-18 } \\
\end{array}$ & 25 & 418 & BS-15 (C40/C51 25/75 w 3\% ARP) & $\mathrm{ccc}$ & Measured bc & -0.1486 & -0.0480 & -0.0487 & -0.1977 & 0.710 & 0.895 & 0.894 & 0.634 \\
\hline SB5VS-18 & 25 & 418 & BS-15 (C40/C51 25/75 w 3\% ARP) & quenched & Measured bc & -0.1618 & -0.0375 & -0.0275 & -0.2289 & 0.689 & 0.917 & 0.939 & 0.590 \\
\hline SB5VS-19 & 28 & 418 & BS-15 (C40/C51 25/75 w 3\% ARP) & $\mathrm{ccc}$ & targeted & -0.1517 & -0.0212 & -0.0614 & -0.2012 & 0.705 & 0.952 & 0.868 & 0.629 \\
\hline $\begin{array}{l}\text { SB5VS-19 } \\
\end{array}$ & 28 & 418 & BS-15 (C40/C51 25/75 w 3\% ARP) & quenched & targeted & -0.1335 & -0.0045 & -0.0189 & -0.2009 & 0.735 & 0.990 & 0.957 & 0.630 \\
\hline $\begin{array}{l}\text { SB5VS-19 } \\
\end{array}$ & 28 & 418 & BS-15 (C40/C51 25/75 w 3\% ARP) & $\mathrm{ccc}$ & Measured & -0.1390 & -0.0054 & -0.0619 & $\begin{array}{ll}-0.1968 \\
\end{array}$ & 0.726 & 0.988 & 0.867 & 0.636 \\
\hline $\begin{array}{l}\text { SB5VS-19 } \\
\end{array}$ & 28 & 418 & BS-15 (C40/C51 25/75 w 3\% ARP) & quenched & Measured & -0.1208 & 0.0113 & -0.0194 & -0.1965 & 0.757 & 1.026 & 0.956 & 0.636 \\
\hline $\begin{array}{l}\text { SB5VS-19 } \\
\end{array}$ & 28 & 418 & BS-15 (C40/C51 25/75 w 3\% ARP) & $\mathrm{ccc}$ & Measured bc & -0.1557 & -0.0461 & -0.0568 & -0.2044 & 0.699 & 0.899 & 0.877 & 0.625 \\
\hline $\begin{array}{l}\text { SB5VS-19 } \\
\end{array}$ & 28 & 418 & BS-15 (C40/C51 25/75 w 3\% ARP) & quenched & Measured bc & -0.1374 & -0.0294 & -0.0143 & -0.2040 & 0.729 & 0.935 & 0.968 & 0.625 \\
\hline SB5VS-20 & 31 & 418 & BS-15 (C40/C51 25/75 w 3\% ARP) & $\mathrm{ccc}$ & targeted & -0.1185 & -0.0289 & -0.0606 & -0.2102 & 0.761 & 0.936 & 0.870 & 0.616 \\
\hline $\begin{array}{l}\text { SB5VS-20 } \\
\end{array}$ & 31 & 418 & BS-15 (C40/C51 25/75 w 3\% ARP) & quenched & targeted & -0.1313 & -0.0340 & -0.0384 & -0.2101 & 0.739 & 0.925 & 0.915 & 0.616 \\
\hline SB5VS-20 & 31 & 418 & BS-15 (C40/C51 25/75 w 3\% ARP) & $\mathrm{ccc}$ & Measured & -0.1244 & -0.0011 & -0.0487 & -0.1836 & 0.751 & 0.997 & 0.894 & 0.655 \\
\hline SB5VS-20 & 31 & 418 & BS-15 (C40/C51 25/75 w 3\% ARP) & quenched & Measured & -0.1371 & -0.0062 & -0.0265 & -0.1835 & 0.729 & 0.986 & 0.941 & 0.655 \\
\hline $\begin{array}{l}\text { SB5VS-20 } \\
\end{array}$ & 31 & 418 & BS-15 (C40/C51 25/75 w 3\% ARP) & $\mathrm{ccc}$ & Measured bc & -0.1188 & -0.0387 & -0.0376 & -0.2027 & 0.761 & 0.915 & 0.917 & 0.627 \\
\hline SB5VS-20 & 31 & 418 & BS-15 (C40/C51 25/75 w 3\% ARP) & quenched & Measured bc & -0.1315 & -0.0438 & -0.0154 & -0.2027 & 0.739 & 0.904 & 0.965 & 0.627 \\
\hline $\begin{array}{l}\text { SB5VS-21 } \\
\end{array}$ & 37 & 418 & BS-15 (C40/C51 25/75 w 3\% ARP) & $\mathrm{ccc}$ & targeted & -0.0863 & -0.0235 & -0.0176 & -0.2101 & 0.820 & 0.947 & 0.960 & 0.617 \\
\hline SB5VS-21 & 37 & 418 & BS-15 (C40/C51 25/75 w 3\% ARP) & quenched & targeted & -0.0827 & -0.0443 & -0.0058 & -0.2089 & 0.827 & 0.903 & 0.987 & 0.618 \\
\hline $\begin{array}{l}\text { SB5VS-21 } \\
\end{array}$ & 37 & 418 & BS-15 (C40/C51 25/75 w 3\% ARP) & $\mathrm{ccc}$ & Measured & -0.1112 & 0.0109 & 0.0001 & -0.1999 & 0.774 & 1.026 & 1.000 & 0.631 \\
\hline SB5VS-21 & 37 & 418 & BS-15 (C40/C51 25/75 w 3\% ARP) & quenched & Measured & -0.1076 & -0.0099 & 0.0119 & -0.1987 & 0.781 & 0.977 & 1.028 & 0.633 \\
\hline $\begin{array}{l}\text { SB5VS-21 } \\
\end{array}$ & 37 & 418 & BS-15 (C40/C51 25/75 w 3\% ARP) & $\mathrm{ccc}$ & Measured bc & -0.1056 & -0.0266 & 0.0112 & -0.2189 & 0.784 & 0.941 & 1.026 & 0.604 \\
\hline $\begin{array}{l}\text { SB5VS-21 } \\
\end{array}$ & 37 & 418 & BS-15 (C40/C51 25/75 w 3\% ARP) & quenched & Measured bc & -0.1020 & -0.0474 & 0.0230 & -0.2178 & 0.791 & 0.897 & 1.054 & 0.606 \\
\hline
\end{tabular}




\subsection{References}

1. K.M. Fox, T.B. Edwards, and D.K. Peeler, "Recommended Frit Composition for Initial Sludge Batch 5 Processing at the Defense Waste Processing Facility," Savannah River National Laboratory, Aiken, SC, WSRC-STI-2008-00338, Rev. 0, 2008.

2. B.H. Culbertson, "Sludge Batch 5 Glass Variability Studies," Defense Waste Processing Facility, Aiken, SC, Technical Task Request HLW-DWPF-TTR-2007-0009, Rev. 0, 2006.

3. D.K. Peeler, "Sludge Batch 5 Glass Variability Studies," Savannah River National Laboratory, Aiken, SC, WSRC-STI-2006-00320, Rev. 0, 2007.

4. $\quad \mathrm{JMP}^{\mathrm{TM}}$ Version 6.0.3, SAS Institute Inc., Cary, NC, 2005.

5. B.R. Pickenheim, J.M. Pareizs, C.J. Bannochie, H.M. Ajo, and S.H. Reboul, "Data from the SRNL Shielded Cells Demonstration of the DWPF Srat Using SB5 Tank 51 Qualification Sample (SC-6)," Savannah River National Laboratory, Aiken, SC, SRNL-PSE-2008-00157, 2008.

6. C.C. Herman, T.B. Edwards, D.R. Best, D.M. Marsh, and R.J. Workman, "Reduction of Constraints: Phase 2 Experimental Assessment for Sludge-Only Processing," Savannah River National Laboratory, Aiken, SC, WSRC-TR-2002-00482 Rev. 0, 2002.

7. F.C. Raszewski and M.E. Stone, "Experimental Testing to Support Additional SB4 Tank 40 Decants (TT\&QAP)," Savannah River National Laboratory, Aiken, SC, WSRC-STI-2008-00254, Rev. 0, 2008.

8. S.L. Marra and C.M. Jantzen, "Characterization of Projected DWPF Glass Heat Treated to Simulate Canister Centerline Cooling," Westinghouse Savannah River Company, Aiken, SC, WSRC-TR-92-142, Rev. 1, 1993.

9. T.B. Edwards, "An Analytical Plan for Measuring the Chemical Compositions of SB5 Variability Study Glasses," Savannah River National Laboratory, Aiken, SC, SRNL-SCS-2008-00071, 2008.

10. "Standard Test Methods for Determining Chemical Durability of Nuclear, Hazardous, and Mixed Waste Glasses and Multiphase Glass Ceramics: The Product Consistency Test (PCT)," ASTM International, West Conshohocken, PA, ASTM C 1285-02, 2002.

11. T.B. Edwards, "An Analytical Plan for Measuring the First Set of PCT Solutions for the SB5 VS Glasses," Savannah River National Laboratory, Aiken, SC, SRNL-SCS-2008-00072, 2008.

12. T.B. Edwards, "An Analytical Plan for Measuring the Second Set of PCT Solutions for the SB5 VS Glasses," Savannah River National Laboratory, Aiken, SC, SRNL-SCS-2008-00073, 2008.

13. F.C. Raszewski, T.B. Edwards, and D.K. Peeler, "A Review of the Chemical Compositional and PCT Measurements for the SB5 Variability Study Glasses," Savannah River National Laboratory, Aiken, SC, SRNL-L3100-2008-00012, 2008. 


\section{Distribution:}

C.J. Bannochie, 773-42A

A.B. Barnes, 999-W

D.R. Best, 786-1A

A.L. Billings, 999-W

J.M. Bricker, 704-27S

D.B. Burns, 786-5A

B.A. Davis, 704-27S

T.B. Edwards, 999-W

H.H. Elder, 766-H

T.L. Fellinger, 704-26S

K.M. Fox, 999-W

B.J. Giddings, 786-5A

J.M. Gillam, 766-H

J.C. Griffin, 773-A

B.A. Hamm, 766-H

C.C. Herman, 999-W

M.T. Keefer, 766-H

J.E. Marra, 773-A

R.T. McNew, 704-27S

T.A. Nance, $773-42 \mathrm{~A}$

J.D. Newell, 999-W

J.E. Occhipinti, 704-S

D.K. Peeler, 999-W

F.C. Raszewski, 999-W

J.W. Ray, 704-S

A.R. Shafer, 704-27S

H.B. Shah, 766-H

M.E. Stone, 999-W

M.F. Williams, 999-1W 